\title{
Antimicrobial and genetic strategies to defend kiwifruit against the bacterial pathogen Pseudomonas syringae pathovar actinidiae (Psa).
}

A thesis submitted to the Victoria University of Wellington

$$
\text { by }
$$

Mathew Ambrose Storey

In partial fulfilment of the requirements for the degree

Masters by thesis in Biotechnology

\section{4}

TE WHARE WĀNANGA O TE ŪPOKO O TE IKA A MĀUI

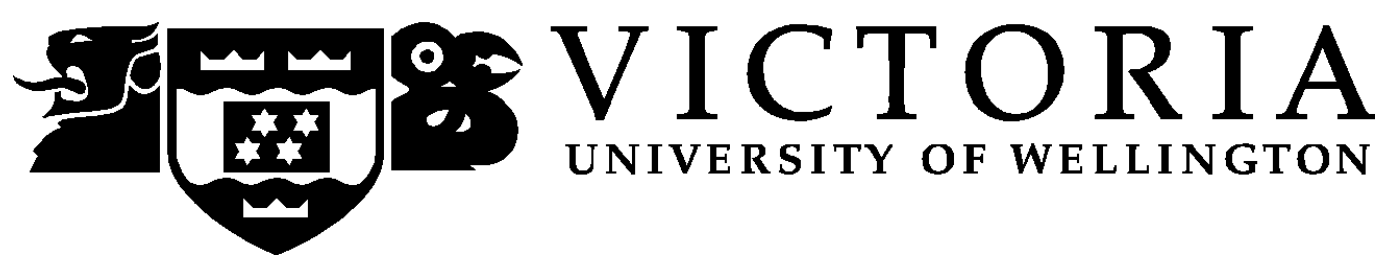




\title{
Antimicrobial and genetic strategies to defend kiwifruit against the bacterial pathogen Pseudomonas syringae pathovar actinidiae (Psa).
}

\author{
Matt Storey
}

\begin{abstract}
A highly virulent strain of the bacterium Pseudomonas syringae pv. actinidiae (Psa-V), the causative agent of bacterial canker of kiwifruit, is threatening the $\$ 1.5$ billion New Zealand kiwifruit industry. A strain of Psa-V was first identified in Italy in 2008 and related strain with a similar level of virulence arrived in New Zealand in November 2010. Since then it has been spreading rapidly throughout the country with devastating effects. Currently there is no effective treatment for growers to control Psa-V in their orchards and the potential impact of Psa-V on the New Zealand kiwifruit industry and growers is catastrophic. As part of a collaboration between Seeka Kiwifruit Industries, EastPack NZ, and a group of scientists nationwide (Taskforce Green) this work designed and implemented laboratory tests to quantify the effect of candidate antimicrobial sprays. Novel formulations with strong antimicrobial properties, including silver nanoparticles were also tested. This work was complemented by an investigation into the antibiotic resistance potential of Psa-V. A spontaneous streptomycin resistant mutant of Psa-V was generated and the molecular mechanism of resistance was elucidated. Further, gene knockout strategies aimed at facilitating the study of Psa-V virulence genes and ultimately developing a potential biocontrol agent were tested. Overall, this work together with several recent advances in the field should help advise the kiwifruit industry on best practice around the use of anti-Psa-V agents, and may make it possible to facilitate the generation and testing of candidate biocontrol agents in the near future.
\end{abstract}




\section{Dedication.}

This thesis work is dedicated to Stephen Cook Jr. 


\section{Acknowledgements.}

The work in this thesis was made possible by the kind and persistent support that has come from many generous institutions and devoted individuals throughout the course of this project. I extend my utmost, heartfelt gratitude and respect to the good nature of all the people who have graciously contributed.

I would like to especially thank the following -

Dr. Dave Ackerley (Supervisor),

DFA lab group (Victoria university Wellington),

Michael Franks (Funding contributions),

Dr. Matt Templeton (Genome data),

Dr. Honour McCann (Expertise),

Dr Elena Colombi (Expertise),

Dr Stanley Bellgard (Landcare Research),

Prof. Iain Lamont (University of Otago),

Dr. Russell Poulter (University of Otago),

Shalinee Dowlut (Verified lab services). 


\section{Contents}

1 Introduction $\quad 1$

1.1 The host - Actinidia spp. - an industry, an identity, a monoculture. . . . . . 1

1.1.1 Buds of industry: Cultivar development and global dispersal . . . . . 1

1.1.2 The genetic bottleneck . . . . . . . . . . . . . . . . 2

1.2 The pathogen - Pseudomonas syringae pv. actinidiae: the causative agent of bacterial canker of kiwifruit . . . . . . . . . . . . . . . . 2

1.2.1 P. syringae - classification and description . . . . . . . . . . . 2

$1.2 .2 \quad$ P. syringae: lifecycle. . . . . . . . . . . . . . 3

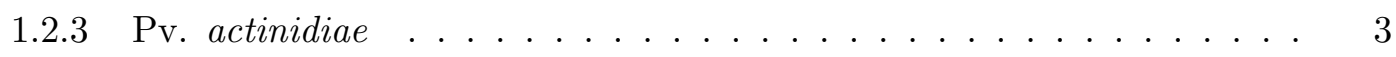

1.2 .4 First isolation . . . . . . . . . . . . . . . . . 4

1.2 .5 Psa in New Zealand . . . . . . . . . . . . . . . . . 4

1.2.6 Effects of Psa-V infection . . . . . . . . . . . . . 7

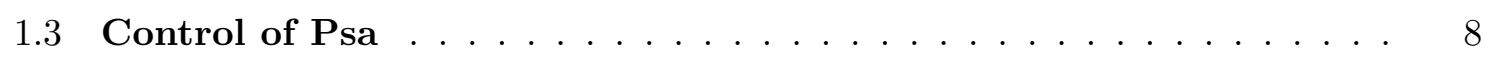

1.3.1 Previously trialled and current control methods . . . . . . . . . . . 8

1.4 Proposed alternative control measures . . . . . . . . . . . . . . 10

1.4.1 New bactericidal compounds effective against Psa-V . . . . . . . 10

1.4.2 Biological control by antagonistic micro-organisms . . . . . . . . . . . 10

1.4.3 Possible biocontrol of Psa-V . . . . . . . . . . . . . . . . . 11

1.5 Plant immunity and pathogen-host interactions . . . . . . . . . . 12

1.5.1 Basal plant immunity . . . . . . . . . . . . . . . 12

1.5.2 Basal plant immune response suppression by pathogen secreted effectors 15

1.5.3 Plant resistance to effector mediated attack . . . . . . . . . . . 16

1.5.4 Summary / closing remarks on immunity . . . . . . . . . . . . . . . . . 17

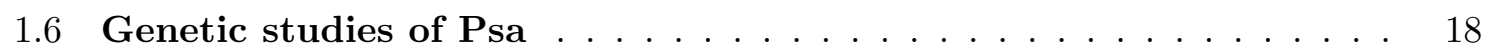

1.6 .1 Genetic studies . . . . . . . . . . . . . . . . . . . . . . . . . . . . 18

1.6.2 Psa population diversity . . . . . . . . . . . . . . . . . 19

1.6 .3 Genome features of Psa . . . . . . . . . . . . . . . 19

1.6 .4 Origins of $\mathrm{Psa}-\mathrm{V} \ldots \ldots \ldots \ldots 20 \ldots \ldots$

1.6.5 Integrative conjugative elements - ICEs . . . . . . . . . . . . . . . . 21

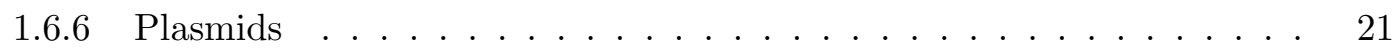

1.6 .7 Toxin production . . . . . . . . . . . . . . . . . . . . . . . 22

1.6 .8 Unique effectors . . . . . . . . . . . . . . . . 22

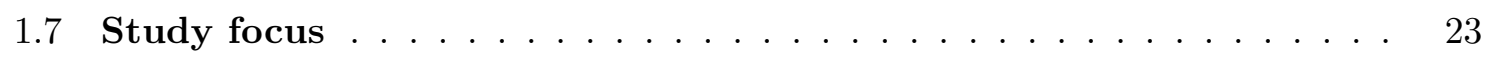

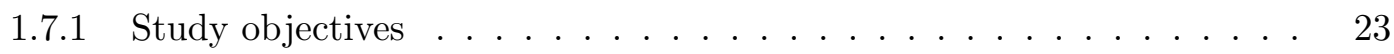


1.7 .2 Considerations . . . . . . . . . . . . . . . . . 23

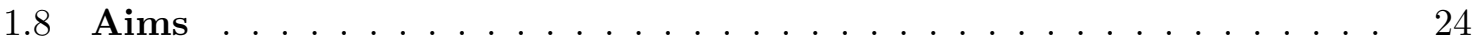

1.8.1 Assay to rapidly test a range compounds . . . . . . . . . . . . . . . . . . . . . . . 24

1.8.2 In-frame gene knockouts of Psa-V . . . . . . . . . . . . . . . 24

2 Materials and Methods $\quad 26$

2.1 Chemicals, media, enzymes and reagents supplies. . . . . . . . . . . 26

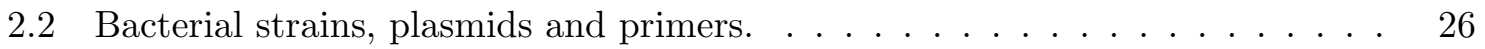

2.2 .1 Bacterial Strains . . . . . . . . . . . . . . . . . 26

2.2 .2 Plasmids . . . . . . . . . . . . . . . . . . 27

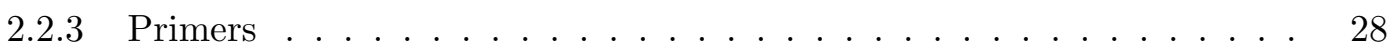

2.3 Bacterial growth and maintenance . . . . . . . . . . . . . 30

2.3.1 Bacterial growth media. . . . . . . . . . . . . . . 30

2.3.2 Media supplements . . . . . . . . . . . . . . . . . . . 31

2.3 .3 Bacterial growth conditions . . . . . . . . . . . . . . 32

2.4 Routine molecular biology . . . . . . . . . . . . . . . . . . . . 32

2.4 .1 PCR protocols . . . . . . . . . . . . . . . . 32

2.4.2 Standard BioMix $\operatorname{Red}^{\mathrm{TM}} \mathrm{PCR} \ldots \ldots \ldots \ldots . \ldots \ldots$

2.5 DNA extraction purification and manipulation. . . . . . . . . . 33

2.5.1 Tris Acetate-EDTA (TAE) agarose gel electrophoresis . . . . . . . . . 34

2.6 Overlap PCR for mutagenic fragment generation. . . . . . . . . . . . . . . . 35

2.7 Preparation and transformation of competent cells _ . . . . . . . . . . 37

2.7.1 Preparation and transformation of chemically competent E. coli . . . 37

2.7.2 Preparation and transformation of electrocompetent E. coli . . . . . . 38

2.7.3 Preparation and transformation of electrocompetent Pseudomonas. . . 39

2.7.4 Transformation by bacterial conjugation as a means of plasmid transfer into Pseudomonas species. . . . . . . . . . . . . . . . . . . . . . . . . . . . .

2.8 Screening to identify recombinant E. coli clones . . . . . . . . . . . . . . 40

2.8.1 Colony PCR . . . . . . . . . . . . . . . 40

2.8.2 Confirmation by restriction digest . . . . . . . . . . . . . . 40

2.9 In-frame deletion of genes from the chromosomes of Psa-V and Ps 1448a. . . 41

2.10 Deletion of genes from E. coli strains . . . . . . . . . . . . . . . . . . . . . 41

2.11 SDS-polyacrylamide gel electrophoresis . . . . . . . . . . . . . . . . . . . . . . . 42

2.11 .1 Molecular weight markers . . . . . . . . . . . . . . . . . 42

2.12 Artificially induced spontaneous streptomycin resistance mutation . . . . . . 42

2.13 Antimicrobial testing . . . . . . . . . . . . . . . . . . 43

2.13.1 Details of serial dilution plate set up, challenge and recording. . . . . 43

2.13 .2 Culture preparation . . . . . . . . . . . . . . . 44

2.13.3 Culture challenge and plate reading . . . . . . . . . . . . . . 45

2.14 Competitive growth fitness assay of antibiotic resistant strains . . . . . . . . . 45

2.14.1 Rich media based co-culture growth competition assay . . . . . . . . 45

2.14.2 Kiwifruit seedling based co-culture growth competition assay . . . . . 46 
3 Experimental Results - Antimicrobial Agents 48

3.1 Introduction . . . . . . . . . . . . . . . . . . . . . . . . 48

$3.2 \mathrm{IC}_{50}$ screening of antimicrobials $\ldots \ldots \ldots \ldots \ldots \ldots \ldots$

3.2 .1 Assay design . . . . . . . . . . . . . . . . . . . 49

3.2 .2 Assay preparation and optimisation . . . . . . . . . . . 50

3.2 .3 Candidate compounds . . . . . . . . . . . . . . . . . 51

3.2 .4 Results . . . . . . . . . . . . . . . . . 54

3.2 .5 Chloramphenicol and gentamicin activity . . . . . . . . . . 55

3.2 .6 In vitro activity of established field use antibiotics . . . . . . . . . . 56

3.3 A novel chemical inhibitor system of Psa-V . . . . . . . . . . . . . . 56

3.3 .1 Niclosamide . . . . . . . . . . . . . . . . . . . 56

3.3.2 Preliminary evaluation of nitroreductase activity in Psa-V . . . . . . 57

3.3.3 Efflux pumps and efflux pump inhibitors . . . . . . . . . . 58

3.3.4 Inhibition of Psa-V by niclosamide in combination with the efflux pump inhibitor $\mathrm{PA} \beta \mathrm{A} \ldots \ldots \ldots \ldots \ldots \ldots$

3.4 Development of antibiotic resistance in Psa-V . . . . . . . . . . . . . . 60

3.4.1 Antibiotic mechanisms and resistance . . . . . . . . . . . . . 60

3.4.2 Spontaneous streptomycin and kasugamycin resistance in Psa-V . . . 62

3.4 .3 Induced streptomycin resistance results . . . . . . . . . . . . . . . 62

3.4.4 A mutation in rpsL causes streptomycin resistance . . . . . . . . . . 63

3.5 Fitness burden of streptomycin resistance . . . . . . . . . . . . . . 65

3.5.1 In vitro competition assay design . . . . . . . . . . . . . . . . . . 66

3.5 .2 In vitro competition assay results . . . . . . . . . . . . . . . . 67

3.5.3 In planta . . . . . . . . . . . . . . . . . . . . . . 67

3.6 Discussion . . . . . . . . . . . . . . . . . . . . 70

4 Experimental Results - Psa-V Knockouts $\quad 73$

4.1 Introduction . . . . . . . . . . . . . . . . . . . . . 73

4.1.1 Biocontrol agent (BCA) modes of action . . . . . . . . . . . 73

4.2 Virulence mechanisms as targets for control . . . . . . . . . . . 78

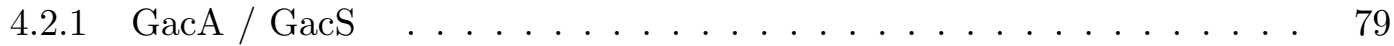

4.2 .2 Quorum sensing . . . . . . . . . . . . . . . . 79

4.2 .3 Type III secretion system (T3SS) . . . . . . . . . . . . . . . 80

4.2 .4 Psa-V effectors . . . . . . . . . . . . . . . . . . . . . 81

$4.2 .5 \quad$ NRPS gene cluster . . . . . . . . . . . . . . . . . 81

4.2 .6 Knock out target summary . . . . . . . . . . . . . . . 82

4.3 Experimental results . . . . . . . . . . . . . . . . . . . . . . 84

4.3.1 Gene truncation . . . . . . . . . . . . . . . . 84

$4.3 .2 \quad$ Absent overlap products . . . . . . . . . . . . . . . . 85

4.3.3 Vector selection . . . . . . . . . . . . . . . . . . 85

4.3.4 Transformation of Psa-V . . . . . . . . . . . . . . 86

4.3.5 Electroporation . . . . . . . . . . . . . . . . . 86

4.3 .6 Conjugation . . . . . . . . . . . . . . . . . . . 87 
4.4 Non-ribosomal peptide synthetase over expression in Psa-V . . . . . . . . . 89

4.5 Discussion . . . . . . . . . . . . . . . . . . . . . . . 90

5 Conclusions $\quad 92$

5.1 Research motivation . . . . . . . . . . . . . . . . . . . 92

5.2 Key findings . . . . . . . . . . . . . . . . . . . . . . . . 92

5.2 .1 Measuring anti-Psa-V activity . . . . . . . . . . . . . . 92

5.2 .2 Effective compounds . . . . . . . . . . . . . . . . . . 93

5.2.3 Potentiating the anti-Psa-V activity of niclosamide with an efflux pump inhibitor . . . . . . . . . . . . . . . . . . . 94

5.2 .4 Streptomycin resistance . . . . . . . . . . . . . . . . . . . . 94

5.2 .5 Psa-V knockout attempts . . . . . . . . . . . . . . . 95

5.3 Future directions . . . . . . . . . . . . . . . . . . . . . . . . 95

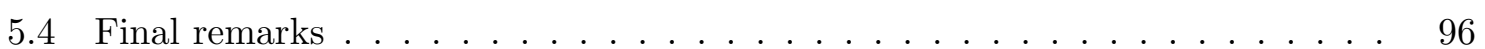




\section{List of Tables}

2.1 E. coli strains . . . . . . . . . . . . . . . . . . . . . 26

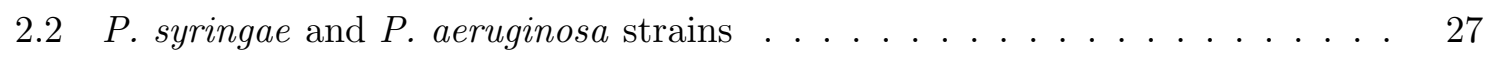

2.3 Plasmids used in this study . . . . . . . . . . . . . . . . . 27

2.4 Primers used in this study . . . . . . . . . . . . . . . . . . . . 29

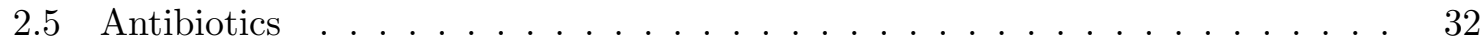

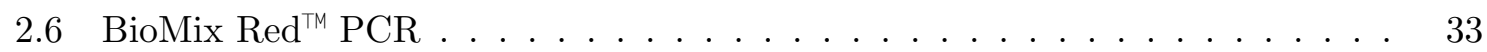

2.7 Standard BioMix Red ${ }^{\mathrm{TM}} \mathrm{PCR}$ thermocycler protocol $\ldots \ldots \ldots \ldots \ldots$

2.8 Tris Acetate-EDTA buffer . . . . . . . . . . . . . . . . . . . . . . 34

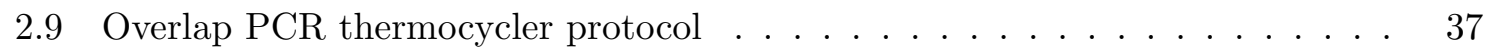

3.1 Candidate compounds showing efficacy in this study . . . . . . . . . 53

$3.2 \quad \mathrm{IC}_{50}$ results for tested compounds. . . . . . . . . . . . . . . . 55

4.1 Candidate genes as knockout targets . . . . . . . . . . . . . . . . 83

4.2 Successfully truncated genes . . . . . . . . . . . . . . . . 85

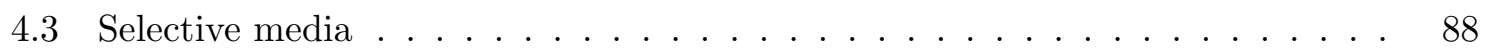




\section{List of Figures}

1.1 Copper spray on kiwifruit vines $\ldots \ldots \ldots \ldots \ldots$

1.2 Basal plant immunity . . . . . . . . . . . . . . . . . . 15

$1.3 \mathrm{H}_{2} \mathrm{O}_{2}$ kinetics in relation to plant immune response . . . . . . . . . . . . . . 15

1.4 Type III secretion needle complex _ . . . . . . . . . . . . . . . . 15

1.5 Susceptible host-pathogen interaction . . . . . . . . . . . . . 16

1.6 Resistant plant pathogen interaction . . . . . . . . . . . . . . 18

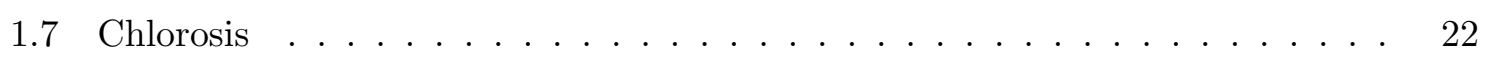

2.1 Splicing by overlap extension . . . . . . . . . . . . . . . . 36

2.2 Assay plate set up step $2 \ldots \ldots \ldots \ldots \ldots \ldots$

2.3 Assay plate set up step $3 \ldots \ldots \ldots \ldots \ldots$. . . . . . . . . . . . . . . . . . . . . . . . . . .

2.4 Test plate immediately prior to culture challenge. . . . . . . . . . . . . . . 44

3.1 Schematic of test plate set up . . . . . . . . . . . . . . . 50

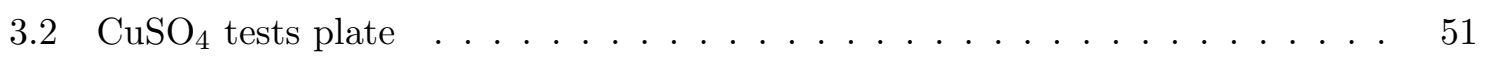

3.3 Oxytetracycline does response . . . . . . . . . . . . . . . . 54

3.4 Chlorine dioxide dose response . . . . . . . . . . . . . . . . . 54

3.5 Silver nitrate dose response . . . . . . . . . . . . . . . . . . 54

3.6 Niclosamide $\left(2^{\prime}, 5\right.$-dichloro-4'-nitrosalicylanilide $) \ldots \ldots \ldots \ldots$

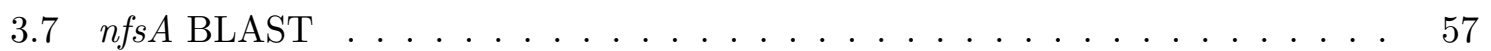

3.8 Nitrofurantoin growth inhibition . . . . . . . . . . . . . 58

3.9 Multidrug efflux pump nucleotide alignments . . . . . . . . . . . . . . 59

3.10 Niclosamide $\mathrm{PA} \beta \mathrm{N}$ heat map . . . . . . . . . . . . . . . . . . 59

3.11 Psa-V wildtype and P3-Strep ${ }^{\mathrm{R}} \mathrm{IC}_{50}$ shift $\ldots \ldots \ldots \ldots \ldots$. . . . . . . 63

3.12 rpsL alignment . . . . . . . . . . . . . . . . . . . . . . 64

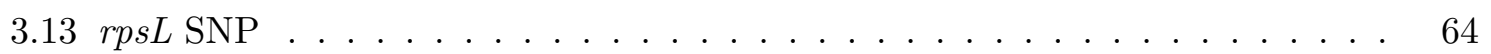

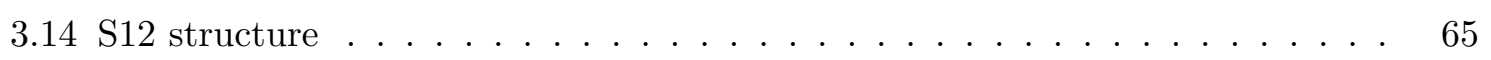

3.15 In vitro competition assay . . . . . . . . . . . . . . . . . . . . 67

3.16 In planta co-inoculation results . . . . . . . . . . . . . . . . . 69

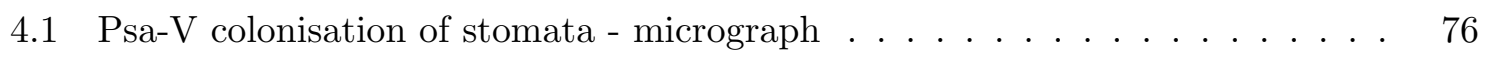

4.2 Overlap primer design . . . . . . . . . . . . . . . . . . 84

4.3 Overlap primer homology region . . . . . . . . . . . . . . . . 84

4.4 Overlap primer mismatches . . . . . . . . . . . . . . . . . 84

4.5 Knockout vector integration schematic . . . . . . . . . . . 86 


\title{
Chapter 1
}

\author{
Introduction
}

The overall focus of this study was to investigate control methods for use against a recently emerged bacterial pathogen of kiwifruit causing a global epidemic, Pseudomonas syringae pv. actinidiae(Psa). In this introduction, some of the shortcomings of the current methods used in attempts to control the pathogen are considered, then possible alternative chemical and biocontrol strategies are appraised. First however, to place the importance of this work in an appropriate context this introduction will begin with a review of the domestic and global kiwifruit industries, including an a reflection on the economic impact caused by the Psa epidemic. Throughout, the contributions from international research efforts on $P$. syringae genetics, which have guided much of this study, are also discussed.

\subsection{The host - Actinidia spp. - an industry, an identity, a monoculture.}

\subsubsection{Buds of industry: Cultivar development and global dispersal}

Kiwifruit (Actinidia spp.) have been an important economic crop in New Zealand for several decades with the main area of production centred in the Bay of Plenty (North Island). New Zealand's commercial kiwifruit production began here in 1937 when Mr Jim MacLoughlin planted a small block of Chinese gooseberries, the historical name for kiwifruit of this era. By 1940 he had converted all seven acres of his property to pursue their growing popularity. An escalating frequency of outstanding production obliged Mr MacLoughlin to facilitate development of the first export channels for Chinese gooseberries. By taking advantage of the regional port at Tauranga he increased the available market for himself and ultimately paved the way for the cultivation and commerce that remains in the area today [1].

Kiwifruit is a comparatively young crop, but the subsequent growth of the industry, here and abroad, has been impressive. Prior to the arrival of a virulent strain of bacterial canker to New Zealand in 2010, the export of around 100 million trays p.a., attracting earnings of ca. NZD\$1B each year, ranked kiwifruit as this country's second most valued horticultural industry, exceeded only by wine export earnings [2]. On a global scale, nearly 1.5 billion $\mathrm{kg}$ of kiwifruit were produced in 2011. New Zealand alone was responsible for more than one-third of this figure and kiwifruit production in New Zealand was only marginally rivalled in that year by Italy [3]. 
Just two sequentially selected cultivars underpinned the industry's rapid growth. Two prominent varieties, distinct from the genetically diverse vines found throughout China, were developed in New Zealand by directed breeding programs. The initial stimulation of the industry was came from demand for the improved and modernised green variety of the fruit that became known as the Hayward cultivar (full Latin binomial name: Actinidia deliciosa "Hayward") - named in recognition of Hayward Wright, a pioneering New Zealand horticulturist. The Hayward cultivar maintained a monopoly on cultivation until HortResearch introduced the new cultivar Hort16A (Actinidia chinensis "Hort16A"), a yellow-fleshed variety derived from further experimental breeding in 1992 with germ plasm from China.

Owing to its sweet flavour and smooth skin Hort16A has proven to be an even more valuable commodity than the green Hayward variety. Although other varieties have been and continue to be trialled, these New Zealand developed cultivars provided the primary basis for the international kiwifruit industries in Italy, China, Chile, France, Greece and Japan, and are the reason for New Zealand becoming synonymous with kiwifruit $[4,5]$. Data from the UN Food and Agriculture Organisation (FAO) gives a global kiwifruit production of $1,489,900$ tonnes in 2011 . $29 \%$ of this is produced in Italy, $28 \%$ in New Zealand and $16 \%$ in Chile. The FAO data does not include values for production in China, which by other reports is the second largest producer of kiwifruit [6]. Because of its superior flavour and long storage life, Hayward has been the cultivar of choice throughout the world (97.5\% of kiwifruit plantings outside of China were at one stage Hayward and it is still the most popular green variety grown today) [7].

\subsubsection{The genetic bottleneck}

Because commercial kiwifruit supplies have relied so heavily on the two varieties mentioned above, very little genetic diversity is exhibited within the cultivated population of these species. Thus, each individual vine is equally vulnerable at the genetic level to the severe impact from an incursion of a single successful phytopathogen [8], as seen with the re-emerging highly virulent strain of $P$. syringae.

\subsection{The pathogen - Pseudomonas syringae pv. actinidiae: the causative agent of bacterial canker of kiwifruit}

\subsubsection{P. syringae - classification and description}

The introduction to the pathovar that is the the primary focus of this thesis, will begin one level above its position in the hierarchical classification system used to resolve its relationship to the species $P$. syringae as a whole. P. syringae is a species that has been historically damaging to horticultural practices and is an archetypal plant pathogen $[9,10]$.

Members of the $P$. syringae species are Gram-negative phytopathogenic bacteria which are known for their diverse range of specific host interactions [11]. Some defining characteristics that are useful in identifying the species include an obligate aerobic metabolism and a motile rod-shaped cell with multiple polar flagella (singular flagellum). 
Because of its widespread prevalence as a horticultural pest that causes a range of disease symptoms affecting an array of cultivated crops $P$. syringae was once recognised as more than 40 separate species. These have now been collated into a single species complex which is divided into 57 known pathovars (pv. - a pathovar is an infrasubspecific epithet used to distinguish between bacterial strains within a species, usually based on the exhibited host-specificity across diverse plant hosts) $[12,13]$. For P. syringae, the naming of pathovars is generally derived from the primary host in which they cause disease. For example P. syringae pv. phaseolicola causes the halo blight disease of the common bean. The bean host is denoted by the botanical title Phaseolus vulgaris, hence the strain's pathovar is phaseolicola [14].

Individual pathovars will generally cause disease in only one, or sometimes a few, specific hosts. Confounding this naming convention, some P. syringae pathovars as part of their normal lifecycle are also found to exist commensally on host species in which they do not cause disease symptoms, acting as a reservoir for future infection to liable hosts. For example, the type strain P. syringae pv. syringae has 80 listed hosts, giving it the largest known host range, yet disease symptoms only manifest on syringa, prunus and phaseolus host species [9]. The 57 pathovars of P. syringae described to date have been divided into nine discrete genomospecies by Gardan, Shafif \& Grimont (1997) [15] using DNA-DNA hybridisation and ribotyping. This high-fidelity molecular based classification was a significant step in understanding the pathogenicity and evolution of the P. syringae species complex, as related pathovars frequently share conserved virulence factors.

\subsubsection{P. syringae: lifecycle.}

Most $P$. syringae invade and damage plant cells, but importantly, as part of their natural lifecycle they also have the ability to epiphytically colonise the surface of host plant leaves in a metabolically restricted, non-virulent state. Depending on the host, the environmental conditions and the bacterial load, metabolic changes regulated in part by quorum sensing can rapidly switch the bacterial colony from a non-virulent state into a virulent one, in which they will invade the host and cause disease symptoms $[16,17]$. In the virulent state $P$. syringae pathovars are among the most destructive bacterial phytopathogens known, therefore developing strategies to control epidemic strains of $P$. syringae is a major agricultural concern.

\subsubsection{Pv. actinidiae}

One particularly damaging pathovar, not included in the original categorisation by Gardan et al. (1997) [15], but subsequently placed into genomospecies 8 together with Pseudomonas avellanae (previously P. syringae pv. avellanae) and P. syringae pv. theae is $P$. syringae pv. actinidiae (Psa) [18]. This pathovar specifically infects kiwifruit vines, and can do so effectively that it has potential to cause major economic damage to New Zealand's kiwifruit industry.

On the basis of LOPAT tests Psa is oxidase negative due to the lack of aa3-type cytochrome c oxidase [19] and arginine dihydrolase negative because of the lack of an 
arginine dihydrolase system [20]. LOPAT tests are used to identify different pathovars of $P$. syringae by metabolic profiling, the series of determinative tests used in LOPAT identification are: L, levan production; O, oxidase production; P, pectinolytic activity; A, arginine dihydrolase production; and $\mathrm{T}$, tobacco hypersensitivity [21].

\subsubsection{First isolation}

Psa was first described as causing a bacterial canker disease of green kiwifruit in Japan in 1989 [22]. Subsequently in 1994, reports were made of bacteria matching the LOPAT profile of Psa being isolated from Hayward kiwi fruit vines in Italy. These isolates were further characterised as Psa by multi locus sequence typing (MLST) and BOX-PCR. This strain only caused limited economic losses in Italian kiwifruit orchards so these incidents did not cause widespread concern at the time, a genetically similar strain did cause heaver loses in Korea and Japan with the difference in impact accredited to different environmental conditions. However, from 2007-2008 a serious epidemic outbreak of bacterial canker on kiwifruit vines in central Italy was found to be caused by a new highly virulent strain of $P$. syringae pv. actinidiae [23]. The most conspicuous symptoms of the infection were swollen regions on stems, known as cankers, which emitted a red exudate due to the heavy bacterial load within the vascular system of the vines.

Central Italy is a globally significant area of kiwifruit production and this outbreak had severe effects on production within the first season. Almost all of central Italy's 800 hectares of A. chinensis (gold kiwifruit) orchards have now been destroyed, placing the sustainability of the Italian industry in jeopardy. Neither intensive copper use nor "slash and burn" tactics were able to contain the outbreak, and no alternative control methods have been found. As will be discussed further below, this epidemic strain was found to be genetically distinct from the original isolate of Psa recovered from the same area in 1994 $[24,25]$. Although the latest epidemic strain has been found to cause disease in all commercial strains of kiwifruit, the variety with the highest economic importance, cultivar Hort16A, still appears the most susceptible [20].

The following quote comes from an article authored by Everett, KR et al., (2011) [26]: "It is estimated that economic losses of $€ 2 \mathrm{~m}$ will result from the epidemic in Italy. If the strain in New Zealand is confirmed to be the same as that causing this recent outbreak in Italy, then, depending on climatic differences, economic losses can also be expected".

\subsubsection{Psa in New Zealand}

As New Zealand also maintains an economically significant kiwifruit industry, reports of the severity of the outbreak in Italy alarmed kiwifruit industry stakeholders in this country. Scientists at Plant and Food Research New Zealand proactively developed a polymerase chain reaction (PCR) based test, designed to be as specific as possible in detecting Psa, to allow early detection should the pathogen cross bio-security borders into New Zealand. The specificity of this test was based on the 16S23S rDNA intertranscribed spacer sequence of Psa [27]. In November 2010, symptoms of necrotic lesions on leaves, wilting and shoot die-back, typical of the early stages of bacterial canker in Actinidia spp., were observed for 
the first time in New Zealand on Hort16A plants growing in Te Puke. Isolates from infected leaves were identified as Psa positive using the PCR test [27]. Further field testing and analysis showed this primer set was not as specific as had been hoped and could gave false positives results, i.e. not only returning positive results for the highly virulent Psa, but also a much less virulent strain that already existed in New Zealand. These strains were labeled at the time as Psa-V to denote the high virulent strain and Psa-LV to denote the low virulent strain. The assay also returned positive results from two other $P$. syringae pathovars of the same genomospecies, although these do not cause any symptoms on kiwifruit vines. Consequently, the industry had to employ real - time PCR melting point analyses as a second test to delineate between the two symptomatic strains (Psa-V or LV) whenever a positive isolate was found. Ultimately, $99 \%$ of the isolates tested from the main areas of infection were found to be Psa-V (John Mackay, Technical Director, dnature molecular diagnostics, personal communications). During the timeframe of this study full genome analysis data from both Psa-V and Psa-LV became available, which allowed more effective Psa-V specific primers to be designed. Psa-V is now used as a general term to refer to any of the highly virulent emergent strains from different geographic location related to the current epidemic.

Psa-V isolated from New Zealand displayed identical molecular features in (MLST) analysis and linkage disequilibrium tests to the population of the highly virulent pathogen found in Italy $[28,29]$. Based on this information, the populations were at first assumed to be clonal between the two regions. However, recent sequence analysis comparing three high virulence Psa-V isolates; Psa-NZ a New Zealand isolate, Psa-IT2 a post-2008 isolate from Italy and Psa-CN1 a Chinese isolate - shows that Psa-NZ is essentially clonal to Psa-CN1 whereas Psa-IT2 differs by 10000 to 20000 nucleotide positions. The variation between Psa-NZ/CN1 and Psa-IT2 occurs primarily within a region of sequence that is rich in various pathogenicity genes and is bordered by the tRNA signatures of mobile elements [30]. This region resembles a pathogenicity island from P. syringae pv. phaseolicola. All three isolates mentioned containing there affiliated ICE'S are of comparable virulence and are all referred to as $\mathrm{Psa}-\mathrm{V}$, although it is possible that they may prove to respond differently to control measures (Assoc. Prof. Russell Poulter, personal communication). A more in-depth discussion on Psa-V phylogeny, looking at the similarities and differences between the geographic isolates, is covered in section (1.6).

As of 9 January the 2014, 78\% of New Zealand's total kiwifruit orchards have become infected with $\mathrm{Psa}-\mathrm{V}$ and new infections are still being detected on a weekly basis. This has increased from $\sim 50 \%$ in September 2012. As a measure to control the future spread of Psa-V in New Zealand the Ministry of Primary Industries (MPI) has approved, under the Biosecurity Act of 1993, strict zoning regulations on kiwifruit orchards, depending on the orchard's regional location and the Psa-V status in that area. Three zones are identified under this scheme known as the National Psa-V Pest Management Plan (NPMP). All kiwifruit orchards nationwide are categorised into either one of the following zones: 
Exclusion region; Psa-V not present in this area.

Containment region; Psa-V detected at low levels in this area.

Recovery region; Psa-V detected at high levels in this area.

Strict regulations on orchard practices and movement of plant material are enforced in these zones.

At the time of writing Recovery regions are in place for:

$\begin{array}{lll}\text { Franklin } & \text { Katikati } & \text { Opotiki } \\ \text { Tauranga East } & \text { Tauranga West } & \text { Te Puke } \\ \text { Waihi } & \text { Waikato Whakatane } & \end{array}$

At the time of writing Containment regions are in place for:

Coromandel

Hawkes Bay

Poverty Bay - Gisborne area Kerikeri

The remainder of the country's kiwifruit growing areas are considered Exclusion regions.

New Zealand regulatory authorities (Agricultural compounds \& Veterinary Medicines, a division of MPI) have for the first time given approval for limited application of KeyStrepto $^{\text {TM }}$ (the aminoglycoside antibiotic drug streptomycin), and subsequently a second antibiotic with the tradename Kasumin ${ }^{\text {TM }}$ (kasugamycin) on kiwifruit orchards within restricted zones. Approval must be applied for and granted before either antibiotic can be used on orchards. Only post-flowering non-producing blocks can be approved for antibiotic spray programs to eliminate antibiotic residue in the fruit and reduce any contamination of honey.

Another aspect of control measures being used is the introduction of the cultivar "Gold3", a new variety of gold kiwifruit developed recently in a collaborative breeding program between ZESPRI and Plant and Food Research Ltd. This variety is purported to have some intrinsic resistance to Psa-V, although laboratory tests have publicly confirmed Psa-V positive test results from "Gold3" vines in a kiwifruit orchard in Whenuakite in the Coromandel [5]. Moreover, microscopic analysis indicates no difference in the progression of infection between "Gold3" and "Hort16A" [31]. The cost of orchard conversion to the new cultivar is substantial. Per hectare, replacing "Hort16A" with "Gold3" is estimated by MPI to total $\$ 33,100$ including the licence fees to grow this cultivar. So far 2000 hectares of kiwifruit orchard has been converted at a cost of more than $\$ 65 \mathrm{M}$.

Altogether the economic impact of Psa-V in New Zealand has already been severe. Although it is difficult to decide how to calculate an actual figure of the costs caused by the infection thus far, the SunLive newspaper reported that $\$ 450,000$ per canopy hectare [for "Hort16A" orchards] last season is now worth about $\$ 60,000$ to 80,000 per canopy hectare, virtually a bare land price [32]. On top of these direct losses by the orchardist are the wider costs of industry and government budgets for control programs and research. A report of the overall economic impact of the outbreak in New Zealand has been prepared by a Lincoln University consultant. The main findings of the report state that Psa-V is expected 
to cost the kiwifruit industry between 310 and 410 million dollars in net present value terms over the next five years. Over a ten year time horizon the estimated costs will increase to approximately 500-600 million dollars and over a 15-year period to between 740-885 million dollars [33]. Thus, there is a strong financial incentive to investigate new and effective methods to control the spread and infectivity of Psa-V. In addition to the work described in this thesis, funding from the Ministry of Research, Science and Innovation (now Ministry of Business, Innovation and Employment) in partnership with Seeka Kiwifruit Ltd has supported the ongoing efforts to sequence the genomes of several New Zealand Psa-V isolates. Draft sequence data from the earlier efforts became increasingly available during the course of this study, subsequently a complete Psa-V genome with extensive annotation was kindly supplied by Dr. Matt Templeton of Plant and Food Research for use in this study and at the time of writing a complete circularised genome had been published and deposited online [20].

\subsubsection{Effects of Psa-V infection}

At the apogee of infection Psa-V rapidly invades the primary vascular system of kiwifruit vines while metabolising the simple sugars transported through vessels. Bacterial growth at this stage is to such an extent that vessels become blocked, new shoots die-back and the plant collapses. When stems are cut in the later stages of infection a thick bacterial ooze exudes from the larger vessels. The bacteria gain entry to the plant via the small openings of the lenticels and stomata, then move through the cortex and into the phloem region by breaking down cell walls. To enable this Psa-V contains genes that are often associated with other woody-host pathogens. These genes seem to allow Psa-V to utilise unsubstituted lignin-related compounds and other plant derived phenolic compounds, such as mandalate and phenol [34], which leads to the complete breakdown of woody structures and produces weepy bludging cankers on the stems.

Canker formation with exudate indicates that the intercellular spaces are also colonised by the bacteria. This is backed up with micrographs showing Psa-V in the inter-cellular spaces of the outer-cortex parenchyma and Psa-V breaking through cortical fibers into the inner-cortex.

The exudate from cankers contains saturating levels of bacteria and promotes the spread of infection by rapid dissemination of viable bacterial cells. [9, 35]. Rain and wind, animals, insects and standard orchard practices such as pruning may all be contributing vectors.

Together the dense colonisation of vessels and the degradation of lignified cells lead to plant death within one growing season; and the rapid spread of Psa-V can mean entire orchards are lost. This has been the case wherever Psa-V has been found, as no effective control measures are yet available. 


\subsection{Control of Psa}

\subsubsection{Previously trialled and current control methods}

Although no substantial Psa-V control measures are yet available to kiwifruit growers, several options have been trialled including orchard practices that may allow for a sustainable kiwifruit industry in co-existence with the pathogen. The methods most widely implemented have involved spraying antimicrobial compounds onto the vines and leaves of the plants as a prophylactic measure. The Kiwifruit Vine Health (KVH) recommended spray programme specifies spraying copper based compounds throughout the season as the best practice of Psa-V control [36]. However, this recommended use has not worked to reduce the damage caused by $\mathrm{Psa}-\mathrm{V}$.

Control regimes employed in Japanese and Italian orchards prior to the $2008 \mathrm{Psa}-\mathrm{V}$ outbreak relied almost entirely on spraying vines with copper compounds. Initially in Japan, where Psa was first described as a pathogen, all strains were found to be sensitive to low levels of copper. However, several years of routine copper applications promoted the emergence of a resistant strain in Japan. Copper resistance in this case was carried on a plasmid containing six open reading frames (ORFs) that have homology to the genes $\operatorname{cop} A$, $\operatorname{cop} B, \operatorname{cop} C, \operatorname{cop} D, \operatorname{cop} R$ and $\operatorname{cop} S$. These six genes make up the resistance-operon present in copper resistant strains of $P$. syringae pv. tomato [37, 38].

Psa-V also contains chromosomal genes encoding for copper transport and detoxification proteins. Unsurprisingly, the use of copper compounds had little effect as a first line of defence to control the Psa-V outbreak in Italy. Indeed, in many cases copper use there proved worse than futile, with little alleviation of infection and often severe damage resulting from the phytotoxic levels of copper applied to some orchards. In New Zealand field trials, conducted by KVH, some level of phytotoxicity was observed with all copper treatments tested (Fig.1.1).

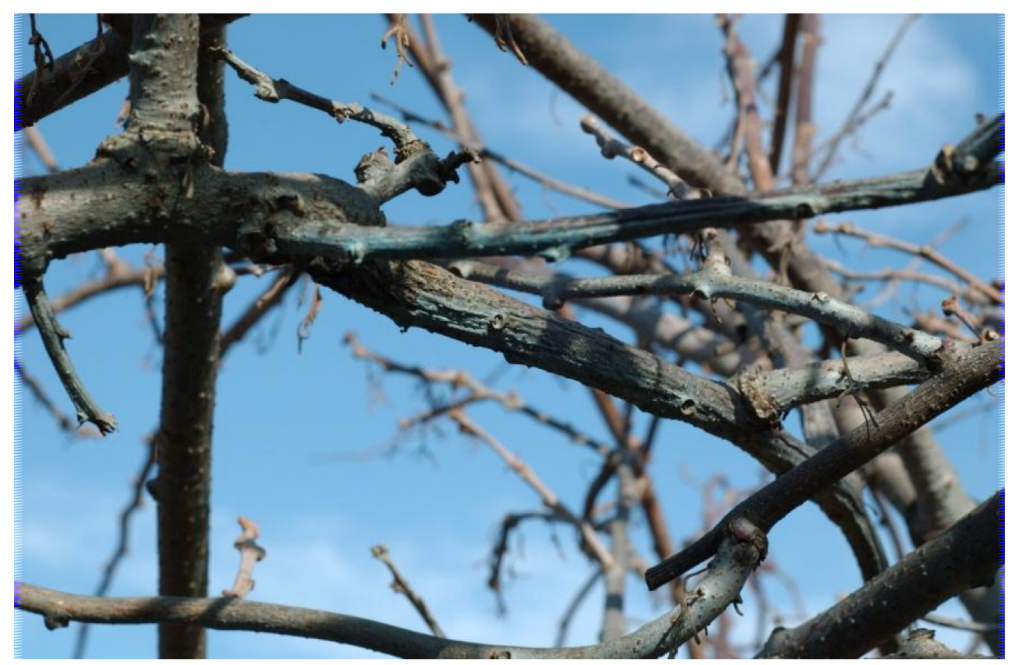

Figure 1.1: Kiwifruit vines left blue from heavy copper spraying in Psa-V infected orchard. KVH online publication Psa and Italian Kiwifruit Orchards, Kay (2011) [39] 
All current management techniques for bacterial agricultural pathogens in New Zealand, which consist mainly of applying a variety of copper compounds or other heavy metals, have been ineffective in the field against the Psa-V epidemic. Unfortunately, the options for controlling $P$. syringae during epidemic outbreaks are of limited number and effectiveness, and have always been a major unresolved agricultural issue [40]. Spray regimes using antibiotics have been proposed as a last line of defence in this country. Limited use of streptomycin and subsequently kasugamycin has been approved by the environmental protection authority (EPA). Antibiotic use on kiwifruit vines may lend some level of prophylactic control if used effectively before a Psa-V infection occurs, but this would require a heavy spray regime that effected a continuous repression of $\mathrm{Psa}-\mathrm{V}$ populations in the orchard. This is because the spraying of bactericides can only control a Psa-V infection by dampening or eliminating epiphytic populations before they reach high epiphytic density and become virulent. Once the virulent pathogen has penetrated into the leaves or stems of the plant, whether or not any symptoms are expressed, it is impossible for either coppers or antibiotic sprays to reach it $[40,41]$. Of concern, resistance to streptomycin has arisen when the antibiotic has been used in the past to defend against various phytopathogens including Psa [42]. Due to this resistance problem the anticipated longevity of streptomycin as a useful bactericide is low. To reduce the risk of resistance a second antibiotic, kasugamycin, was registered to give another option for Psa-V control. The probability of bacteria simultaneously developing resistance to multiple antibiotics when applied in concert is exponentially lower than that of resistance to an individual antibiotic arising. However, there is concern that Psa-V in New Zealand may develop resistance to each antibiotic in turn, as the guidelines issued to orchardists approved to use antibiotics do not recommend simultaneous application of each antibiotic. Rather, they state that only two consecutive applications of a single antibiotic may be used, then an alternative, either a different antibiotics or another bactericide, must be applied if the grower needs to continue spraying. There are no guidelines requiring the application of both antibiotics simultaneously and growers are limited in the number of times these antibiotics can be used.

Not only is there the high likelihood of bacterial resistance developing, but there is also the risk of antibiotic residues entering the food chain with possible impacts on human health, as these are drugs usually reserved for human health therapy and pathogen resistance from the environmental to these drug may occur also the antibiotics may have toxicity issues for humans consuming them at high levels in food. As such, the current prophylactic use of antibiotics is generally viewed as a short-term scenario at best. There are also economic concerns for growers using antibiotics, for example, the European Union restricts the import of fruit containing any antibiotic residues [43]. Finally, bee keepers in kiwifruit growing areas have concerns that streptomycin or kasugamycin may be transferred to honey products. For all of these reasons, registration of antibiotic use in kiwifruit orchards has met some resistance, with many stakeholders raising concerns over their use.

In order to mitigate the build-up of antibiotic residue in the food chain and to conserve the export markets of kiwifruit and honey, MPI has imposed restrictions on streptomycin and kasugamycin application. The Ministry's regulations dictate (at the time of writing) that spraying must be confined to limited concentrations on non-producing vines, and have 
banned injecting vines in an attempt to reduce antibiotic residues in fruit. Desperate growers ignoring these restrictions have focused media attention on negative scenarios associated with antibiotic use [44].

\subsection{Proposed alternative control measures}

New control measures to expand the arsenal against Psa-V are desperately sought by the industry. Two possible alternative strategies to combat Psa-V are of particular relevance to this study.

\subsubsection{New bactericidal compounds effective against Psa-V}

The kiwifruit industry has fast-tracked the testing of products in the search for a compound or formula that may be useful in controlling Psa-V in the field. In vitro testing has highlighted some products that have strong antimicrobial effects on Psa-V [45]. However, in the field these products are still limited by reliability, the likelihood of acquired resistance, phytotoxicity and, in almost all cases, a lack of systemic effects. Among the compounds tested were the classic antibiotics, several commercially available copper compounds, natural products, other antimicrobial compounds and a family of plant protective elicitors. Some biocontrol agents were also tested. The 2013-2014 crop protection programme issued by Zespri lists tested products now approved for Psa-V control. Including the streptomycin and kasugamycin products and three copper formulations, in total only eight options are listed for use on gold kiwifruit and one extra for use on the green varieties. The other non-antibiotic/copper products listed are: a plant growth regulator - forchlorfenuron; an elicitor - acibenzolar-S-methyl; and a biocontrol agent Ulocladium spp. All of these options only carry an agricultural compounds \& veterinary medicines limited claim as they have not been yet been fully proven.

\subsubsection{Biological control by antagonistic micro-organisms}

Plant disease control using non-pathogenic microorganisms to antagonise infectious strains is a well-developed concept [46]. The strains used for this purpose are called biological control agents (BCA). There are several examples of proven BCA systems that are in use with crops on a commercial scale. A few examples are listed below to validate this concept.

\section{Crown gall control}

The non-pathogenic strain Agrobacterium radiobacter K1026 is used to control crown gall, a bacterial disease of commercially important plants in the Rosaceae family (this includes stone fruit, apples and ornamental roses) which is caused by pathogenic strains of Agrobacterium tumefaciens. The K1026 strain is licensed as the pesticide NOGALL ${ }^{\mathrm{TM}}$ and its registration was the first case of an intact genetically engineered organism released for commercial use [47]. 


\section{Fire blight control}

Controlling fire blight disease of pear, caused by the bacteria Erwinia amylovora, can be achieved by several possible antagonist strains. Bacillus subtilis QRD-137, P. fluorescens A506, and Pantoea agglomerans C- 91 have all shown commercial application to control the disease caused by Erwinia amylovora by inhibiting the population expansion of the pathogen on flower buds $[48,49]$.

\section{Fungal disease control by Pseudomonas}

Non-pathogenic strains of fluorescent $P$. syringae have been used with success against fungal diseases, in certain cases resulting in up to $100 \%$ disease control $[50,51]$. Likewise, the plant-growth-promoting rhizobacterium (PGPR) P. aeruginosa 7NSK2 improves the growth of several crops when present in the soil by protecting the plants against fungal root diseases [52], for example, it is an efficient antagonist of Pythium-induced damping-off, a fungal disease of tomato seedlings [53].

\section{P. syringae pathovars}

The antagonism of $P$. syringae pathovars of the phylloplane (leaf surface habitat) themselves by other BCAs is not as well refined as in previous examples and coverage in the literature is limited. This may simply be due to the $P$. syringae pathovars being such well adapted pathogens $[54,55]$. Several BCAs have been included in field trials on kiwifruit, however no conclusive results or lasting protection have been seen. Research from field trials in Italy showed a general low efficacy for commercially available BCAs to control Psa-V but did report the possible application of strains isolated from kiwifruit flowers to protect the vines during blooming.

\subsubsection{Possible biocontrol of Psa-V}

To control Psa-V in kiwifruit this antagonising effect would ideally happen on the surface of the leaves since the incidence of diseases caused by $P$. syringae can be correlated with the density of the epiphytic population. Two approaches could be taken to isolate an antagonising strains to be used as a BCA: (i) in vitro screening followed by in vivo trials of naturally occurring micro-organisms that colonise the same environment for antagonistic properties; or (ii) development of a closely related strain of the pathogen, through genetic manipulation or laboratory evolution, that does not cause disease but is able to compete and occupy the same niche and perhaps incite the defensive state in the plant. It is this latter approach that was a driving force behind much of the research conducted in this thesis. However, to appreciate possible means by which a non-pathogenic isogenic derivative of $\mathrm{Psa}-\mathrm{V}$ might be generated by genetic manipulation, it is first necessary to gain insight into the genetic basis by which Psa-V is able to cause disease in kiwifruit plants. 


\subsection{Plant immunity and pathogen-host interactions}

In recent years it has become apparent that the interplay between plant host and phytopathogenic bacteria is very complex and the details of the actions of many of the components are still being elucidated. The current paradigm holds that there are two distinct phases of plant immunity -one phase that is a broad-spectrum response to microbe exposure in general and another acute response that is host-pathogen-specific. However, it has been argued that the triggers and appropriate responses of each stage exist on a continuum with overlapping outcomes so this distinction cannot be strictly maintained [56]. For the sake of clarity, this overview will examine the two phases separately, as the distinction between the phases is always example-specific.

\subsubsection{Basal plant immunity}

Plants can mount a defensive immune response to the micro-organisms that they are exposed to in the environment. The initial step is plant recognition of a micro-organism's presence. To be pathogenic, most bacteria must invade the interior of the plant and in doing so will contact plant cellular membranes. When this occurs, plant cell membranes become exposed to the pathogen-associated molecular patterns (PAMPs) of the invading cell. PAMP is the categorisation given to a diverse range of microbially derived molecules, or parts thereof, that can be recognised by the host plant [57]. PAMPs are usually widely conserved molecules found in nearly all bacteria as they make up essential cellular components and confer strong survival and reproductive advantages. They are also synonymously termed microbe-associated molecular patterns in recent literature as they are not pathogen-specific but are in fact intrinsic to entire classes of microbes [58, 59]. The naturally selection of successful microbial immunity has resulted in plants recognising evolutionarily important microbial molecules as PAMPs because pathogenic microbes have been unable to alter these molecules to avoid detection. The pathogens must maintain these indispensable molecules even though they will exert a negative selection pressure on the pathogen when they alert the plants immune system to its invasion. In bacteria, vital molecules such as bacterial lipopolysaccharides, flagellin, elongation factor Tu (EF- Tu), cold-shock protein and peptidoglycans are all recognised by the plant as PAMPs [60, 61]. Although many PAMPs exist, the most commonly cited and well characterised example is flagellin, the conserved subunit of the bacterial flagellum [62].

The plants receptors that recognise PAMPs are called pattern recognition receptors (PRRs) and these transmembrane protein receptors are exquisitely sensitive to the chemical signals that trigger them [57]. The plant receptor for flagellin, the archetype PAMP characterised in the model organism Arabidopsis thaliana, is Flagellin Sensitive-2 (FLS2) a leucine-rich repeat transmembrane receptor kinase [63]. Homologs of FLS2 have been found in all higher plants for which genomic information is available suggesting plants have a long history of recognising flagellin as a PAMP [59].

Recognition of a pathogen-presented PAMP by the PRR of the host cells results in PAMP-triggered immunity (PTI), which is the cumulative outcome of the first stage in a plant immunity response. The PTI response is a broad spectrum basal microbial resistance 
resulting from the up-regulation of about 1,100 genes. Transcriptome studies in A. thaliana indicate that the recognition of many different PAMPs by the plant can result in a sudden and equivalent regulatory effect on a very similar subsets of the plant immunity and microbial defence genes, i.e. the plant induces approximately the same PTI response to different pathogens $[64,65]$. This change in gene regulation caused by the PTI response first creates a transient antimicrobial environment within and around the infected cell to halt further colonisation [66]. Following a successful pathogen recognition, the antimicrobial environment is established by a suite of cellular defence responses, the most immediate response being the rapid production of reactive oxygen species (ROS) at the site of infection, referred to as the oxidative burst [67]. Further investigation of the PTI has shown that ROS, which include super oxide $\left(\mathrm{O}_{2}^{-}\right)$and its dismutation product hydrogen peroxide $\left(\mathrm{H}_{2} \mathrm{O}_{2}\right)$, are produced apoplastically via the consumption of oxygen by enzymes, including plasma membrane NADPH-oxidases and cell wall peroxidases [68]. These ROS are antimicrobial in their own right, but the evidence also points to ROS playing a signaling role in the overall cellular immune response.

\section{Cell wall thickening}

Another plant defence mechanism that is mediated by PTI, usually hours to days later than the oxidative burst, is the thickening of the plant cell wall which is thought to create a physical barrier to invading pathogens. ROS are able to cause cell wall thickening directly by inducing membrane damage [69] or by the cross-linking of cell wall glycoproteins [70]. Depositions of callose, a major component of plant cell walls [71], can be visualised in $A$. thaliana after treatment with a synthetic PAMP called flg22. Flg22 is a synthetic peptide that imitates a conserved domain of the bacterial flagellin protein and it is used extensively in characterising PAMP recognition and the PTI system [72].

\section{Gene regulation and signaling}

Abundant attention to the up-regulation or down-regulation of gene expression and the detailed molecular processes of intercellular signaling during a PTI event has progressed as the needed tools have become accessible [73-75]. From these investigations there has been the realisation that within a certain plant the gene regulation and signalling pathways governed by the PTI response are noticeably the same irrespective of the vast possibilities of PAMP variations triggering them, even though each different PAMP will be detected by a unique receptor. Given that a convergent signaling network would be necessary to relay the activation signal from the many possible PAMP / PRR pairs on the plant cell membrane through the cytoplasm into the nucleus and then regulate a common schedule of defence genes [59], the mitogen-activated protein kinases (MAPKs) constitute prominent pathway candidates as they can facilitate this signalling pattern. Identifying a complete plant MAPK pathway, Asai et al., (2002) [76] determined that WRKY29, FRK1 and GST1 mRNA levels were increased via this pathway in A. thaliana protoplasts within $30 \mathrm{~min}$ of flg22 treatment. The action of these regulatory factors may be responsible for the further changes in gene expression that is required for a defensive response. The known plant 
defence proteins PAL1, GST1, PR1 and PR5 were also expressed after flg22 treatment. In another study, the WRKY transcription factors were activated by flg22 and they had positive feedback on their own transcription. WRKY transcription factors bind to W-box sequences in plant gene promoters increasing or decreasing gene transcription levels [77], W-boxes are common in the promoters of plant defence genes [78].

\section{Systemic acquired resistance}

In addition to the sudden local antimicrobial environment elicited by cellular PAMP recognition, the PTI response can also include a systemic component, which elicits a defensive state throughout the host plant (figure 1.2). This factor may be one important mechanism for the effective action of BCAs and is the basis of the protection provided by the plant elicitor family of pesticides. The integrated action is mediated in part by phytohormones such as salicylic acid (SA), gaseous ethylene and jasmonic acid. The release and build-up of these hormones during the PTI response drives a signal to distal regions of the plant to express pathogen related proteins (e.g. the proteins PR1 and PR2) that have a role in microbial defence. The expression of these proteins throughout the plant results in plant-wide-systemic acquired resistance (SAR) [79]. Upon pathogen attack the abundance, composition and schedule of the phytohormonal concoction created by the plant can differ between plant species, although the accumulation of SA is purportedly the most recognised link between PAMP recognition and SAR. Some pathogens may interfere with aspects of the hormonal response to alter it in favour of pathogenicity [80].

Again in A. thaliana, treatment with flg22 or a mutant strain of $P$ syringae pv. tomato DC3000 hrc C devoid of a type III secretion system (T3SS), and therefore unrecognisable to the plant's second-line defence processes, was found to drive an increase of SA. This highlights the interplay between PAMP recognition and SAR [81]. The observation that treatment with either flg22 or SA induced or suppressed a common set of genes led to the hypothesis that an interaction(s) between PAMP and SA signalling exists. Subsequent testing by the same group demonstrated that SA accumulates after flg22 treatment in an SID2-dependant manner (this plant enzyme is responsible for SA production), that the elevated level of SA affects transcriptional regulation of certain genes, and that SA is important for PAMP-triggered resistance [81]. This is because flg22 treatment failed to induce the expected wild-type resistance against $\mathrm{P}$ syringae pv. tomato DC300 hrcC in non-SA-producing $\triangle S I D 2$ mutant plants. The analysis of leaves by gas chromatography-mass spectrometry from treated plants show SA accumulation locally at the site of infection and systemically in disant tissues [80]. Production of SA was found to be an absolutely necessary intermediate to induce SAR in experiments performed by Durrant and Dong., (2004) [82]. SA plays an integral part of SAR induction but the actual mobile systemic signal to distal region of the plant under attack is yet to be definitely identified [83]. The preceding and final discussion point on PTI will be important to contemplate when considering possible mechanisms by which a BCA for Psa-V may work, and it therefore may advise aspects of BCA design. 


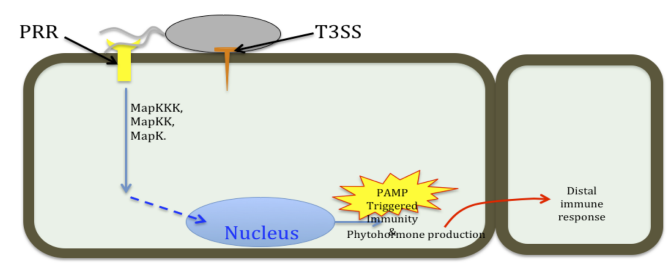

Figure 1.2: Basal plant immunity: In this schematic the pattern recognition receptor (PRR) recognises the flagellin of an invading pathogen and the PAMP-triggered-immune (PTI) response is activated. MapKKK -mitogen activated protein (Map) kinase kinase kinase, MapKK - Map kinase kinase, MapK - Map kinase. Collectively, this pathway represents a signalling cascade that can transduce external signals to the nucleus leading to a wide range of cellular responses via transcriptional regulation.

\subsubsection{Basal plant immune response suppression by pathogen secreted effectors}

The next phase of defence does not differ much in the environmental conditions created by the infected cell but is more sustained and robust (Figure 1.3), and triggered only by attacking pathogens that express certain virulence factors known as effectors [70]. Pathogens that can survive the defensive mechanisms induced by PTI typically have a repertoire of effector proteins that are injected in an unfolded state by a molecular needle complex (Figure 1.4), known as the Type III secretion system, directly into the plant cell cytoplasm where they become active in immune suppression [84].

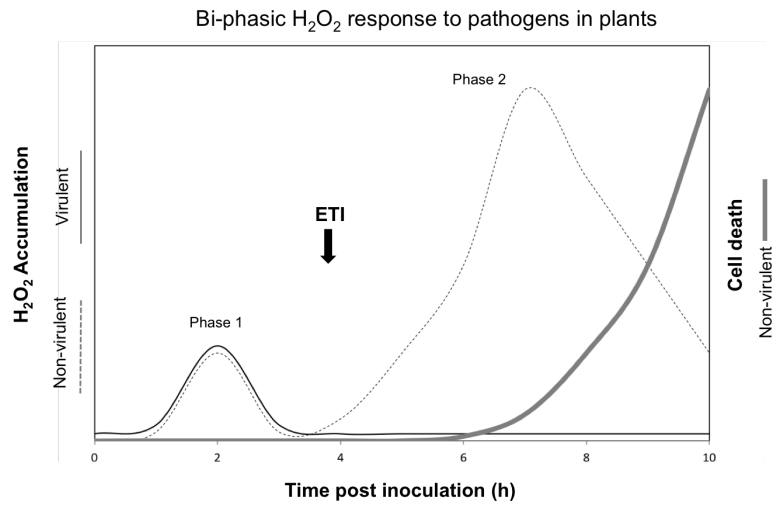

Figure 1.3: Kinetics for accumulation of $\mathrm{H}_{2} \mathrm{O}_{2}$ and induction of cell death in plant cells following bacterial inoculation. Cell death and $\mathrm{H}_{2} \mathrm{O}_{2}$ accumulation following inoculation with non-virulent pathogen or non-host pathogen; $\mathrm{H}_{2} \mathrm{O}_{2}$ accumulation following inoculation with virulent pathogen. The black arrow show the point when the ETI is triggered by a non-virulent invading pathogen causing the phase 2 spike of $\mathrm{H}_{2} \mathrm{O}_{2}$. Reproduced from Lamb and Dixon (1997) [70].

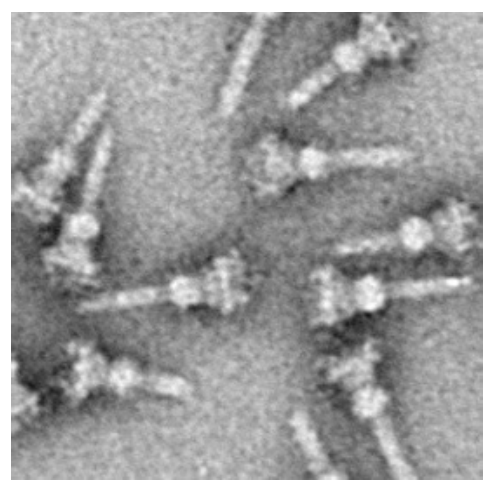

Figure 1.4: Salmonella typhimurium type III secretion needle complex. Schraidt et. al., 2010, [85], Image used under creative commons license.

Injected effectors have a diverse range of activities, they can intercept the PTI signal cascade [86], regulate plant gene transcription, cause nutrient leakage and otherwise promote pathogenicity. However, the biochemical function or targets of most effectors still remain largely unknown $[87,88]$. Early observation found that loss of function mutations in the T3SS caused virulent bacteria to lose pathogenicity and elicit the PTI, which indicated a role for effectors in the PTI suppression. [89-91]. One family of effectors that work to block the PTI have been shown to inhibit a MAPK signalling pathway directly, the activation of which would otherwise lead to the induction of defence mechanisms by dephosphorylating 
key enzymes in the PTI signalling cascade $[92,93]$. Other effectors can inhibit signalling at the pathogen recognition stage, upstream of the MAPK pathway, to block innate immunity [94]. Figure 1.5 depicts an overview of effector delivery into the plant cell cytoplasm and the sites of action within the plant cell where effectors work to block innate immunity. It is ultimately the specific arsenal of effectors carried by a pathogen strain that allows it to tailor virulence towards a specific host. However, these effectors can be a double-edged sword for the pathogen, as they can also prompt the second branch of plant immunity, which endows certain plant cultivars with resistance to particular pathogens.

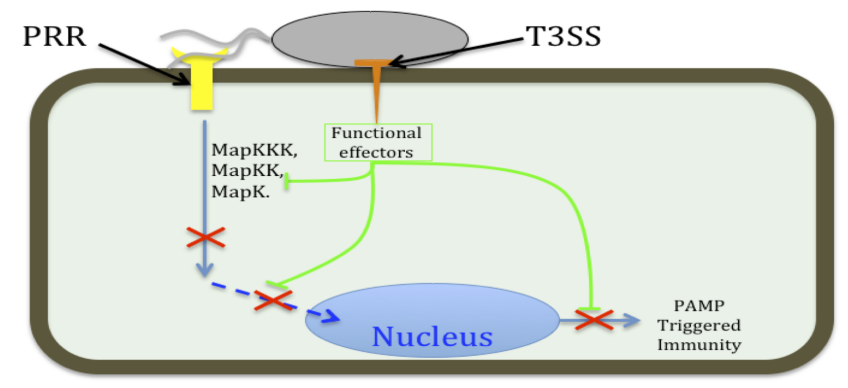

Figure 1.5: Susceptible host-pathogen interaction: Effectors secreted by the T3SS interact at crucial signalling steps to block the immune response.

\subsubsection{Plant resistance to effector mediated attack}

In nature, plants and their pathogens are in a constant genetic attack and defence contest. The results of this contest will determine the outcome of infection [95, 96]. Certain cultivars are able resist a specific effector mediated attack of a particular pathogen by the gene for gene evolution of a cognate resistance $(\mathrm{R})$ protein, which can detect and arrest the pathogen effector [97]. The gene for gene concept refers to the observation that disease resistance in plants is a function of the interaction between the products of a single plant $\mathrm{R}$-gene and a complementary pathogen effector-gene [98]. The plants expressing an effective $\mathrm{R}$ protein will be naturally selected for as they are resistant to the reciprocal pathogen. Resistance to the pathogen will be maintained in the population of plants until the pathogen undergoes some genomic rearrangement or addition and acquires a novel functional effector complement that is functional and unrecognisable to the plant [97]. the pathogen recognition stage

\section{Resistance proteins}

$\mathrm{R}$ proteins act as molecular guards and recognise cognate effectors (or host modification as a result of the effectors). The effector stimulated $R$ protein arrests the effector attack by activating the second stage of plant immunity called effector triggered immunity (ETI). Many $\mathrm{R}$ proteins are characterised by a nucleotide binding domain leucine-rich repeat and signal further defence mechanism through transcription changes [99, 100]. Like PTI, large-scale changes in the host transcriptome are associated with healthy ETI. This transcriptomic response shears much in common with the PTI in terms of the identity of regulated genes, although there can be substantial differences in the speed and amplitude of 
their transcriptional changes as a more potent and possibly damaging is response required[101, 102].

In order to function, an $\mathrm{R}$ protein will either detect the injected effectors directly or detect the interference caused at the target site of the effector and then signal to induce the changes required (figure 1.6) to accelerate and increase the amplitude of the basic immune response. To induce immunity, some $\mathrm{R}$ proteins can be found in the nucleus acting as transcription factors or mediating a response via interaction with specific transcription factors [66, 86, 103]. Others do not require nuclear localisation to induce an ETI-response. Perhaps the most important factor determining the cellular location of an $\mathrm{R}$ protein is that it must reside where it is able to detect its cognate effector. For example, the P. syringae effector protein AvrRpm1 is targeted to the host plasma membrane and acts up stream of MAPK signalling [104, 105]. The $\mathrm{R}$ protein that recognises AvrRpm1 is RPM1, which is also located on the plasma membrane. However, induction of an ETI-response to AvrRpm1 does not require relocalisation of RPM1 from the plasma membrane to the nucleus and the connecting steps are unknown [106].

\section{Hypersensitivity response}

The most apparent and general effect of an activated ETI, compared to a PTI response, is the increased level of the oxidative burst. This burst, in conjunction with a further increase in SA release, leads to the hypersensitivity response (HR). An effective HR ultimately causes local apoptosis of plant cells, possibly halting further colonisation by the bacteria and thereby conferring resistance to a particular effector-conditioned pathogenic assault [100]. The HR is a well-documented characteristic related to ETI, but the reason for and exact molecular events leading to an HR are contentious and beyond the direct interest of this work (reviewed in [107-109]).

Because $\mathrm{R}$ proteins recognise a single cognate effector, the loss of a particular effector by a pathogen can result in a shift of host specificity, which could result in an emergent pathogen. This host shift happens when the $\mathrm{R}$ proteins that would usually give the cultivar resistance are unable to detect a pathogen attack because the cognate effector needed to trigger the $\mathrm{R}$ protein is not present in the emergent pathogen thus the ETI / HR may no longer be induced or at least not induced as strongly by pathogen attack. It would seem likely that a highly virulent emerging pathogen could arise by acquiring a new effector to replace one that has been lost, perhaps a functional homologue to suppress the host's immunity, but one that has a unique amino acid sequence that avoid detection by $\mathrm{R}$ proteins [110].

\subsubsection{Summary / closing remarks on immunity}

Immunity to bacterial pathogens in plant populations is possible and indeed it is necessary for the survival of wild and cultivated species. It is a desirable characteristic that is often selected for when artificial breeding of horticultural crops is carried out. A consensus that the long-term resolution to the Psa-V epidemic will be the development of resistant 


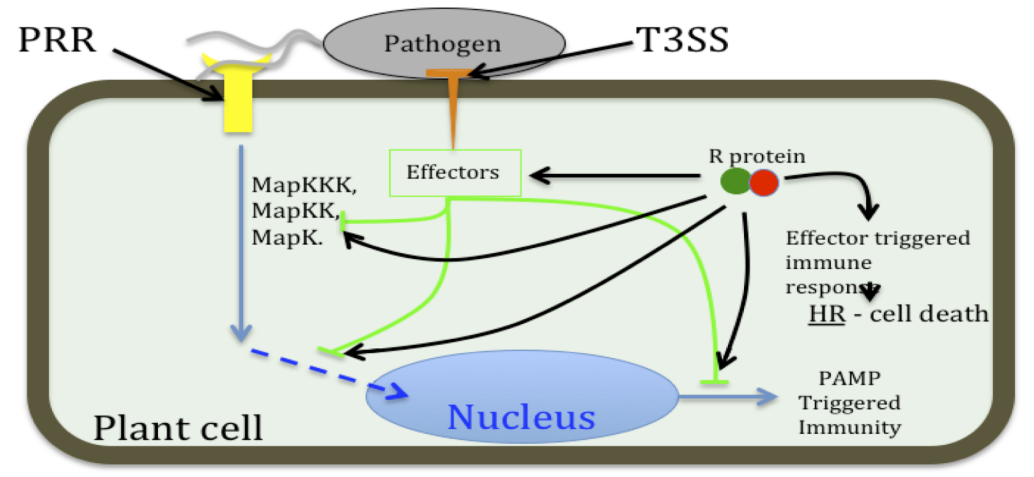

Figure 1.6: Resistant plant pathogen interaction: $\mathrm{R}$ proteins recognise effectors or their interactions with signalling pathways. Once activated, R proteins initiate the ETI and the HR.

kiwifruit cultivars by breeding in resistance is held by Psa-V researches and industry leaders. Cross breeding with resistant germ stock to introduce the required $\mathrm{R}$ gene(s) into plants that have market potential has been the classic approach to produce viable and immune crops. This process is indirect and subject to random genetic recombination, thus the nature of this breeding process requires experimental set ups of long duration and is resource intensive. In the case of Psa-V's sudden and sever impacts interim solutions to control infection are sought, nonetheless breeding Psa-V immune kiwifruit cultivars is an important goal. A molecular approach using the yeast two-hybrid system to elucidate the interactions of an important plant defence proteins, which are known to be effector targets and are required for ETI, is underway in order to guide resistance breading programs. High throughput breeding programs using molecular technologies to fast track immunity out comes are also in progress. Other plant characteristic besides $\mathrm{R}$ genes, such as morphology, physiology and biochemistry differences, can also facilitate increased pathogen tolerance.

In horticulture practices, the plant's basal immunity to microbes has been manipulated with chemical and biological technologies to increase the plants resistance to problematic pathogens, for example synthetic immune elicitors and growth enhancing or protective microbes. The invention and use of similar technologies suitable for kiwifruit cultivation may provide a sustainable interim solution during the process of breeding endogenous immunity into a commercially viable kiwifruit strain.

\subsection{Genetic studies of Psa}

In order to understand how Psa-V can achieve such a high level of destruction, as well as the lack of efficacy of many of the standard control measures, comprehensive genetic studies of Psa-V have been undertaken including complete genome sequencing. These studies are also supporting molecular research strategies to address these issues.

\subsubsection{Genetic studies}

In general for $P$. syringae pathovars the core genomes primarily comprise housekeeping genes and are very stable, with clonal populations of strains emerging worldwide [111, 112] (although it is important to note that this analysis may reflect a sampling bias, due to 
preferential focus on agriculturally significant pathovars [113]). However, additional regions of the genome do exhibit much more variability, in particular the regions that are associated with host-specific virulence [34]. The most important of the host-specific virulence genes encode for the secreted proteins (effectors) that, as described above, modulate the host's induction of antimicrobial immunity [92]. Known virulence factor genes in P. syringae pathovars, for example the type III secretion system (T3SS) effector genes of the exchangeable effector locus, are often situated in pathogenicity islands and are associated with mobile genetic elements [114]. P. syringae is a very genetically promiscuous organism and in response to certain cues, particularly those associated with stress, can induce activation of island excision mechanisms to quickly lose regions of genetic material. Similarly, the rate of gain of new genetic material by horizontal gene transfer is increased in survival conditions found outside the host plant. This can allow the bacteria to acquire genes that improve its chance of survival in a novel environment. Mixed bacterial populations facilitate the flow of new genetic material for this process [115]. The significance of these observations is that Psa- $\mathrm{V}$ would not have had to independently evolve important virulence factors (or for that matter copper / antibiotic resistance), as evolution can take place cooperatively in a bacterial community of many pathovars, species and genera, which all exchange genetic information horizontally [40, 116]. It should also be noted that all Psa/Psa-V strains examined thus far have been found to contain putative phage integrases, integrase family proteins, and transposases [29]. These mobile elements promote rapid evolutionary events and allow the pathogen to extend or change its host range by acquisition or loss of virulence factors, which can in turn contribute to rapid changes in host-pathogen interactions and contribute to the sudden emergence of epidemic strains $[117,118]$. This is especially relevant in the setting of the kiwifruit industry where there is limited host genetic variation due to plant cloning practices and the resulting monoculture. Psa-V and commercial kiwifruit in New Zealand therefore have differing levels of genetic variation and different mechanism for creating genetic variation, resulting in Psa-V having a higher evolutionary potential [34, 112].

\subsubsection{Psa population diversity}

There are at least four genetically distinct populations of $P$. syringae pv. actinidiae that infect kiwifruit plants worldwide [29]. These different strains have varying abilities to cause disease in the host. The range of virulence is likely due to variation in the complements of virulence factors and effectors unique to each strain. Thus, molecular differences between the high and low virulence strains may aid in the identification of targets to help the development of disease control.

\subsubsection{Genome features of Psa}

In an in-depth study comparing the genome sequence data of pre- and post-epidemic populations of $P$. syringae pv. actinidiae alongside data of all other $P$. syringae pathovars available, it was suggested that Psa-V shares a common ancestor with the Psa found in pre-epidemic times, possibly from east Asia. However, it was not thought to be derived 
directly from the pre-2008 Italian strain [34]. The study found that Psa-V has 398 open reading frames (ORFs) that are unique to it when compared to the ORFs of the Japanese and pre-2008 Italian Psa strains. Of these unique ORFs, 49\% have homologs in at least one of the P. syringae pv. tomato genomes that were also assessed. These results suggest some level of horizontal gene transfer from $P$. syringae genomospecies 3 (in which $P$. syringae pv. tomato is placed). Similar comparative studies by a group at Otago report that Psa-V cannot have been derived from the Psa-LV stain which is endemic to New Zealand. The most recent genetic work proposed China as the likely origin for the New Zealand epidemic strain. [20, 30, 119].

\subsubsection{Origins of Psa-V}

An important goal of several recent studies of Psa / Psa-V has been to identify the geographic origin of the virulent strain, with published article titles including: "A multiplex PCR assay for detection of Pseudomonas syringae pv. actinidiae and differentiation of populations with different geographic origin" [120]; "Pseudomonas syringae pv. actinidiae (PSA) isolates from recent bacterial canker of kiwifruit outbreaks belong to the same genetic lineage" [119]; and "Pseudomonas syringae pv. actinidiae from recent outbreaks of kiwifruit bacterial canker belong to different clones that originated in China" [30]. The reasons given for this fixation on the origin of Psa- $\mathrm{V}$ concern a desire to identify the modes of dissemination (especially international spread), prevention of further spread, and to allow a rapid differentiation of strains from diverse origins.

One of these studies, published in May of 2012 by Mazzaglia et al. [119], used a reductionist approach to produce a phylogeny from the genome sequences of $45 \mathrm{P}$. syringae strain, nine of which were Psa genomes sequenced by the authors from isolates originating in either Italy, Portugal, Japan, Korea or China. The remaining strains were other $P$. syringae genomes available on public databases or sequenced in-house by the authors and their collaborators. In addition, data from two P. fluorescens stains were used as out groups. A total of 1,186 protein families were identified that had exactly one member per genome in all the P. syringae and outgroup genomes used. These protein orthologue sequences were aligned and concatenated to reveal a phylogenetic relationship displayed as a maximum likelihood tree. This was the first inclusion of Chinese Psa isolates in an in depth molecular analysis of the pathogen. The phylogeny that was produced suggested at least two lineages of Psa across global populations, with the strains from Korea and Japan separating from the post-2008 strains of Chinese and European origin. This bifurcation of the population was in accordance with previous MLST analysis results [25, 28]. Taking stock of these finding we have the very probable situation where the Korean, Japanese and pre-2008 Italian strains are excluded from the current outbreak strains and the virulent global outbreak is a young lineage of strains that has undergone horizontal gene exchange in areas of the genome known for mobility. The authors tentatively suggest that two isolated events stemming from China led to the incursions in Italy in 2008 and in New Zealand in 2010, based on the SNP similarity found within populations from these geographic locations pointing to a single origin and vast differences between the PIs sequences found in Italian 
and New Zealand isolates pointing to discrete dispersal events. Also credited is the logical pattern of phylogenetic chronology (SNPs/time) observed across the examined populations leading the authors to propose a common ancestor of the Italian and New Zealand strain had existed rather than one being derived from the other.

\subsubsection{Integrative conjugative elements - ICEs}

Initial interpretation of genetic data revealed a feature within Psa-V genomes that resembled the pathogenicity island called PPHGI-1 which is found in P. syringae pv. phaseolicola. Butler et al., (2013) were first to fully describe the island in Psa-V as an integrative conjugative element (ICE) and they showed it actually has more similarity to an island found in P. syringaepv. syringae B728a, denoted as PsyrGI-6 [30]. In total, three different ICE subgroups are recognised in this study and they can be categorised as New Zealand / Chinese (ICE-1), Italian/Chinese (ICE-2), and Chilean (ICE-3). Comparative analysis of this $\sim 100 \mathrm{~kb}$ region in Psa-V from areas with recent outbreaks show they are highly divergent, although broadly syntenic. Interestingly the ICE from the Italian biovar was identical to an ICE found in a strain from China. Furthermore, another ICE from a different Chinese biovar included in the study was $>95 \%$ identical to the ICE found in New Zealand strains and importantly the ICEs in Italian and New Zealand strains are only about $50 \%$ similar and are located at different integration sites [20]. These are the areas that show the most diversity amongst geographic Psa-V isolates and are somewhat informative as to the origins of Psa-V. Although highly divergent, the ICE sequences encode mostly uncorrupted ORFs and the data predicts these are under strong stabilising selection. This suggests the genes are functional and thus not subject to a high rate of random substitution mutations.

Features of the ICE regions were defined and examined [30]. The regions are enclosed by attachment sites (attL and $a t t R$ ) and have numerous sequences predicted to be involved in integration and conjugation. First noted in the analysis are conserved genes needed for ICE function including recombinases and DNA mobility genes, genes that make up the back bone of the ICEs. Other genes contained in the ICEs could affect the phenotype of the pathogen, being genes conserved in all three ICEs and others specific to the geographically diverse ICE subgroups. For example, ICE- 1 of the New Zealand biovar contains a C5 specific DNA methylase while the Italian ICE-2 has three arsenate resistance genes not found in the other ICEs. Many ORFs of unknown function are located in the ICEs as well, e.g. ICE-2 includes a large $(6 \mathrm{~kb}) \mathrm{ORF}$ that has no close resemblance to any annotated gene. Shared by all Psa-V ICEs is the XerC / D transposon - Tn6212. This transposon is located at the same position in all Psa-V ICEs. The first ORF of this autonomously mobile element is a XerC like tyrosine recombinase and this is followed by many other genes possibly influencing phenotype and which may be important in virulence[30].

\subsubsection{Plasmids}

Several studies have made it clear that Psa-V contains a plasmid [20, 34]. The complete genomic assembly of Psa NZ V13 by MacCann et al. (2013) describes a $70.1 \mathrm{~kb}$ 
autonomously replicating plasmid found in this strain. Annotation of the plasmid sequence reveals the presence of genes that encode for effectors as well as two gene clusters involved in the metabolism of aromatic carbon. It is suggested that the plasmid could be important for the infection of vascular tissue.

\subsubsection{Toxin production}

One major difference between pre-epidemic Psa and Psa-V is the lack of an excreted tripeptide derived virulence factor, phaseolotoxin, in Psa-V. Phaseolotoxin is produced by all earlier (pre-2008) isolates of Psa tested, but it is not common among other P. syringae pathovars [117]. It is responsible for causing a chlorotic halo (yellowing) at the sites of infection by pre-2008 Psa strains on kiwifruit leaves and it also induces a strong antimicrobial effect. The effects of phaseolotoxin are not host specific and are due to the inhibition of arginine metabolism. Phaseolotoxin-producing Psa possess the pht biosynthetic gene cluster, a mutant version of ornithine carbamoyltransferase $(\operatorname{argK})$ which confers phaseolotoxin resistance to the bacteria producing the phaseolotoxin, and a non-ribosomal peptide synthase (NRPS) which is also involved in synthesis of phaseolotoxin. All three types of genes are under the control of the GacS / GacA global transcription regulatory system and the pht biosynthetic gene cluster is shown to have been gained by lateral gene transfer[117, 121]. The acquisition of these genes exemplifies the flexibility of the virulence associated genes of $P$. syringae in general.

Although Psa-V does not produce phaseolotoxin, and does not possess the genes required to do so, chlorosis is nonetheless observed at sites of Psa-V infection (see figure 1.7).

Genomic data has revealed that Psa- $\mathrm{V}$ contains a unique genetic region that contains three NRPS modules of unknown function [20]. To date this is the most likely candidate for encoding the putative toxin responsible for the chlorosis generated during Psa-V infection.

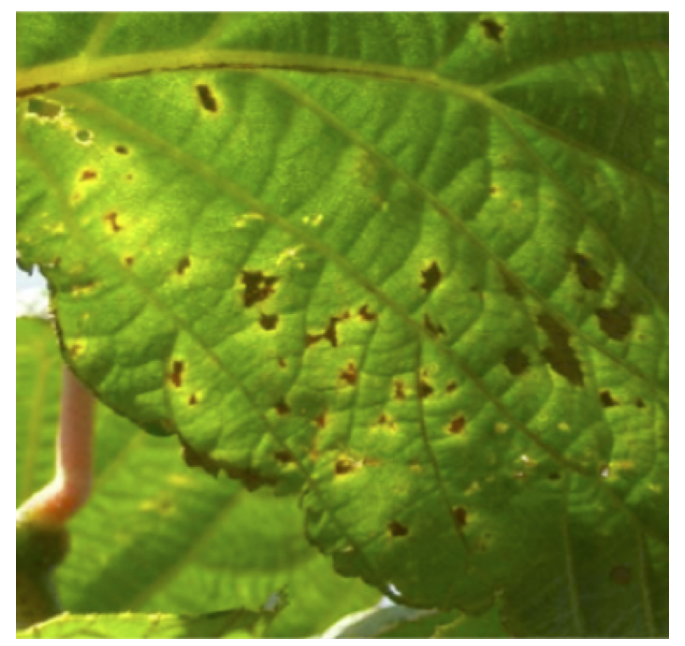

Figure 1.7: Chlorosis: Psa-V infected kiwifruit leaf. Chlorosis (yellowing) can be seen surrounding the angular necrotic regions of infection sites at Te Puke. (Photo credit Zespri)

\subsubsection{Unique effectors}

The comparative genomic studies of Psa/Psa-V have revealed putative virulence factors that are found in the Psa-V genome but are not present in the less virulent Psa strains. 
These are of particular interest to researchers, as unique or exclusive combinations of effectors may be making key contributions to the heightened virulence of Psa-V. Of particular note, while there is a core set of 40 known effector proteins shared between Psa and Psa-V, Psa contains a subset of four effector genes not represented in Psa-V, whereas Psa-V contains four effectors that are not found in any of the lower virulence strains of Psa. The four unique effectors found in Psa-V are hopA1, hopH1, hopAA1-2 and hopZ5 (previous labelled hopZ2-like or hopZ2b) [20, 34, 122]. Of these four, hopZ5 is the most unique effector in Psa- $\mathrm{V}$, sharing only $40 \%$ identity with the closest known orthologue in any species, and it is considered an entirely new effector gene [Dr. Matt Templeton, personal communication]. The hopA1 and hopAA1-2 effectors are rare in P. syringae and previously only found together in three $P$. syringae pv. tomato strains out of the 91 strains of P. syringae examined [123]. As discussed above in section 1.5.3, the loss or lack of one or more effectors can sometimes result in renewed or heightened virulency. Such differences may hold the key to unlocking the virulence of Psa-V but yet have not been thoroughly investigated. Nonetheless, targeting of Psa-V specific virulence factors may be useful in developing control agents and any of the unique factors above could potentially be a target if found to contribute to the disease phenotype of Psa-V. Possible targets of relevance to this study are discussed in more detail in section 4.2 .

\subsection{Study focus}

\subsubsection{Study objectives}

The overall goal of this study was to investigate or develop microbial control options that could assist kiwifruit growers in New Zealand in reducing the incidence of infection and disease symptoms on kiwifruit vines due to the recently introduced pathogen $P$. syringae pv. actinidiae. During this study two separate threads of research were pursued. First, in response to the most immediate interest from the kiwifruit cooperative co-funding this work (Seeka Kiwifruit Ltd.), a Psa-V antimicrobial assay was optimised and implemented. The aim of this section of work was to provide an accurate evaluation of the relative antimicrobial effect of various antibiotics and other known or suspected antimicrobial compounds against Psa-V, to support development of possible orchard spray regimes or for sterilisation of orchard equipment. Second was the development of a platform for targeted knockout of virulence genes in Psa-V, which ultimately constituted the major focus of this study. The objective of this focus was to produce knock-out strains to be investigated as antagonistic biocontrol agents and/or research tools to understand the pathogenesis of Psa-V.

\subsubsection{Considerations}

Several strains of Psa / Psa-V were originally sent from the International Collection of Microorganisms from Plants (ICMP), Landcare Research (under Biosecurity New Zealand Chief Technical Officer (CTO) approval), to the Ackerley lab at the commencement of this study. Chief among these strains was P. syringae pv. actinidiae ICMP 18800, selected to be 
the principal strain used throughout this study. This strain was selected on the basis of it displaying the high virulence phenotype and its site of geographical isolation near the original outbreak in New Zealand. This was also one of two strains initially selected by our genome sequencing collaborators at Otago University, and the draft genome sequence data was made progressively available throughout the course of this study. Psa-V NZ 13 was the final strain from which genetic data was accessed because it was the most complete and annotated data set compiled for Psa-V. This strain was also supplied to me for use in laboratory experiments but the isolate had a shallow growth curve so ICMP18800 was routinely employed for experimental work. A draft data set for ICMP 18800 was made accessible to me in the earliest stages of this study. Later on during the course of this study another genome sequenced strain was generously provided by Dr Matt Templeton (Plant and Food Research Ltd.) and tested in some assays; however, for continuity, the strain ICMP 18800 was the primary focus throughout this thesis. Unless stated otherwise, it can be assumed that this is the strain referred to in each Results chapter.

The Biosecurity New Zealand CTO approval enabled the organisms to be imported into the Ackerley lab and this clearance was sufficient to cover the antimicrobial experiments aspects of this study. However, further Environmental Protection Authority approval was required to carry out genetic modification of this Category 2 host organism (as per the HSNO - Low Risk Genetic Modification Regulations). This approval was obtained via the rapid assessment, low-risk pathway of the Environmental Protection Authority.

\subsection{Aims}

\subsubsection{Design and optimise an assay to rapidly test a range of putative or known antimicrobial agents and quantify their efficacy against Psa-V.}

A key goal of this assay was that it should produce data suitable to calculate quantitative $\mathrm{IC}_{50}$ values that could be used to guide antimicrobial concentrations in field trials and monitor for the emergence of fitness differences in suspected or known resistance strains. Should ideally be established in a 96 well-plate format to allow serial dilutions to be easily prepared and take advantage of our high throughput plate reader.

\subsubsection{Develop a platform for producing in-frame gene knockouts of Psa-V based on a strategy already proven effective in $P$. syringae pv. phaseolicola 1448a.}

This system is based on a two-step integration and excision mutagenesis protocol, employing suicide plasmids containing both selectable and counter-selectable markers. Pseudomonas species and strains are known to vary dramatically in their genetic tractability (i.e. the ease with which targeted mutations and recombination events can be generated therein), because of efficient endonuclease systems, and thus it was anticipated that substantial optimization might be required to instigate this platform (Dr David Ackerley, personal communication). Once optimised, the mutagenesis protocol will be 
applied to wild type Psa-V to knockout promising gene targets identified by analysis of emerging Psa-V sequence data. 


\title{
Chapter 2
}

\author{
Materials and Methods
}

\subsection{Chemicals, media, enzymes and reagents supplies.}

The chemicals used to prepare all buffers, reagents, antibiotics and media used in this study were of analytical grade and obtained from either Sigma Aldrich (St. Louis. MO, USA) or Biolabs and Ajax Finechem, both part of Thermo-Fisher Scientific (Waltham, MA, USA). The LB growth media used for the maintenance of bacterial cultures was from either Sigma Aldrich or Duchefa (The Netherlands). Restriction enzymes were supplied by New England Biolabs (NEB; Ipswich, MA, USA). The T4 DNA ligase used was supplied by Invitrogen (Life Technologies; Carlsbad, CA, USA). BioMix TM Red was supplied by Bioline (London, $\mathrm{UK})$.

\subsection{Bacterial strains, plasmids and primers.}

\subsubsection{Bacterial Strains}

Strains of Escherichia coli, P. syringae and P. aeruginosa used throughout this study are presented in the following tables.

Table 2.1: E. coli strains

\begin{tabular}{lll}
\hline Strain & Relevant Characteristics $\backslash$ Description & Source \\
\hline BL21 & $\begin{array}{l}\text { F- ompT gal dcm lon hsdSb (rb- mb-) (DE3 [lacI lacUV5-T7 gene } \\
\text { BBB 1 ind1 sam7 nin5]). }\end{array}$ & Lab stock \\
\hline DH5 $\alpha \lambda$-pir & $\begin{array}{l}\text { supE44 DlacU169 (Ø80 lacZ DM5) hsdR17 } \lambda \text { (pir) Used for cloning, } \\
\text { plasmid maintenance and as a host strain in tri-parental conjugation. }\end{array}$ & Lab Stock \\
\hline ANS98 & Tri-parental conjugation helper strain. & Lab Stock \\
\hline S17-1 & $\begin{array}{l}\text { recA pro hsdR RP4-2-Tc::Mu-Km::Tn7 integrated into the chromo- } \\
\text { some, devoid of the E. coli K-12-specific restriction system allowing }\end{array}$ & Prof. Iain Lamont \\
& efficient uptake of foreign DNA. Conjugative transformation host. & \\
\hline WT 3110 & Lab strain. & Lab Stock \\
\hline
\end{tabular}


Table 2.2: $P$. syringae and $P$. aeruginosa strains

\begin{tabular}{lll}
\hline Strain & Relevant Characteristics $\backslash$ Description & Source \\
\hline Psa-V ICMP 18800 & $\begin{array}{l}\text { Pseudomonas syringae pv.actinidiae, Wild type strain, } \\
\text { highly virulent bacterial canker of kiwifruit }\end{array}$ & Landcare Research \\
\hline PsaV-NZ13 & Virulent Psa-V clone, complete sequence published. & Dr. Matt Templeton \\
\hline Psa-V Strep ${ }^{\mathrm{R}}$ & Streptomycin resistant Psa-V derived from ICMP 18800. & This study \\
\hline Psp 1448a & P. syringae pv phaseolicola 1448A, Wild type strain & Lab Stock \\
\hline PAO1 & Pseudomonas aeruginosa PAO1, Wild type strain. & Lab Stock \\
\hline
\end{tabular}

\subsubsection{Plasmids}

Table 2.3: Plasmids used in this study

\begin{tabular}{|c|c|c|}
\hline Plasmid & Description & Source \\
\hline pEX18-GM & $\begin{array}{l}\text { Integrating, suicide knockout plasmid previously used for } \\
\text { gene deletion in } P \text {. aeruginosa and } P \text {. syringae, } \mathrm{Gm}^{\mathrm{R}} \text {, } \\
\text { sacB } B^{+} \text {, ori } T^{+}, \text {lacZa } \text {, MCS from pUC18. }\end{array}$ & Lab stock \\
\hline pEX18-GM::tGacA 2kb & pEX18-GM + Truncated $2 \mathrm{~kb} g a c A$ fragment & This Study \\
\hline pEX18-GM::tGacS 2kb & pEX18-GM + Truncated 2kb gacS fragment & This Study \\
\hline pEX18-GM::t3004 & pEX18-GM + Truncated 2kb 3004 fragment & This study \\
\hline pEX18-GM::t3170 & pEX18-GM + Truncated $2 \mathrm{~kb} 3170$ fragment & This study \\
\hline pEX18-GM::tN1 & $\begin{array}{l}\text { pEX18-GM }+ \text { Truncated } 2 \mathrm{~kb} \text { amino acid adenylation do- } \\
\text { main protein } 1\end{array}$ & This study \\
\hline pEX18-GM::tN2 & $\begin{array}{l}\text { pEX18-GM }+ \text { Truncated } 2 \mathrm{~kb} \text { amino acid adenylation do- } \\
\text { main protein } 2\end{array}$ & This study \\
\hline pEX18-GM::hopH1 & pEX18-GM + Truncated 2kb hopH1 fragment & This study \\
\hline pEX18-GM::hrpA & pEX18-GM + Truncated 2kb hrpA fragment & This study \\
\hline pEX18-GM::hrpK & pEX18-GM + Truncated 2kb hrpK fragment & This study \\
\hline pEX18-GM::hrpL & pEX18-GM + Truncated $2 \mathrm{~kb} h r p L$ fragment & This study \\
\hline pUCP22 & Broad host range plasmid, Ori1600, $\mathrm{Gm}^{\mathrm{R}}$ & Lab stock \\
\hline pSX & $\begin{array}{l}\text { pUCP22 backbone with expression region from pUCX, } \\
\text { allows IPTG regulated expression of His-Tagged proteins } \\
\text { in Pseudomonas species [124] }\end{array}$ & Lab Stock \\
\hline pSX::NRPS2 mod1 & $\begin{array}{l}\text { pSX with the first module of the amino acid adenylation } \\
\text { domain from NRPS2 of Psa-V }\end{array}$ & This study \\
\hline $\mathrm{pSX}:: \mathrm{Kn}^{\mathrm{R}}$ & $\begin{array}{l}\text { pSX with Tn5 neomycin phosphotransferase insert from } \\
\text { pKD4 }\end{array}$ & This study \\
\hline pKD46 & Red recombinase plasmid & Lab stock \\
\hline pKD4 & FRT-Kan-FRT template plasmid & Lab stock \\
\hline pMP190::pvdE & Broad host range IncQ expression vector with $p v d E$ insert & Prof. Iain Lamont \\
\hline pDM4 & Suicide knockout plasmid $\mathrm{Chl}^{\mathrm{R}}, s a c B$ & Lab stock \\
\hline
\end{tabular}




\subsubsection{Primers}

Primers were designed using Geneious R6 bioinformatic software as a platform for visualising genomic data, the final primer designs were selected without the aid of the software's primer design feature, but instead were selected precisely based on the required primer binding positions. Design of Psa-V primers were based on sequence data kindly supplied by Assoc. Prof. Russell Poulter from department of biochemistry at University of Otago and Assoc. Prof. Matt Templeton of Plant and Food research. PsaF1/PsaR2 primers [27], for verification of Pseudomonas syringae pv. actinidiae, were supplied combined at a concentration of $10 \mu \mathrm{M}$ each courtesy of John Mackay. AcsA knockout primers were from lab stocks of a previous study [125]. New primers were ordered from and synthesised by Integrated DNA Technologies (www.idtdna.com). Lyophilized oligonucleotides were reconstituted to a final concentration of $100 \mathrm{\mu M}$ in TE buffer for storage at $-20^{\circ} \mathrm{C}$. A working stock of $10 \mu \mathrm{M}$ was prepared by dilution in filter sterilized $\mathrm{ddH}_{2} \mathrm{O}$. 
Table 2.4: Primers used in this study

Restriction sites are indicated in bold, the homology regions for splicing by overlap PCR are underlined.

\begin{tabular}{|c|c|}
\hline Primer name & $5^{\prime}-3^{\prime}$ sequence \\
\hline \multicolumn{2}{|l|}{ Overlap fragments primers } \\
\hline 3004_Lup_1kb_XhoI & GGGGCTCGAGTCGATGTCGCCGGTACCTG \\
\hline 3004_Ldwn_hom & GCATGCTGTAAGTCAGGAGGCAACAGTCATCGCAAATCC \\
\hline 3004_Rup & $\begin{array}{l}\text { TTA } \\
\text { GCCTCCTGACTTACAGCATGCGCTTGAGGG }\end{array}$ \\
\hline 3004_Rdwn_1kb_XbaI & GGGGTCTAGATACTGCGAAATCGTCGGCCA \\
\hline 3170_Lup_1kb_XhoI & GGGCTCGAGATGATGTCGTCGCCGCTGGCCATCT \\
\hline 3170_Ldwn & GTTCGGCATGAGAAGGTTCCATGGC \\
\hline 3170_Rup_hom & $\begin{array}{l}\text { GGAACCTTCTCATGCCGAACAGCCGAATAATTCGGCGCG } \\
\text { GACCGA }\end{array}$ \\
\hline 3170_Rdwn_1kb_XbaI & GGGTCTAGAGCGCAGGGCCTCGAATTGCGGCGCT \\
\hline gacA_Lup_1kb_XhoI & GGGGCTCGAGCAAACACTGCGAGTGAGCGG \\
\hline gacA_Ldwn & CTTAATCAAGCAGACACCTC \\
\hline gacA_Rup_hom & TGCGCGAGGTGTGATTAAGAGCGCCTGAACATGACCCAG \\
\hline gacA_Rdwn_1kb_XbaI & GGGGTCTAGACGTTGACGACGTTGACGATG \\
\hline gacS_Lup_1kb_XhoI & GGGGCTCGAGTCGGTCTGCCCCATGTCGAT \\
\hline gacS_Ldwn & GGTCAGCACGCTTTTTCTCC \\
\hline gacS_Rup_hom & $\begin{array}{l}\text { TTGCTCGAGAAAAAGCGTGCTGACCACGGCGTGATGAAG } \\
\text { CCTCTC }\end{array}$ \\
\hline gacS_Rdwn_1kb_XbaI & GGGGGTCTAGAACGGCATGCGACGCGCAGCG \\
\hline hrpA_L_up_1kb_SalI & GGGGTCGACGAGTCTTGATGAAAGGTTTGAGGATGATC \\
\hline hrpA_L_dwn & TGCGACCATGATTTGATGCCCCTTAAGA \\
\hline hrpA_Rup_hom & $\begin{array}{l}\text { TAAGGGGCATCAAATCATGGTCGCAAGTTACTAATTATTT } \\
\text { CTGATTGCCCCCTCATCAG }\end{array}$ \\
\hline hrpA_Rdwn_1kb_BamHI & GGGGGATCCCAACTGACCCAGGTCCTGATTGGAC \\
\hline hrpK_Lup_1kb_XhoI & GGGCTCGAGGGCGAAGTCCCTGAATTGTGCAGAT \\
\hline hrpK_Ldwn & TATACGCATGGTGAGTTGCCATCCGA \\
\hline hrpK_Rup_hom & $\frac{\text { CGGATGGCAACTCACCATGCGTATATTCGCCTGAATGGC }}{\text { GATCTCGATCA }}$ \\
\hline hrpK_Rdwn_1kb_XbaI & GGGTCTAGAAGCGCTCCGGACAACCCTGG \\
\hline hrpL_Lup_1kb_XhoI & GGGCTCGAGCCGTGTTGAGGCCGTGCATCAG \\
\hline hrpL_Ldwn & CTGAAACATGGGCTTACCCTGATTTAATGG \\
\hline hrpL_Rup_hom & $\begin{array}{l}\text { AAATCAGGGTAAGCCCATGTTTCAGTTCGCCTGAATGGC } \\
\text { GATCTCGATCA }\end{array}$ \\
\hline hrpL_Rdwn_1kb_XbaI & GGGTCTAGAAGCGCTCCGGACAACCCTGG \\
\hline hopZ2b_Lup_1kb_SalI & GGGGTCGACAGGGACTGGTTCGAGGTTTTTTTGA \\
\hline hopZ2b_Ldwn & AAGTCCCATGGCATGAATCCTCAATGAAATTT \\
\hline hopZ2b_Rup_hom & ATTGAGGATTCATGCCATGGGACTTGAATCCTAAAAGCA \\
\hline & AAGCACTTGGTACCGAAGCT \\
\hline hopZ2b_Rdwn_1kb_XbaI & GGGTCTAGACCTAAGCTCCTCTTTGCCAGATCC \\
\hline
\end{tabular}


Primers used in this study continued

\begin{tabular}{|c|c|}
\hline Primer name & $5^{\prime}-3^{\prime}$ sequence \\
\hline hopH1_Lup_1kb_SalI & GGGTCGACTTAATCATTGAGTCTGCGCTGGCGTATGG \\
\hline hopH1_Ldwn & AGTGATCATAGCTATCCTGAAAAGTTAATTGAGTTG \\
\hline hopH1_Rup_hom & ACTTTTCAGGATAGCTATGATCACTCATCAATAGGGCAACC \\
\hline & TACTGTAAGTTGTTTTTTAG \\
\hline hopH1_Rdwn_1kb_BamHI & GGGGGATCCGCGGGCTGGCCTATAATCTGCATTATC \\
\hline N1_Lup_1kb_XhoI & GGGCTCGAGGTTCTCCTGAGTTTTACCAAAACTG \\
\hline N1_Ldwn & GCTGTTCATTACAGCCCCCCGACCG \\
\hline N1_Rup_hom & $\begin{array}{l}\text { GGGGGGCTGTAATGAACAGCGAACAATGATCGCCGGGCA } \\
\text { GCTGGC }\end{array}$ \\
\hline N1_Rdwn_1kb_BamHI & GGGGGATCCGGACAGTGCCTGGGTAAGCGCGGTA \\
\hline N2_Lup_1kb_XhoI & GGGCTCGAGCCCGGGCCACCGACCCGCGCCGCCG \\
\hline N2_Ldwn & GATACCCATTCGATACACCATCCAT \\
\hline N2_Rup_hom & $\begin{array}{l}\text { TGGTGTATCGAATGGGTATCGCTCGTTGATGTTTACCTACC } \\
\text { ACTC }\end{array}$ \\
\hline N2_Rdwn_1kb_XbaI & GGGTCTAGAATCTCATCAACGCCTGCTGCATGGC \\
\hline acsA_Lup_Xho1 & GGGGCTCGAGATGAACTTCACTTCACTCGCCG \\
\hline acsA_Ldwn & CTCCAGAAACAGCGCTATGCC \\
\hline acsA_Rup_hom & $\begin{array}{l}\text { AAAGGGCATAGCGCTGTTTCTGGAGTGGCTTGAGCGTAA } \\
\text { CGGCA }\end{array}$ \\
\hline acsA_Rdwn_Xba1 & GGGGTCTAGATCAGGTTGCACGGCGCAA \\
\hline \multicolumn{2}{|l|}{$\underline{\text { PSA Primers }}$} \\
\hline PsaF1 & TTTTGCTTTGCACACCCGATTTT \\
\hline PsaR2 & CACGCACCCTTCAATCAGGATG \\
\hline Psa hopZ5 Fwd & ATGGGACTTTGTGCATCAAAACC \\
\hline Psa hopZ5 Rev & TTAGGATTCTATCGCTTTTCTTATTTTTCGT \\
\hline rpsL Fwd & TGACCGAAGCGTTGAACTCCAGCGG \\
\hline rpsL Rev & CTTCTTGGCATGGATAAGCCCTCAAACG \\
\hline PsaNRPS2 m1 Fwd & CCCCATATGAACAGCACCTTTGAAATAACTAATTATG \\
\hline PsaNRPS2 m1 Rev & CCCGTCGACTCAGGCAACCAACGCCATAC \\
\hline \multicolumn{2}{|l|}{$\underline{\text { Other }}$} \\
\hline Kan NdeI Fwd & GGGCATATGATTGAACAAGATGGATTGCACGCAGG \\
\hline Kan Rev SalI & GGGGTCGACTCAGAAGAACTCGTCAAGAAGGCGATAGAA \\
\hline
\end{tabular}

\subsection{Bacterial growth and maintenance}

\subsubsection{Bacterial growth media.}

Unless otherwise noted all media components were dissolved in $\mathrm{ddH}_{2} \mathrm{O}$ and sterilized by autoclaving.

\section{LB}

Rich broth used for the routine growth and maintenance of E. coli and P. syringae cultures. LB was obtained as a premixed powder and when reconstituted it contained: 5 
$\mathrm{g} / \mathrm{L}$ yeast extract, $10 \mathrm{~g} / \mathrm{L}$ bacto tryptone, $10 \mathrm{~g} / \mathrm{L} \mathrm{NaCl}$. Sterilized LB was stored at room temperature.

\section{King's B medium}

An iron limited broth for culturing Pseudomonas spp. This media was made by combining: $20 \mathrm{~g} / \mathrm{L}$ bacto peptone (DIFCO), $1 \% \mathrm{v} / \mathrm{v}$ glycerol, $1.5 \mathrm{~g} / \mathrm{L} \mathrm{K}_{2} \mathrm{HPO}_{4}$. The broth was made up to have a final concentration of $6.1 \mathrm{mM} \mathrm{MgSO}_{4}$. For this, a sterile $1 \mathrm{M}$ stock of $\mathrm{MgSO}_{4}$ was added to the broth after autoclaving to avoid precipitation.

\section{LB Agar}

Prior to autoclaving, agar power was added to LB media at a concentration of $1 \% \mathrm{w} / \mathrm{v}$ to act as a setting agent, producing a solid growth media. Approximately $25 \mathrm{~mL}$ of sterilized and hot liquefied agar containing media was poured into sterile $90 \mathrm{~mm}$ Petri dishes, protected from contamination in a class 2 biological safety hood, and allowed to cool and solidify. These plates were refrigerated at $4{ }^{\circ} \mathrm{C}$ for long-term storage and left at room temperature for short-term storage of less than one week.

\section{Psa selective Agar.}

Unknown composition supplied by Fort Richard Laboratories. Selection based on casein hydrolysis and antibiotic resistance inherent to Psa-V (Auckland, New Zealand). Stored at $4{ }^{\circ} \mathrm{C}$.

\subsubsection{Media supplements}

IPTG (isopropyl -beta-D-thiogalactoside) stocks were prepared to a final concentration of 0.1 M. Xgal (5-bromo-4-chloro-3-indolyl--D-galactopyranoside) stocks were prepared to a final concentration of $40 \mathrm{mg} / \mathrm{mL}$ in 100\% DMSO. All antibiotic stocks were made to $1000 \mathrm{X}$ the media concentration indicated for $E$. coli and dissolved in $\mathrm{dd}_{2} \mathrm{O}$ with the following exception; chloramphenicol (Chl) was dissolved in $80 \%$ ethanol. Supplements were added post autoclaving once the media had cooled to $<65{ }^{\circ} \mathrm{C}$. For premade PSA selective agar, antibiotics solutions were surface spread directly on to the agar and allowed to dry in a biological safety hood. Nitrofurantoin was supplemented at $50 \mathrm{\mu g} / \mathrm{mL}$ into KB or LB plates to select for Psa-V against E. coli. For SacB selection, LB supplemented with 5\% or $10 \%$ $\mathrm{w} / \mathrm{v}$ sucrose was prepared by dissolving the required amount of sucrose in the smallest volume of water possible and the filter sterilized using a $0.22 \mu \mathrm{m}$ pore membrane filter. Sterile sucrose solution was then added to the required volume of autoclaved LB to achieve the desired concentration. Unless otherwise noted, the final media concentration of antibiotics was as listed for each organism in table 2.5. 
Table 2.5: Antibiotics used in this study for cloning

\begin{tabular}{llll}
\hline Antibiotic & E.coli $(\mu \mathrm{g} / \mathrm{mL})$ & Psa-V $(\mu \mathrm{g} / \mathrm{mL})$ & P.s 1448a $(\mu \mathrm{g} / \mathrm{mL})$ \\
\hline Chloramphenicol (Chl) & 34 & 34 & 34 \\
Gentamicin (Gm) & 12.5 & 20 & - \\
Ampicillin (Amp) & 100 & 100 & - \\
Kanamycin (Kan) & 50 & 50 & - \\
Streptomycin (Strep) & - & 50 & - \\
\hline
\end{tabular}

\subsubsection{Bacterial growth conditions}

LB was used for routine growth and maintenance of all bacterial strains. Growth phase was determined by reading the OD600 of $1 \mathrm{~mL}$ of culture. Growth temperatures used were typically: $37{ }^{\circ} \mathrm{C}$ for E. coli and $28^{\circ} \mathrm{C}$ for both P. syringae strains. For liquid cultures aeration was provided by shaking at $200 \mathrm{rpm}$. Medium-term maintenance was achieved by storing agar plates containing bacteria at either $4{ }^{\circ} \mathrm{C}$ when possible or at room temperature for up to a week. For long-term maintenance of strains, an aliquot of overnight culture was mixed 1:1 with $80 \%$ glycerol and stored at $-80{ }^{\circ} \mathrm{C}$. To ensure maintenance of plasmids with antibiotic resistance markers, the appropriate antibiotics were added to liquid and solid media.

\subsection{Routine molecular biology}

\subsubsection{PCR protocols}

BioMix Red was used for overlap PCR mutagenic fragment generation and colony PCR for recombinant clone selection and strain verification.

\subsubsection{Standard BioMix Red ${ }^{T M}$ PCR}

This standard PCR protocol was used to amplify genes for cloning, generate truncated gene fragments for overlap PCR and to serve as a verification tool of plasmid constructs. This protocol was also used as the template for the colony PCR protocol. 


\section{Standard BioMix Red ${ }^{T M}$ PCR components:}

Table 2.6: Standard BioMix Red ${ }^{T M}$ PCR components

\begin{tabular}{|c|c|}
\hline Reagent & Per $20 \mu L$ \\
\hline 2X BioMix $\operatorname{Red}^{\top M}$ (composition is proprietary information) & $10 \mu \mathrm{L}$ \\
\hline Primer $1(10 \mu \mathrm{M})$ & $1 \mu \mathrm{L}$ \\
\hline Primer $2(10 \mu \mathrm{M})$ & $1 \mu \mathrm{L}$ \\
\hline DMSO & $0.5 \mu \mathrm{L}$ \\
\hline Template gDNA & $0.5 \mu \mathrm{L}$ \\
\hline ultra-pure water & $7 \mu \mathrm{L}$ \\
\hline
\end{tabular}

Standard BioMix Red ${ }^{T M}$ PCR thermocycler protocol:

Table 2.7: Standard BioMix Red ${ }^{\mathrm{TM}}$ PCR thermocycler protocol

\begin{tabular}{lll}
\hline Temperature & Time & Cycles \\
\hline $\begin{array}{l}\text { Initial denaturation } \\
95{ }^{\circ} \mathrm{C}\end{array}$ & $5 \mathrm{~min}$ & 1 cycle \\
\hline $95{ }^{\circ} \mathrm{C}$ & $30 \mathrm{~s}$ & \\
$58{ }^{\circ} \mathrm{C}$ & $30 \mathrm{~s}$ & 10 cycles \\
$72{ }^{\circ} \mathrm{C}$ & $1 \mathrm{~min} / \mathrm{kb}$ & \\
\hline $95{ }^{\circ} \mathrm{C}$ & $30 \mathrm{~s}$ & \\
$54{ }^{\circ} \mathrm{C}$ & $30 \mathrm{~s}$ & 20 cycles \\
$72{ }^{\circ} \mathrm{C}$ & $1 \mathrm{~min} / \mathrm{kb}$ & \\
\hline Final extension & & \\
$72{ }^{\circ} \mathrm{C}$ & $10 \mathrm{~min}$ & 1 cycle \\
$14{ }^{\circ} \mathrm{C}$ & Hold & \\
\hline
\end{tabular}

\subsection{DNA extraction purification and manipulation.}

\section{Genomic DNA Extraction}

Bacterial genomic DNA was extracted from $3 \mathrm{~mL}$ overnight cultures using the DNeasy Blood \& Tissue kit (Qiagen GmbH, Germany), following the manufacturers instructions. Genomic DNA preparations were stored at $-20^{\circ} \mathrm{C}$.

\section{Midi Prep Plasmid isolation}

When high concentrations of plasmid DNA (up to $1 \mu \mathrm{g} / \mu \mathrm{L}$ ) were required the Qiagen CompactPrep Plasmid Midi core kit was used as per the manufacturer's instructions from $50 \mathrm{~mL}$ of culture grown in LB supplemented with antibiotic. 


\section{PCR clean up}

Zymo DNA clean and concentrator (Zymo Research, Irvine, CA, U.S.A.) spin columns were used to prepare PCR products for downstream applications. Supplied binding buffer and wash buffer were used. To elute the product $60{ }^{\circ} \mathrm{C}-70{ }^{\circ} \mathrm{C}$ ultra-pure water was used.

\subsubsection{Tris Acetate-EDTA (TAE) agarose gel electrophoresis}

DNA electrophoresis was performed in TAE buffer. The buffer was prepared as a $50 \mathrm{X}$ stock solution and then diluted with deionised water to a $1 \mathrm{X}$ working solution when used to prepare electrophoresis running buffer and gels. The 50X stock solution was made by dissolving $242 \mathrm{~g}$ Tris base $(\mathrm{FW}=121.14)$ in approximately $750 \mathrm{~mL}$ deionized water. Added to this was $57.1 \mathrm{ml}$ glacial acetic and $100 \mathrm{~mL}$ of $0.5 \mathrm{M}$ EDTA (pH 8.0) the solution was adjusted to a final volume of $1 \mathrm{~L}$ with deionized water.

Table 2.8: Tris Acetate-EDTA buffer

\begin{tabular}{ll}
\hline Component & Final 1X concentration \\
\hline $40 \mathrm{mM}$ Tris acetate & $40 \mathrm{mM}$ \\
EDTA & $1 \mathrm{mM}$ \\
\hline
\end{tabular}

\section{Agarose gel extraction}

Zymo DNA clean and concentrator spin columns used in conjunction with ADB was employed to extract electrophoresed DNA from 0.8\% TAE agarose gels. Up to $100 \mu \mathrm{L}$ of PCR product was used. The required sized band was excised from the agarose gel and dissolved in excess $\mathrm{ADB}$ at $65{ }^{\circ} \mathrm{C}$ for 15 -30 mins with occasional vortexing. The entire resulting solution was then passed through a Zymo spin column $600 \mu \mathrm{L}$ at a time by centrifugation. The column was then washed and eluted as per manufacturer's instructions.

\section{DNA quantification}

Nanodrop: To determine DNA concentrations and purity after extraction, isolation and purification the Nanodrop ND-1000 Spectrophotometer was used with $1 \mu \mathrm{L}$ of the sample preparation.

Agarose gel electrophoresis: To analyse the size of PCR products, plasmid digests and other DNA samples, 5-10 $\mathrm{LL}$ samples were run on ethidium bromide-containing TAE agarose gels $(1 \% \mathrm{w} / \mathrm{v}$ agarose in $1 \mathrm{X}$ TAE buffer, $1 \mathrm{\mu g} / \mathrm{mL}$ ethidium bromide), submerged in 1X TAE buffer in a suitable electrophoresis tank at 120 to $140 \mathrm{~V}$ for 20 to $40 \mathrm{~min}$. HyperLadder I (Bioline) was run alongside samples for size comparison. DNA bands were viewed and photographed under ultra-violet (UV) light.

\section{Restriction digests}

NEB restriction enzymes were used to digest plasmids and DNA insert fragments. Insert DNA digests were carried out according to the manufacturer's directions. Plasmid digests 
were optimized, to reduce uncut plasmid background, by making the following changes. Plasmid DNA heated to $65{ }^{\circ} \mathrm{C}$ and cooled to $37{ }^{\circ} \mathrm{C}$ before being added to the digest reaction. After $\sim 16$ hours incubation at $37^{\circ} \mathrm{C}$ an additional $10 \mathrm{U}$ of restriction enzymes are added to the reaction and returned to $37^{\circ} \mathrm{C}$ incubation for 1 hour. Digest reactions were terminated by heat inactivation at $65{ }^{\circ} \mathrm{C}$ for 20 mins.

\section{Ligations}

Ligations reaction were set up using a 1:6 molar ratio of vector to insert ratio with $100 \mathrm{ng}$ of vector per ligation in a total volume of $100 \mu \mathrm{L} .1 \mathrm{U}$ of T4 DNA ligase was used per reaction. Reactions were buffered at recommended concentration with supplied buffer and incubated at $16{ }^{\circ} \mathrm{C}$ overnight. Ligation products were immediately used to transform chemically competent $E$. coli $\mathrm{DH} 5 \alpha$. To assess digest and ligation efficiencies, control reactions of uncut vector, cut vector and ligated cut vector without insert were used to transform competent cells in parallel.

\subsection{Overlap PCR for mutagenic fragment generation.}

For the generation of truncated gene fragments, two separate standard BioMix Red ${ }^{\mathrm{TM}}$ PCR reactions were carried out to produce left and right hand fragments. Using genomic DNA as a template, the $1 \mathrm{~kb}$ upstream flanking region and the $1 \mathrm{~kb}$ downstream flanking region of the target gene were amplified. These amplicons include the first three or last three codons from the respective $5^{\prime}$ or $3^{\prime}$ ends of the gene. A 25 bp homologous region of the $3^{\prime}$ end of the of the upstream/left-hand fragment were introduced by the primer to the $5^{\prime}$ 'end of the downstream/right-hand fragment. A restriction site was introduced by the primers into each fragment, upstream of the gene region for the left-hand fragment or downstream of the gene region for the right-hand fragment to allow for directional cloning of the final overlap product in to a knock out vector. Following column clean-up $150 \mathrm{ng}$ of each of these fragments was added to $50 \mu \mathrm{L}$ of $1 \mathrm{X}$ BioMix Red solution and thermocycled 4 times with a $50{ }^{\circ} \mathrm{C}$ annealing temperature to allow the homologous regions to hybridize and the spliced regions to self prime the extension of a complete mutagenic fragment. After the splicing step, outside primers were added and the reaction was thermocycled to amplify the spliced fragments. 


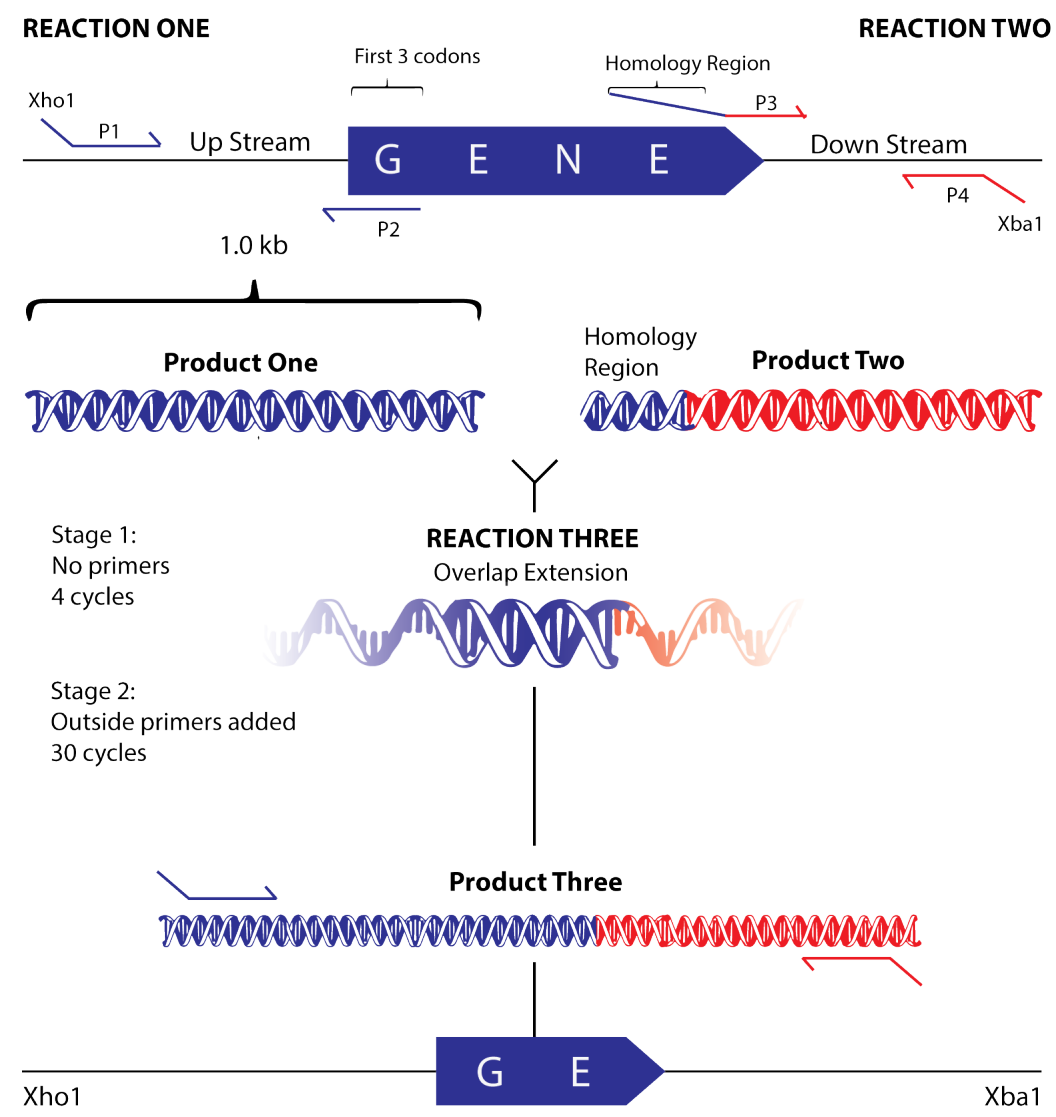

Figure 2.1: Splicing by overlap extension: This figure outlines the three PCR reactions and resulting products in the overlap extension protocol used to truncate target genes. Reactin one produces a $1 \mathrm{~kb}$ fragment that begins upstresm of the target gene (blue bar - GENE) and terminates at the third codon of the target gene. The $1 \mathrm{~kb}$ product of reaction two flanks the downstream region of the target gene capturing the last three codons and the forward primer used in this reaction incorporates a 25 bp sequence homologous to the $5^{6}$ end of this product that is homologous to the $3^{6}$ end of the product from reaction one. Reaction three splices the products from reaction one and two to generate the final product containing the truncated gene (blue bar - GE) which is then amplified. The primers that were used to amply the final product incorporate restriction sites (XhoI and XbaI in this figure) for cloning. 
Overlap PCR thermocycler protocol:

Table 2.9: Overlap PCR thermocycler protocol

\begin{tabular}{|c|c|c|}
\hline Temperature & Time & Cycles \\
\hline \multicolumn{3}{|c|}{ Initial denaturation } \\
\hline $95{ }^{\circ} \mathrm{C}$ & $5 \mathrm{~min}$ & \\
\hline \multicolumn{3}{|c|}{ Overlap splicing } \\
\hline $95^{\circ}$ & $30 \mathrm{~s}$ & \\
\hline $50^{\circ}$ & $30 \mathrm{~s}$ & 4 cycles \\
\hline $72{ }^{\circ} \mathrm{C}$ & $1 \mathrm{~min}$ & \\
\hline \multicolumn{3}{|c|}{ Outer primers added to reaction held at $95{ }^{\circ} \mathrm{C}$} \\
\hline $95^{\circ} \mathrm{C}$ & $30 \mathrm{~s}$ & \\
\hline $56{ }^{\circ} \mathrm{C}$ & $30 \mathrm{~s}$ & 10 cycles \\
\hline $72{ }^{\circ} \mathrm{C}$ & $1 \mathrm{~min}$ & \\
\hline $95^{\circ} \mathrm{C}$ & $30 \mathrm{~s}$ & \\
\hline $52{ }^{\circ} \mathrm{C}$ & $30 \mathrm{~s}$ & 20 cycles \\
\hline $72{ }^{\circ} \mathrm{C}$ & $1 \mathrm{~min}$ & \\
\hline \multicolumn{3}{|c|}{ Final extension } \\
\hline $72{ }^{\circ} \mathrm{C}$ & $10 \mathrm{~min}$ & \\
\hline $14^{\circ} \mathrm{C}$ & Hold & \\
\hline
\end{tabular}

\subsection{Preparation and transformation of competent cells}

2.7.1 Preparation and transformation of chemically competent $E$. coli

\section{Reagents:}

TFBI: Final 1X concentration - $30 \mathrm{mM} \mathrm{KOAc,} 50 \mathrm{mM} \mathrm{MnCl} 2,100 \mathrm{mM} \mathrm{KCl}, 10 \mathrm{mM}$ $\mathrm{CaCl}_{2}$. Salt solution made up to 10x. Stored at room temp. For use:

Dilute to $1 \mathrm{x}$ with ddH2O.

Add sterile glycerol up to $15 \% \mathrm{w} / \mathrm{v}$.

$\mathrm{pH}$ adjusted to 5.8 with $0.2 \mathrm{M}$ acetic acid.

Sterilized using a $0.22 \mu \mathrm{m}$ filter.

Cooled to $<4{ }^{\circ} \mathrm{C}$ in an ice bath.

TFBII: $10 \mathrm{mM}$ Na-MOPS pH 7.0, $10 \mathrm{mM} \mathrm{KCl,} 75 \mathrm{mM} \mathrm{CaCl2,} \mathrm{15 \%} \mathrm{w/v} \mathrm{glycerol.} \mathrm{This}$ solution was made up at $1 \mathrm{X}$ as needed and stored at room temperature, the solution was then sterilized using a $0.22 \mu \mathrm{m}$ filter and cooled in ice bath before use. 


\section{Preparation:}

Three mL LB was inoculated with a single colony of the desired strain of E. coli and incubated on an orbital shaker overnight at $37{ }^{\circ} \mathrm{C} / 200 \mathrm{rpm} .50 \mathrm{~mL}$ of $\mathrm{LB}$ in a $250 \mathrm{~mL}$ Erlenmeyer flask stopped with cotton wool and sealed with aluminium foil was autoclaved. $1 \mathrm{~mL}$ of overnight culture was used to inoculate the $250 \mathrm{~mL}$ flask with LB once cooled to room temperature. The flask was incubated on an orbital shaker at $37{ }^{\circ} \mathrm{C} / 200 \mathrm{rpm}$ for approximately 4 hours until the culture reached an OD600 of 0.3 to $0.5 .40 \mathrm{~mL}$ of this culture was transferred to a sterile $50 \mathrm{~mL}$ falcon tube and cooled on ice for $10 \mathrm{~min}$. The cells were then harvested in a refrigerated centrifuge set to $2500 \mathrm{rcf} / 4{ }^{\circ} \mathrm{C}$ for $10 \mathrm{~min}$. Supernatant was discarded and the cell pellet was resuspended in $2 \mathrm{~mL}$ ice cold TFBI by gentle pipetting. A further $38 \mathrm{~mL}$ of ice cold TFBI was then added to the tube. Cells were kept on ice for 2 hours and then harvested again in the same manner as before. The pellet was resuspended in $4 \mathrm{~mL}$ ice cold TFBII and $100 \mu \mathrm{L}$ aliquots were snap frozen in $1.5 \mathrm{~mL}$ tubes and stored immediately at $-80{ }^{\circ} \mathrm{C}$ until needed.

\section{Transformation:}

Cell aliquots were removed from $-80{ }^{\circ} \mathrm{C}$ storage and thawed on ice for 5 min. Ligation reaction or plasmid DNA was added to thawed cells and gently mixed. Aliquots were incubated on ice for $30 \mathrm{~min}$ followed by heat shock at $42{ }^{\circ} \mathrm{C}$ for 30 to 40 seconds. Aliquots were returned to ice for 5 mins and the cells were recovered by addition of $1 \mathrm{~mL}$ room temperature $\mathrm{LB}$ to the $1.5 \mathrm{~mL}$ tube. Tubes were then incubated at $37{ }^{\circ} \mathrm{C} / 200 \mathrm{rpm}$ for 40 to $60 \mathrm{~min}$. Transformed cells were harvested by centrifugation and the resulting pellet resuspended in $100 \mu \mathrm{L} \mathrm{LB}$ then plated on to appropriate antibiotic plates to select for plasmid containing cells.

\subsubsection{Preparation and transformation of electrocompetent $E$. coli}

\section{Preparation}

100x final desired volume of the desired strain was grown to log phase (OD600 0.3-0.4) under standard growth condition and then cooled on ice for 10 min. Culture was centrifuged at $2500 \mathrm{rcf}$ at $4{ }^{\circ} \mathrm{C}$ for $15 \mathrm{~min}$. The cell pellet was washed by resuspending in ice cold sterile $\mathrm{ddH}_{2} \mathrm{O}$ and centrifuged again as before. The wash step was repeated once more and the pellet was finally resuspended in a volume of ice cold MQ that is $1 / 100^{\text {th }}$ that of the starting culture's original volume.

\section{Transformation}

Each transformation reaction used $60 \mu \mathrm{L}$ of the prepared cell suspension. To this, up to 25 $\mu \mathrm{L}$ of plasmid or linear DNA preparation in MQ was added. The reaction mixture was transferred to an ice cold $2 \mathrm{~mm}$ electroporation cuvette and pulsed in a GenePluser Xcell ${ }^{\mathrm{TM}}$

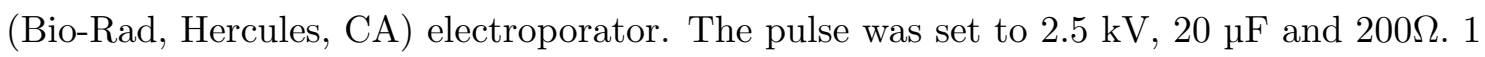
$\mathrm{mL}$ of ice cold LB was added to the cuvette immediately after the pulse was administered then the reaction was allow to return to room temperature before incubation at $37^{\circ} \mathrm{C}$ for 40 
to $60 \mathrm{~min}$. The cells were harvested by centrifugation and plated on to selective media to isolate transformants.

\subsubsection{Preparation and transformation of electrocompetent Pseudomonas.}

This method was used for the preparation of electrocompetent Pseudomonas syringae pv. actinidiae and P. syringae 1448a and is based on a protocol described in [126] A $50 \mathrm{~mL}$ culture of the desired strain was grown until stationary phase under the appropriate conditions by inoculation of $50 \mathrm{~mL} \mathrm{LB}$ with $1 \mathrm{~mL}$ of overnight culture and incubated for 16 to 24 hours. For each competent cell aliquot to be prepared $6 \mathrm{~mL}$ of culture was aliquoted into four $1.5 \mathrm{~mL}$ sterile microcentrifuge tubes. Cells were then pelleted by centrifugation (17,000 rcf, $30 \mathrm{~s}$, at room temperature) and each pellet washed twice under sterile conditions in $1 \mathrm{~mL}$ of sterile $300 \mathrm{mM}$ sucrose. After the final wash step the four cell pellets were resuspended in a final volume of $100 \mu \mathrm{L}$ sterile $300 \mathrm{mM}$ sucrose. For transformation with an non-replicating integrating KO plasmid (pDM4 or pEX18 Gm) $400 \mathrm{ng}-1 \mathrm{\mu g}$ of plasmid DNA in midi-prep elution buffer was added to the aliquot as a volume of 5-10 $\mathrm{LL}$ depending on DNA concentration available. This was then transferred to a $2 \mathrm{~mm}$ gap electroporation cuvette and electroporated with pre-set pulse setting of $2.5 \mathrm{kV}, 25 \mu \mathrm{F}, 200$ Sshunt resistor. Immediately after pulsing, $1 \mathrm{~mL}$ of LB was added to the cuvette and then the entire contents were transferred to a $15 \mathrm{~mL}$ tube for recovery. Recovery time was 1 hour at appropriate growth conditions. After recovery, cells were harvested via centrifugation and resuspended in $100 \mu \mathrm{L}$ of media for plating on to antibiotic containing selective LB agar plates and incubated until colonies formed. All preparation steps were performed at room temperature. For each batch of aliquots prepared, a control aliquot was electroporated with sterile MQ substituted in place of the DNA prep and plated alongside the experimental aliquots.

\subsubsection{Transformation by bacterial conjugation as a means of plasmid transfer into Pseudomonas species.}

A culture of host/donor strain E. coli DH5 $\alpha$ pir for triparental conjugation, or S17-1 for biparental conjugation, was prepared by transformation with the desired plasmid to conjugate using the chemical competency method. A colony verified to contain the desired plasmid was then used to inoculate $5 \mathrm{~mL}$ of LB plus the appropriate antibiotic and incubated at $37{ }^{\circ} \mathrm{C} / 200 \mathrm{rpm}$ overnight until stationary phased was reached. The E. coli helper strain ANS98 for triparental conjugation was grown in the same conditions without antibiotics. The recipient strain to be transformed was prepared by inoculating from glycerol stock $5 \mathrm{~mL}$ of $\mathrm{LB}$ and incubated overnight at $30{ }^{\circ} \mathrm{C} / 200 \mathrm{rpm}$. A day culture of each strain was set up by dilution of the overnight preparations 1:10 in LB, with antibiotic supplementation for the host strain, and incubated with shaking until an OD600 of $\geq 1.0$ was reached. Host strains were washed twice in fresh LB to remove antibiotics. The recipient strain was heat shocked by incubation at $50{ }^{\circ} \mathrm{C}$ for $4 \mathrm{~min}$ and then placed on ice until required.

To a $1.5 \mathrm{~mL}$ microcentrifuge tube $0.75 \mathrm{~mL}$ of host and recipient strains were added and 
mixed by inversion, and $0.25 \mathrm{~mL}$ of the helper strain was substituted to the mixture if required for triparental conjugation. Cells were pelleted by brief centrifugation, resuspended in $50 \mu \mathrm{L}$ of $\mathrm{LB}$ and pipetted on to LB agar plates. Plates were allowed to dry for $20 \mathrm{~min}$ in a biological safety hood then incubated at $37{ }^{\circ} \mathrm{C}$ overnight. The next day the patch of cells were transferred by sterile loop to Psa-V selective media agar plates with the appropriate antibiotic to select for recipient cells maintaining a plasmid.

\subsection{Screening to identify recombinant $E$. coli clones}

\subsubsection{Colony PCR}

Colony PCR used bacterial cells harvested on the fine point of a pipette tip or sterile toothpick from an agar plate and delivered into the PCR components in place of the gDNA template. The standard BioMix Red PCR conditions were used except the initial $95{ }^{\circ} \mathrm{C} 5$ min melting phase is increased to $98{ }^{\circ} \mathrm{C} 5$ min to encourage the lysis of cells and expose the genetic material to the reaction conditions. Colonies to be screened were first selected from the transformation plate and catalogued on to a fresh plate containing appropriate antibiotic supplemented LB agar. Each transferred colony was assigned a number inscribed on to the outside of the plate with a permanent marker. The catalogue plate was then incubated overnight, or until colonies form, at strain specific growth conditions. The catalogued colonies were used as a template in colony PCR to control for amplification from ligation reaction products spread on to the initial transformation plate. If possible a forward primer complementary to the plasmid sequence was used in conjunction with a reverse primer complementary to the expected insert sequence to control for non-specific amplification from the genome. When this was not available only insert specific primers were used. Where possible an extracted and purified DNA positive control was used. A colony containing the plasmid without an insert was analysed as a negative control.

\subsubsection{Confirmation by restriction digest}

Once a colony maintained on the catalogue plate was identified as containing the desired plasmid::insert construct the catalogue plate was used as a source of inoculum to prepare a $3 \mathrm{~mL}$ overnight culture at strain and plasmid appropriate conditions. The plasmid construct was extracted and purified from the culture using a mini-prep spin column. This plasmid prep was then subject to restriction digest with enzymes that cut out the insert from the plasmid leave linear DNA fragments. The same combination of enzymes were used to linearise empty circular plasmid for comparison. For diagnosis the digested plasmid preps and undigested plasmid preps were analysed by electrophoresis. A positive control of unligated insert was analysed. A banding pattern showing linearised plasmid and excised insert was expected in the diagnostic lane. 


\subsection{In-frame deletion of genes from the chromosomes of Psa-V and Ps 1448a.}

To generate the truncated gene mutagenic fragments to replace chromosomal genes a set of primers were designed to amplify two $1.0 \mathrm{~kb}$ products that contained a $25 \mathrm{bp}$ region of homology. The $1.0 \mathrm{~kb}$ were spliced together using the overlap PCR protocol resulting in a $\sim 2.0 \mathrm{~kb}$ product that has $\sim 1.0 \mathrm{~kb}$ of sequence identical to the $1.0 \mathrm{~kb}$ region upstream of the gene and $\sim 1.0 \mathrm{~kb}$ of sequence identical to the downstream region. Between the two gene flanking homologous regions of the product was a truncated version of the gene consisting of only the first 3 codons and the last 3 codons. This design maintains the original reading frame of the gene when the fragment is integrated into the chromosome. Fragments were restriction digested and then ligated into the pEX18Gm vector that had been digested with the restriction enzymes required to produce sticky ends compatible with the insert.

Ligation reactions were carried out following the protocol outlined in section 2.5.1. Ligation products were used to transform chemically competent $E$. coli DH5 $\alpha-\lambda$ pir and recombinant clones were identified and isolated as previously described in section 2.8. A successfully transformed colony was used as inoculum for a $50 \mathrm{~mL}$ culture that was subject to midi-prep plasmid extraction and purification. This plasmid preparation was used in the electroporation protocol to transform the desired strain of Pseudomonas. Cells that had achieved primary integration were selected for by plating on to solid media containing $20 \mathrm{\mu g}$ gentamicin. After 48 hours of incubation colonies that were resistant the antibiotic were screened for primary integration of the vector by colony PCR. Colonies returning positive for primary integration were forced to undergo a second recombination event by culturing overnight in LB containing $5 \% \mathrm{w} / \mathrm{v}$ sucrose, harvested by brief centrifugation and plated on to solid LB containing $10 \% \mathrm{w} / \mathrm{v}$ sucrose. Single colonies that formed on sucrose media were catalogued on to LB plates with and without gentamicin. Reversion to antibiotic sensitivity via the loss of the resistance gene contained in the plasmid DNA was assumed to indicate a second recombination event. Colony PCR was used to screen for the integration of the mutagenic fragment into the chromosome and for the lack of plasmid DNA.

\subsection{Deletion of genes from $E$. coli strains}

Disruption of chromosomal genes in E. coli was achieved by adapting the protocol as published by Datsenko and Wanner, (2000) [127]. The target strain was transformed with the pKD46 plasmid $\left(\mathrm{Amp}^{\mathrm{R}}\right)$, which carries the phage $\lambda$ Red recombinase under control of an arbinose inducible promoter. A log phase culture of the target strain carrying pKD46 was induced by supplementing the growth media with IPTG to a final concentration of 2 $\mathrm{mM}$ and incubated for a further 4 hours at $37^{\circ} \mathrm{C}$. This culture was then prepared for electroporation, section 2.7.2. A linear knock out DNA construct with target gene homology and a kanamycin resistance cassette is electroporated into the cells. The knock out construct was prepared by amplifying the kanamycin resistance cassette from the template plasmid pKD4 with primers that incorporate 35 bp of homology to the flanking regions of the target gene into the PCR product. The PCR product was treated with DpnI 
prior to electroporation, degrading the original template plasmid. After electroporation the cells were recovered in rich media supplemented with IPTG for 4 hours to allow recombination to occur between the knockout construct and the bacterial chromosome. Recombination was selected for by plating the cells on LB agar with kanamycin. A single recombined colony from the kanamycin plate was used to inoculate $3 \mathrm{~mL}$ of unsupplemented LB and incubated overnight at $42{ }^{\circ}$ with $200 \mathrm{rpm}$ orbital shaking. A high incubation temperature served to cure the pKD46 plasmid. Individual colonies were counter selected on ampicillin and kanamycin plates, colonies that had lost ampicillin resistance were screened for gene knock out by PCR.

\subsection{SDS-polyacrylamide gel electrophoresis}

SDS-PAGE was performed using $12 \%$ SDS-polyacrylamide gels prepared according to the method of Laemmli [128]. Gels were cast and run using the Biorad Protean II TM system, according to the manufacturers directions. Preparation of samples, as well as gel staining and destaining, was carried out as described by Sambrook and Russell [129].

\subsubsection{Molecular weight markers}

The molecular weight marker for the estimation of protein size employed in this study was the NEB broad range molecular weight marker.

\subsection{Artificially induced spontaneous streptomycin resistance mutation}

An individual colony of Psa-V growing on LB agar was picked off with a sterile loop and used to inoculate $3 \mathrm{ml}$ of LB which was then incubated overnight. Eight $15 \mathrm{ml}$ sterile tubes were set up and seven of the tubes contained $3 \mathrm{ml}$ of LB in and labeled as a series from two to eight. The first tube contains $6 \mathrm{ml}$ of a $30 \mathrm{mM}$ streptomycin sulphate solution. A dilution series was then created in these tube. A volume of $3 \mathrm{ml}$ was aspirated from tube one and delivered to tube two. Tube two was mixed and $3 \mathrm{ml}$ of this mixture was transferred into tube three. This process was repeated for the entire series of all eight tubes and the most dilute solution of the series in tube eight was $234 \mu \mathrm{M}$, this concentration of streptomycin is sub-lethal to PsaV. All tubes in the series were inoculated with $20 \mu \mathrm{L}$ of the overnight Psa-V and then incubated for 48 hours. After this time the culture with the highest concentration of streptomycin that had visible bacterial growth was used to re-inoculate the remaining tubes of higher concentration. This was repeated until the tube with the top concentration maintained a viable culture. The process was also carried out using a dilution series in solid media using narrower concentration increments although within the same range as the liquid dilution series. 


\subsection{Antimicrobial testing}

Antimicrobial testing was carried out in 96 well plates. Growth inhibition was assessed the difference in OD600 readings of treated and untreated cultures. The cultures were exposed to six replicate serial dilutions of the antimicrobial in question in rich media and after incubation at optimal conditions for at least 24 hours, which allows untreated cultures to reach stationary phase, the OD600 readings of each culture in the series was recorded. The relative growth of the cultures in each well of the serial dilution plate was determined by the comparison of their OD600 reading to that of cultures in untreated control wells. Control wells contained only the same volume of inoculated rich media.

\subsubsection{Details of serial dilution plate set up, challenge and recording.}

1. Under sterile conditions a new 96 well plate was removed from the sterile packaging. Columns 1-11 were filled with $100 \mu \mathrm{L} \mathrm{LB}$ (or other appropriate growth medium). Column 12 empty was left empty .

2. Column 3 filled with $100 \mu \mathrm{L}$ - ( $\mu \mathrm{L}$ of compound to be tested) LB. (e.g. If $20 \mu \mathrm{L}$ of test compound was required to reach the desired top concentration then $80 \mu \mathrm{L}$ of LB was added (Fig. 2.2)

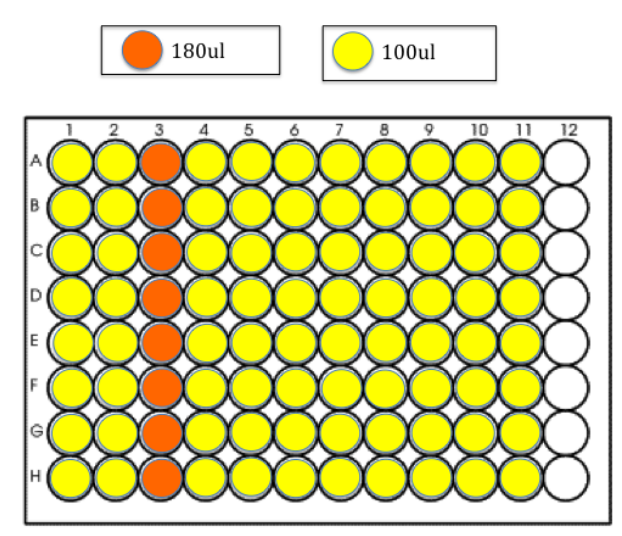

Figure 2.2: Assay plate set up step 2

3. Test compounds were added, in duplicate or triplicate, into rows B-G of column 3 to bring the volume of in all test rows up to $200 \mu \mathrm{L}$ (Fig. 2.3). 


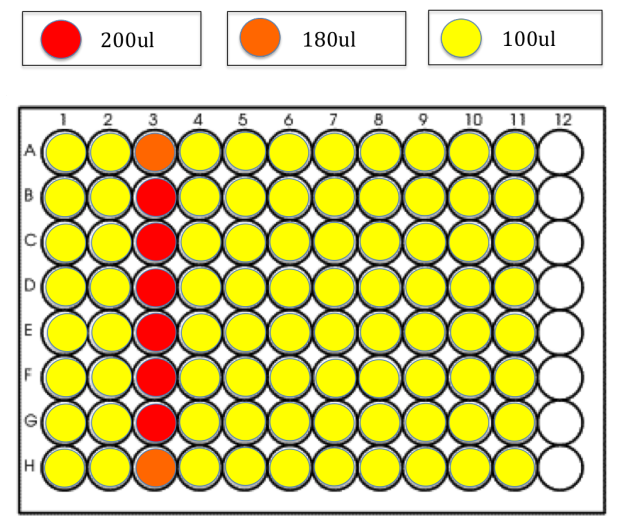

Figure 2.3: Assay plate set up step 3

4. Next, LB was added to bring outside test column wells up to $200 \mu \mathrm{L}$. A serial (two-fold) dilution was performed across the columns of the plate, $100 \mu \mathrm{L}$ from all wells in column 3 and delivered to column 4 . This was repeated from column 4 to column 5 and continued along until $100 \mu \mathrm{L}$ was finally delivered into column 12. Each transfer was gently pipetted up and down to mix. All wells contained $100 \mu \mathrm{L}$ at the end of this process. (Fig. 2.4).

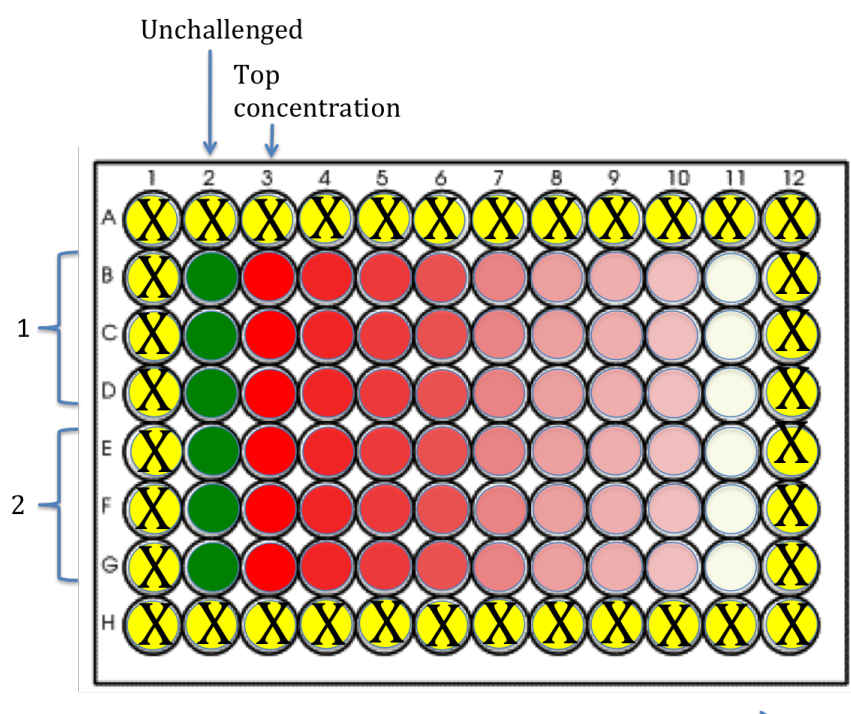

Concentration Gradient

Figure 2.4: Test plate immediately prior to culture challenge.

\subsubsection{Culture preparation}

The following procedure was used to prepare the challenge culture.

1. $3 \mathrm{~mL}$ fresh $\mathrm{LB}$ was added into a $50 \mathrm{~mL}$ falcon tube.

2. This was inoculated with a verified Psa-V strain. Using a sterile pipette tip one colony was picked from an agar plate and ejected into the falcon tube with $3 \mathrm{~mL}$ LB.

3. Another $50 \mathrm{~mL}$ falcon with $3 \mathrm{~mL} \mathrm{LB}$ and a clean pipette tip was used as media control. 
4. Incubated over night $\left(16 \mathrm{hrs} / 30^{\circ} \mathrm{C} / 200 \mathrm{rpm}\right)$.

5. At post 16 hours incubation time, the culture falcon was topped up with LB to the 50 mL mark. Checked OD600. OD600 should be below 0.1. (around 0.05-0.07) If not, it was diluted with LB until it was below 0.1.

6. The $50 \mathrm{~mL}$ sub OD600 $=0.1$ culture was returned to the incubator and recover until the $\mathrm{OD} 600=0.1$.

\subsubsection{Culture challenge and plate reading}

The following procedure was used to challenge the prepared test plate.

1. Once culture is within $10 \%$ of $\mathrm{OD} 600=0.1$, pour in to a suitable vessel to use with $8 \mathrm{x}$ multichannel pipette.

2. Deliver $100 \mu \mathrm{L}$ of this culture to all test wells of the prepared 96-well serial dilution plate.

3. Wells around the perimeter are not used to record measurements, to avoid the edge effect due to the evaporation that can occur in these wells.

4. Label plate with compound/s, date, plate number. Read OD600 in plate reader. This reading is $\mathrm{T}=0$ and was used as a background reading.

5. Incubate plate for 16 to 24 hours at $30{ }^{\circ} \mathrm{C} / 200 \mathrm{rpm}$.

6. Reread plate in plate reader. This is $\mathrm{T}=$ challenge time.

7. Relative growth for an individual well is calculated as a fraction of the average readings of the unchallenged wells.

E.g. $(O D 600$ at $T=$ challenge time $-O D 600$ at $T=0) /$

$O D 600$ at $T=$ challenge time $-O D 600$ at $T=0$. For each element in the dilution series, the average relative growth of the six replicates wells per plate was plotted over the respective concentration of that element then a dynamic curve is fitted to this data. The four-parameter dose-response curve function of Sigma plot was used to report the $\mathrm{IC}_{50}$ value and error. Briefly, rather than the standard (-1) slope, the fitted sigmoidal curve is allowed a non-standard hill slope which is instead fitted to the data. The $\mathrm{IC}_{50}$ value is calculated at half way between the top and bottom (y-axis) plateaus of this variable slope curve.

\subsection{Competitive growth fitness assay of antibiotic resistant strains}

Assays to measure the relative fitness between co-inoculated antibiotic resistant and wild type strains growing competitively in rich media.

\subsubsection{Rich media based co-culture growth competition assay}

Over night stationary phase cultures of each strain to be tested were passaged again in rich media (LB) to synchronise. A $50 \mathrm{~mL}$ tube containing $5 \mathrm{~mL}$ of $\mathrm{LB}$ was co-inoculated with 5 
$\mu \mathrm{L}$ of each of the synchronised cultures. Separate tubes inoculated with $5 \mu \mathrm{L}$ of the individual strains were set up in parallel as controls. These tubes were incubated for 24 hours (at $28^{\circ} \mathrm{C}$ for PsaV) to allow cultures to reach stationary phase. To calculate the relative densities of each strain in a sample $5 \mu \mathrm{L}$ was aspirated from each sample tube and introduced to a fresh $50 \mathrm{~mL}$ tube containing $5 \mathrm{~mL}$ of LB to give a 1:1000 dilution. A 100 $\mu \mathrm{L}$ sample of the diluted culture was taken and diluted twice more to give $1 \mathrm{~mL}$ samples with final dilutions of 1:10,000 and 1:100,00. A $100 \mu \mathrm{L}$ aliquot of each of these two final dilutions were plated on to LB agar with and without the antibiotic of interest. These plates were incubated until colonies formed then colony counts were taken from the plates that gave the most accurate reading. This was usually from the 1:100,000 diluted sample. A normalised $\mathrm{CFU}$ value for the resistant and non-resistant strains was calculated for each

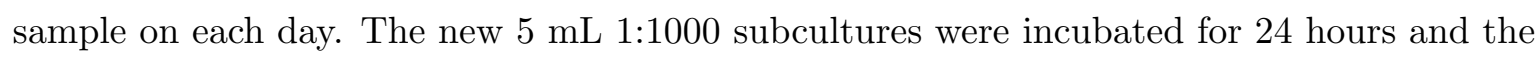
process was repeated every day for 12 days.

\subsubsection{Kiwifruit seedling based co-culture growth competition assay}

An assay to measure the relative fitness between co-inoculated antibiotic resistant and wild type strains of Psa-V growing competitively in Hort 16A kiwifruit plant seedlings. The clonal, cell culture propagated seeding used in the following method were supplied by Plant and Food Research. The processing of samples and plant cultivation during the course of the experiments using the following method was carried out in the Beever plant pathogen containment facility at Landcare Research, Tāmaki, Auckland.

\section{Preparation}

\section{Day 1}

Preparation of plants:

Sixteen seedlings for each treatment were placed in separate tubs with standing water and cultivated in a PC2 green house. The plants were labeled appropriately by treatment. The treatments included: inoculation with wild type PsaV, inoculation with streptomycin resistant PsaV and co-inoculation with an equal mixture of both strains.

Preparation of inoculum strains and sample tubes:

Each strain was streaked out onto KB agar and incubated overnight at $28^{\circ} \mathrm{C}$. Strains had previously been revived from glycerol storage between 2 days and 3 weeks prior. Sterile 1.5 $\mathrm{mL}$ microcentrifuge tubes were set out and labeled appropriately for each tissue sample, 3-4 sterile stainless steel beads and $200 \mu \mathrm{L} 10 \mathrm{mM} \mathrm{MgSO}_{4}$ was added to each tube. The weights of each tube loaded with beads and $\mathrm{MgSO}_{4}$ was recorded.

Day 2

Inoculum solution:

The final inoculum solution was made by resuspending a small loopful of fresh cell in $1 \mathrm{~mL}$ of $10 \mathrm{mM} \mathrm{MgSO}_{4}$ and determining the OD600 of the suspension, then by dilution in $10 \mathrm{mM}$ 
$\mathrm{MgSO}_{4}$, a $1 \mathrm{~mL}$ solution of each strain was prepared to an OD600 of 0.2 using the resuspended cells. For the co-inoculation treatment, $500 \mu \mathrm{L}$ of each OD600 $=0.2$ solution was mixed together.

Seedling inoculation:

Seedlings were inoculated in the main stem 3-5 mm below the first fully formed leaf by dropping $2 \mu \mathrm{L}$ of the inoculum solution onto the stem and then perforating the stem with a sterile needle.

\section{Growth assay}

In planta bacterial growth was measured at three time points over seven days. These time points were at post inoculation days 0,3 and 7 . On the day 0 time point four inoculated seedling per treatment were sampled, for the remaining time points (days 3 and 7) six plants per treatment were sampled. Samples of plant tissue were taken and processed as follows to measure bacterial growth in the plants.

The tool and materials required for tissue sampling were set out in a class 2 biological safety cabinet. This included: scissors, tweezers and cork borer were sterilised with $100 \%$ EtOH. A solution of $70 \%$ EtOH for surface sterilisation of plant tissue samples. Labeled microcentrifuge tubes were pre-filled with $200 \mu \mathrm{L}$ of $10 \mathrm{mM} \mathrm{MgSO}_{4}$ and sterile beads. A 96 well dilution plate, pipettes, tips and labeled KB agar plates ( \pm streptomycin where appropriate) supplemented with nitrofurantoin at $50 \mu \mathrm{g}$ per $\mathrm{mL}$.

For each plant, one stem section $(<10 \mathrm{~mm}$ long) from the site of inoculation and one leaf punch, including the midrib, from the base of the leaf directly above the inoculation site was harvested.

Tissue samples were surface sterilised for $10 \mathrm{~s}$ in $70 \% \mathrm{EtOH}$. Stem sections and leaf discs were dried lightly on a sterile Kimwipe to remove excess EtOH and then placed in the appropriately labeled microcentrifuge tube. All tools were sterilised in $100 \% \mathrm{EtOH}$ and then heated in a sterilising furnace between each sample.

Stem sample tubes were weighed and all samples were homogenised by bead mill. The 96-well dilution plate was then prepared, first $90 \mu \mathrm{L}$ of $10 \mathrm{mM} \mathrm{MgSO}_{4}$ was added to all wells of the $2 \mathrm{nd}, 3 \mathrm{rd}, \ldots, 12$ th columns.

The first column was loaded by adding $100 \mu \mathrm{L}$ of a homogenated sample into the first well of a row, using a new row for each replicate. The homogenates were serially diluted at a factor of 1:10 by transferring $10 \mu \mathrm{L}$ from the wells of column one into the respective wells of the next column, mixing at each step by pipetting up and down slowly, and repeating this for each column. Tips were discarded between each transfer.

A $10 \mu \mathrm{L}$ sample of each dilution was dropped onto a labeled KB plate and incubated at $28^{\circ} \mathrm{C}$ for two days. After two days colonies became be visible on the plate. A spot with between 10-100 colonies was observed under magnification and the number of individual colonies and the dilution of the spot was recorded, the initial in planta bacterial density per $\mathrm{cm}^{2}$ (or $\mathrm{mg}$ ) of leaf area was then calculated. 


\section{Chapter 3}

\section{Experimental Results - Antimicrobial Agents}

\subsection{Introduction}

This chapter presents the results of a detailed analysis of chemical antimicrobial control agents that might have applications in combating the Psa-V epidemic. This work is divided into several sections, starting with the development and implementation of a quantitative in-vitro screen to evaluate candidate antimicrobial compounds against Psa-V. Following on from this, to expand the range of control options a complex mixture of a novel antimicrobial agent combined with a synergistic "helper" compound was analysed for activity against Psa-V. In this combination, the "helper" compound greatly increased activity, most likely by blocking efflux pumps in Psa-V. Next, the possibility of Psa-V developing resistance to the registered antibiotics already in use as chemical controls in the field was tested.

Spontaneous streptomycin resistance was experimentally induced in Psa-V, and the mechanism and fitness impact associated with this resistance phenotype were investigated.

Chemical spraying of orchards is a management practice that has been used by kiwifruit growers to control pests and diseases since at least 1950 [130]. In the past this control was used to maintain plant health in areas of high production intensity and increase yield to meet export supply and quality demands [131]. Copper-based sprays have been successfully used as a protective measure against bacterial diseases in kiwifruit, e.g. the pre-2008 incursions of Psa in Italy. Also, streptomycin (either alone or in combination with oxytetracycline) has been successfully employed as a spray or via direct injection into vines to control P. syringae on and in kiwifruit in Japan and Korea [132, 133]. Streptomycin is a particularly attractive candidate for use in New Zealand kiwifruit orchards, as it is affordable, highly effective against sensitive phytopathogen strains and it is already widely employed to control bacterial pathogens in other economically important crops (e.g. fire blight - Erwinia amylovora in apple orchards). However, the practice of using streptomycin to protect apples from fire blight has led to the emergence of streptomycin resistant strains of E. amylovora in these orchards [134]. Strains of P. syringae that are streptomycin resistant have also been isolated from apple orchards in New Zealand where this antibiotic was used [135]. In other countries where streptomycin is used (e.g. in the USA for $E$. amylovora control) it is sometimes combined with oxytetracycline to preserve the efficacy of streptomycin by reducing the occurrence of resistance. Although dual antibiotic use was considered unfavourable by these growers because of increased cost (and thus it was poorly adopted), the risk of promoting antibiotic resistance is greatly mitigated if two antibiotics 
with different targets/modes of action are used in conjunction [136]. Because of the previous positive results seen with the use of antibiotics on kiwifruit and other crops there was eagerness to have these agents available to manage Psa-V in New Zealand [137, 138]. KeyStrepto ${ }^{\circledR}$ was approved for use in August 2011, but strategies to reduce the problems of resistance, residue build up and phytotoxicity must be considered. Thus, Seeka Kiwifruit Ltd, the kiwifruit cooperative co-funding this work, had a strong interest in not only testing the efficacy of streptomycin against Psa- $\mathrm{V}$, but also identifying other antimicrobial agents that they might be able to apply for approval to spray in conjunction with streptomycin (Michael Franks, CEO Seeka Kiwifruit Ltd, personal communication). It is the opinion of the New Zealand Plant Protection Society (NZPPS) that streptomycin use should be ceased at the first sign of a lack of efficacy until the presence or absence of resistant strains of the pathogen can be confirmed by a microbiological laboratory. It is also recommended by the NZPPS that streptomycin use is alternated with other antibacterial compounds where possible as part of a resistance management strategy [134].

Kasugamycin was flagged as a logical candidate for use in conjunction with KeyStrepto. This antibiotic is affordable, not used clinically in humans and is inhibitory toward species of Pseudomonas, Erwinia, Xanthomonas, as well as several fungal plant pathogens. Kasugamycin has previously been used in a horticultural setting with success in Japan where it was employed for the management of rice blast caused by Pyricularia oryzae [139]. Kasugamycin was recommended by researchers as a second antibiotic control agent for use in New Zealand kiwifruit orchards and approved with controls in October of 2013 by the Environmental Protection Agency. The official submissions and approval documentation are publicly available at www.epa.govt.nz [138].

On top of the outlay cost to the industry of registration, purchasing and application, a commercial concern specifically regarding the use of antibiotics is that it reduces the export value of the crop, for example the European Union restricts the import of fruit containing any antibiotic residues. Thus, Seeka Kiwifruit Ltd also expressed a strong interest in testing alternative antimicrobial compounds or solutions that are not recognised as traditional antibiotics. Because they have a large number of concerned stakeholders or other interested parties sending them alternative remedies, some of which appear scientifically valid and others less so (Michael Franks, CEO Seeka Kiwifruit Ltd, personal communication), this project sought to test a number of the most plausible candidates to establish anti-Psa-V activity and thereby reduce the number of samples that Seeka-employed scientists would go on to test in simulated field trials.

For these purposes, we initially sought to optimise a simple yet accurate assay to produce Psa-V $\mathrm{IC}_{50}$ data for compounds of interest.

\section{$3.2 \quad \mathrm{IC}_{50}$ screening of antimicrobials}

\subsubsection{Assay design}

The assay developed in this study sought to provide a quantitative measure of the concentration (or, for complex mixtures of unknown concentrations, the dilution factor) of a 
test compound needed to effectively inhibit Psa-V growth. Data was produced to calculate of the concentration of an antimicrobial where the total growth inhibition response is reduced by half $\left(\mathrm{IC}_{50}\right)$. This allows a comparison of effectiveness between treatments. Following several pilot efforts (not shown), a versatile protocol was developed according to which a dilution series of one or more test compounds was established in the inner 60 wells of a 96-well plate (the outer wells being filled with media only to minimise desiccation of the inner 60 wells). A range of concentrations wide enough to yield a full dose response was ideally represented for each compound, requiring initial optimisation of the top concentrations and dilution factors used. The plates were then inoculated with replicate cultures of Psa- $\mathrm{V}$ of a predetermined density and incubated at optimal conditions until the negative treatment controls reached a stationary phase (Fig. 3.1). Full details of this assay are discussed in section 2.13.

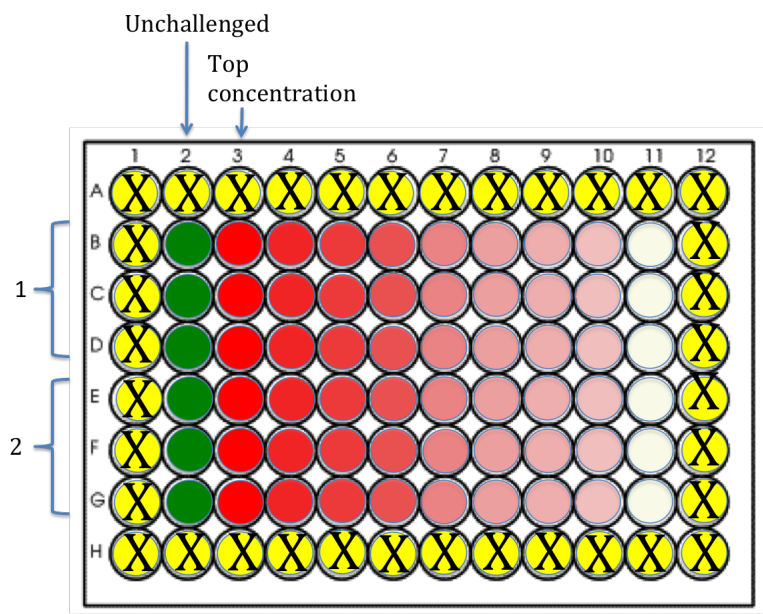

Concentration Gradient

Figure 3.1: Schematic of test plate set up: Depicted is a microtiter plate assay based on the optical density (OD at $600 \mathrm{~nm}$ ) of replicate Psa-V cultures in liquid media, which was challenged by a serial dilution of each test compound (starting from 10 to $0.1 \mu \mathrm{L}$ of compound solution added to the top concentration wells, as described in the main text). The gradient of red represents the concentration of the test compound, with green representing the unchallenged (no test compound added) negative control wells.

\subsubsection{Assay preparation and optimisation}

Preliminary tests were conducted with each test compound to establish the concentration range needed to achieve a dose dependent response and to also return enough data points to fit a non-linear regression curve allowing accurate analysis. For solid compounds (e.g. metal salts or analytical grade antibiotics), unless stated, a standard stock concentration of 50 $\mathrm{mg} / \mathrm{mL}$ was prepared in $\mathrm{ddH}_{2} \mathrm{O}$ where possible, otherwise in DMSO. Vehicle controls for these solvents showed no detectable effect on growth rate or final cell density and typically made up $<1 \%$ of the growth media in the top concentration wells. The controls were run as direct comparison of cultures \pm the greatest volume of vehicle used overall in these experiments, for $\mathrm{ddH}_{2} \mathrm{O}$ the greatest volume amounted to $5 \%$ total volume and for DMSO $0.5 \%$ total volume. In only one instance was the volume of test solution large enough to cause an effect, due to growth media dilution, and this was controlled by preparing the test solution in growth media rather than $\mathrm{ddH}_{2} \mathrm{O}$. Pre-formulated solutions were administered in 


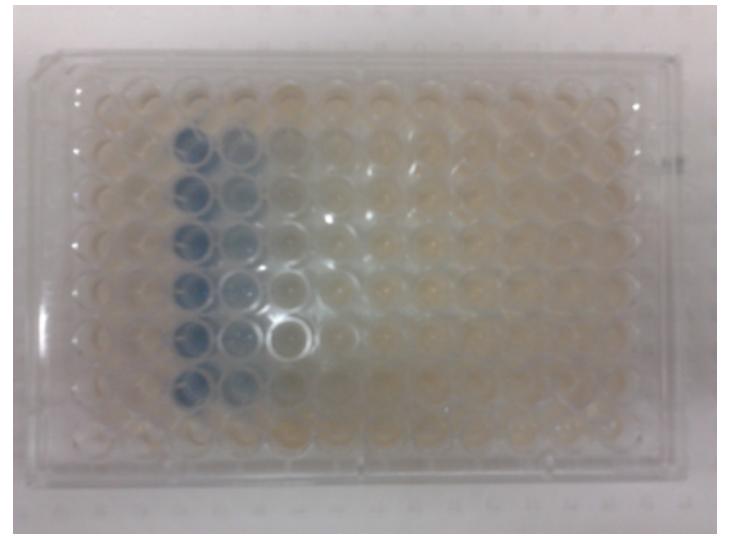

Figure 3.2: $\mathbf{C u S O}_{4}$ tests plate:The high concentrations of copper sulphate needed for inhibition is apparent in the leftmost test wells (no growth inhibition was observed in wells where blue colour was not readily discernible by eye).

unaltered form. Once a suitable concentration range for each test compound had been established empirically, a serial dilution was prepared under aseptic conditions. Most test compounds were evaluated across a two-fold dilution series. The most concentrated dilutions used were between $0.1 \mu \mathrm{L}$ and $10 \mu \mathrm{L}$ of test compound in a total well volume of $200 \mu \mathrm{L}$. The top concentration was designed to give complete inhibition of cell growth $\left(\mathrm{IC}_{100}\right)$ while allowing undetectable inhibition at the lowest concentration $\left(\mathrm{IC}_{0}\right)$. For several of the commercial compounds a two-fold dilution increment did not produce adequate data points to produce a smooth dose curve. In these cases a 1.25 fold increment series was set up. Once a concentration range had been optimized for all compounds, replicate cultures were challenged with at least six identical serial dilutions for 24 hours under standard growth conditions.

In several cases, initial testing was confounded by compounds that became very opaque upon dilution in growth media or by compounds that were strongly pigmented. However, no compounds with these properties were found to possess substantial anti-Psa-V activity, so collection of accurate $\mathrm{IC}_{50}$ data was not pursued. In these cases, qualitative zone of inhibition tests were used to confirm lack of efficacy. As discussed, the limitation of certain low-activity compounds (that required high concentrations for a substantial antimicrobial effect) was the effect of diluting the culture media, or requiring concentrations inducing high levels of osmotic stress. Both of these issues were seen with the copper sulphate solution (Fig. 3.2). Unless data was specifically requested by Seeka Kiwifruit Ltd for such compounds, collection of accurate $\mathrm{IC}_{50}$ data was again not pursued.

\subsubsection{Candidate compounds}

Candidates for antimicrobial testing against Psa-V were selected primarily on the basis of compounds or formulas of unknown efficacy that were available to growers, and compounds or formulas of unknown efficacy that Seeka Kiwifruit Ltd. were interested in potentially registering for use. In total, more than 30 commercial or experimental products were sent to the Ackerley lab by Seeka Kiwifruit Ltd for testing. All compounds were tested but only those that performed in the assay are included below in Table 3.1. This collection was supplemented with antibiotics purchased from Sigma-Aldrich Ltd, or from existing lab 
stocks (this included several clinically-used antibiotics that would not be appropriate for field testing but were relevant either as positive controls or as possible selection agents of relevance to the later stages of this research). Finally, a solution of silver nanoparticles produced by Professor Jim Johnston's group in the School of Chemical and Physical Sciences, VUW, was also included, as interest was expressed from Seeka in possible spray or injection tests of these nanoparticles as anti-Psa-V agents. For subsequent field or spray tests to be conducted by Seeka-employed scientists, antimicrobial candidates were narrowed down to those that showed a moderate to high level of activity against Psa-V, guided by the rationale that only highly effective candidates would be likely to be useful in the field.

Candidate compounds requiring high concentrations to achieve antimicrobial activity would not be reliable nor economically attractive to kiwifruit growers, and may lead to excess run-off causing environmental issues and possible phytotoxicity as seen previously with copper [140]. 
Table 3.1: Candidate compounds showing efficacy in this study

Clinical antibiotics

Gentamicin

Chloramphenicol

Oxytetracycline

Field use antibiotics

Streptomycin

Kasugamycin

\section{Metal compounds}

Copper Sulphate

Silver Nitrate

Silver Nanoparticles

\section{Commercial compounds}

Chlorine Dioxide

Oxine (Activated)

Oxyspray

Diodine

Bacticure

Teracep

Trifilm

Trigene

Sporekil

Envirosan

Viraclean

\section{Alternative}

Allicin

Methylglyoxal
Aminoglycoside antibiotic widely used in microbiology and in limited clinical use.

Broad spectrum antibiotic used clinically to treat bacterial meningitis [141].

Tetracycline antibiotic in clinical use with some indications for veterinary and agricultural use [142].

Aminoglycoside, used in agriculture/horticulture settings in New Zealand.

Aminoglycoside antibiotic. Used to combat streptomycin resistant fire blight in USA. Used to combat fungal and bacterial infections in rice plantations. Different ribosomal binding site and resistance mechanisms to streptomycin [139].

Represents the copper salts, usually in complex formulas, used in many horticultural situations.

Soluble silver salt with known antibiotic activity but limited field use [143].

Colloidal silver suspension from VUW [144].

Tablet form, activated with water [145].

Liquid chlorine dioxide generator activated by citric acid.

Stabilised chlorine dioxide solution.

Active ingredient, Dodecylguanidine monoacetate [146].

Unknown formulation supplied by Seeka Kiwifruit Ltd

Hydrogen Peroxide $<30 \%$, Acetic acid $<10 \%$, Peracetic acid $<10 \%$.

Hydroxymethyl-2-nitro-1 3-propanediol 19.2\%, Alkyl dimethyl benzyl, Ammonium chloride 3.08\%, Formaldehyde $2.28 \%$.

Polymeric biguanide hydrochloride $<2 \%$, Alkyldimethyl benzyl ammonium chloride, (Didecyldimethyl) $<12.5 \%$, Didecylamine sulphamate $<5 \%$.

Didecyl-dimethyl ammonium chloride (quaternary ammonium compound).

Glutaraldehyde 10\%, quaternary ammonium 5\%.

Benzalkonium chloride $0.426 \%$, proprietary blend $10-30 \%$.

Garlic extract [147].

Active antimicrobial compound in Manuka honey [148]. 


\subsubsection{Results}

A subset of the inhibition curves for a range of the tested compounds are shown in figures 3.3 - 3.5. These figures represent three different types of compounds tested: classical antibiotics (oxytetracycline); commercial products (chlorine dioxide tablets); and metal ion solutions (silver nitrate). The calculated $\mathrm{IC}_{50}$ values of all compounds and mixtures that were successfully tested in this study are summarised in Table 3.2.

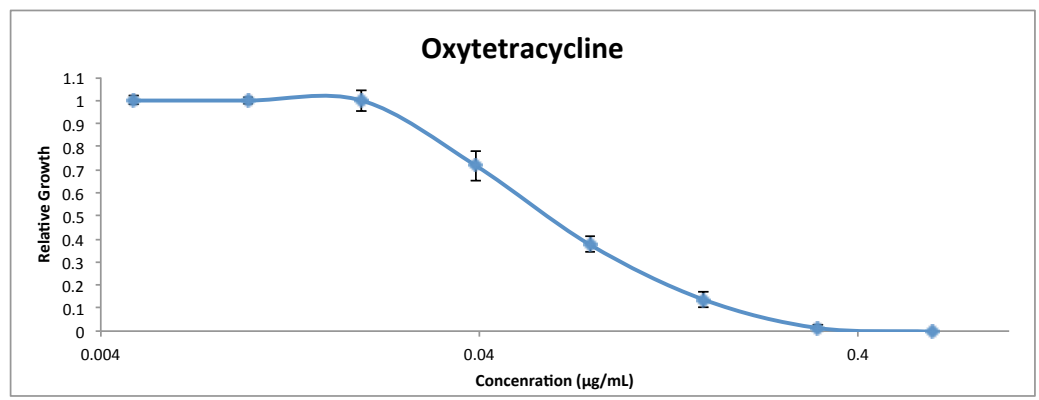

Figure 3.3: The Psa-V does response curve for the antibiotic oxytetracycline. This plot is a representative example of the dose response curve for the antibiotic class of compounds tested. On the plot, each data point represents the mean relative growth $( \pm \mathrm{SD})$ of six replicate cultures compared to non-treatment controls. The solid blue line is a continuous trend of the data points, and the reported $\mathrm{IC}_{50}$ values (Table 3.2) were calculated by fitting a four-parameter non-linear regression dose-response curve. The concentration values (X-axis) are scaled by $\log _{10}$.

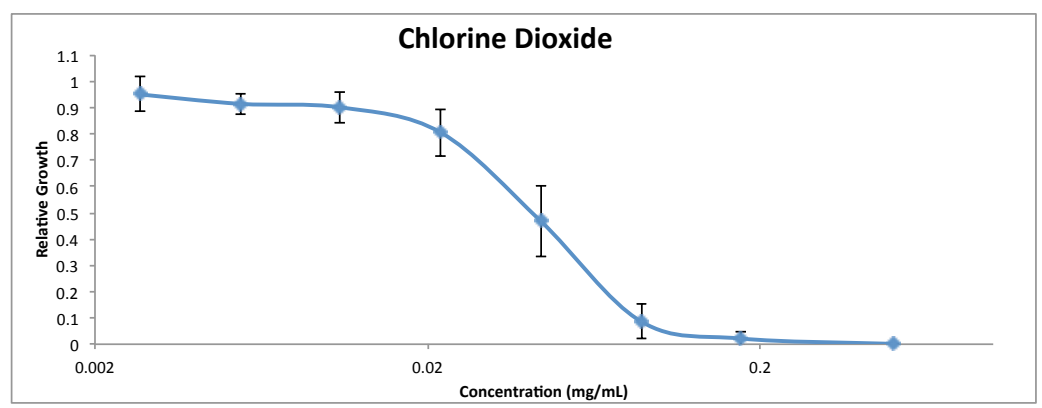

Figure 3.4: The Psa-V does response curve for chlorine dioxide (tablets). This plot is a representative example of the dose response curve for the commercial class of compounds tested. On the plot, each data point represents the mean relative growth $( \pm \mathrm{SD})$ of six replicate cultures compared to non-treatment controls. The solid blue line is a continuous trend of the data points, and the reported $\mathrm{IC}_{50}$ values (Table 3.2) were calculated by fitting a four-parameter non-linear regression dose-response curve. The concentration values (X-axis) are scaled by $\log _{10}$.

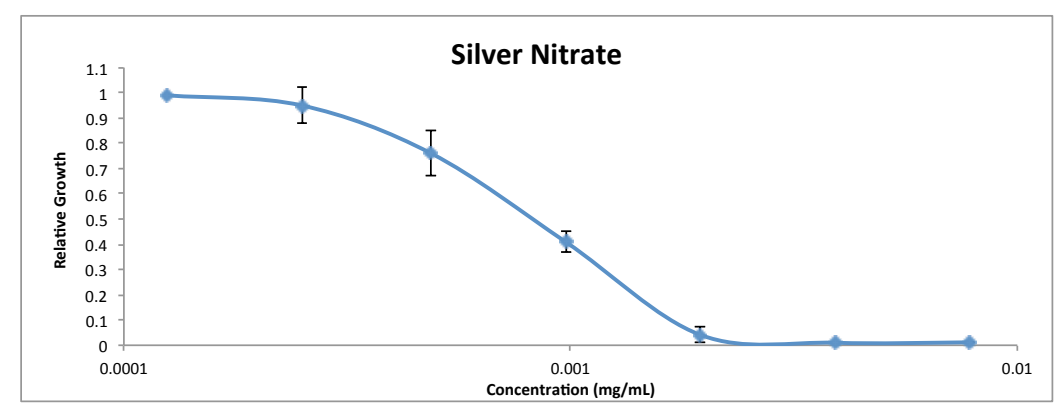

Figure 3.5: The Psa-V does response curve for silver nitrate. This plot is a representative example of the dose response curve for the metal ion class of compounds tested. On the plot, each data point represents the mean relative growth $( \pm \mathrm{SD})$ of 6 replicate cultures compared to non-treatment controls. The solid blue line is a continuous trend of the data points, and the reported $\mathrm{IC}_{50}$ values (Table 3.2) were calculated by fitting a four-parameter non-linear regression dose-response curve. The concentration values (x-axis) are scaled by $\log _{10}$. 
Table 3.2: $\mathrm{IC}_{50}$ results for tested compounds.

\begin{tabular}{|c|c|c|}
\hline \multirow[t]{2}{*}{ Compound/Formula } & \multicolumn{2}{|c|}{$\mathbf{I C}_{50}$} \\
\hline & $\begin{array}{c}\text { Concentration }(\mathrm{mg} / \mathrm{L}) \\
\text { or Dilution }(\mathrm{mL} / \mathrm{L})\end{array}$ & Molarity (where known) \\
\hline Streptomycin Sulphate & $3.80 \mathrm{mg} / \mathrm{L} \pm 0.18$ & $2.6 \mu \mathrm{M}$ \\
\hline KeyStrepto ${ }^{\circledR}$ (Streptomycin) & $3.60 \mathrm{mg} / \mathrm{L}^{1}$ & $2.5 \mu \mathrm{M}$ \\
\hline Oxytetracycline & $0.20 \mathrm{mg} / \mathrm{L} \pm 0.01$ & $0.41 \mu \mathrm{M}$ \\
\hline Kasugamycin & $17.10 \mathrm{mg} / \mathrm{L}^{1}$ & $41.2 \mu \mathrm{M}$ \\
\hline Gentamicin & $0.30 \mathrm{mg} / \mathrm{L} \pm 0.01$ & $0.64 \mu \mathrm{M}$ \\
\hline Chloramphenicol & $9.0 \mathrm{mg} / \mathrm{L} \pm 0.30$ & $28.3 \mu \mathrm{M}$ \\
\hline Ag Nanoparticles & Unknown $^{2}$ & $4.0 \mu \mathrm{M} \pm 0.70$ \\
\hline Sliver Nitrate $\left(\mathrm{AgNO}_{3}\right)$ & $1.89 \mathrm{mg} / \mathrm{L} \pm 0.45$ & $11.10 \mu \mathrm{M}$ \\
\hline Copper Sulphate $\left(\mathrm{CuSO}_{4}\right)$ & $840 \mathrm{mg} / \mathrm{L}^{1}$ & $3.3 \mathrm{mM}$ \\
\hline Chloride Dioxide & $138.0 \mathrm{mg} / \mathrm{L} \pm 15.9$ & \\
\hline Bacticure & $29.2 \mathrm{mg} / \mathrm{L} \pm 2.3$ & \\
\hline Citrox & $0.06 \mathrm{~mL} / \mathrm{L} \pm 0.0041$ & \\
\hline Oxine (Activated) & $0.06 \mathrm{~mL} / \mathrm{L} \pm 0.004$ & \\
\hline OxySpray & $0.25 \mathrm{~mL} / \mathrm{L} \pm 0.009$ & \\
\hline Diodine & $0.01 \mathrm{~mL} / \mathrm{L} \pm 0.0002$ & \\
\hline Teracep & $0.07 \mathrm{~mL} / \mathrm{L} \pm 0.020$ & \\
\hline Trifilm & $0.26 \mathrm{~mL} / \mathrm{L} \pm 0.010$ & \\
\hline Envirosan & $0.04 \mathrm{~mL} / \mathrm{L} \pm 0.001$ & \\
\hline Trigene & $0.04 \mathrm{~mL} / \mathrm{L} \pm 0.002$ & \\
\hline Sporekil & $0.02 \mathrm{~mL} / \mathrm{L} \pm 0.002$ & \\
\hline Viraclean & $1.9 \mathrm{ml} / \mathrm{L} \pm 0.180$ & \\
\hline Methylglyoxal & $0.40 \mathrm{~mL} / \mathrm{L} \pm 0.001$ & $3.2 \mathrm{mM}$ \\
\hline Allicin & $6.0 \mathrm{~mL} / \mathrm{L} \pm 0.2$ & \\
\hline
\end{tabular}

Error is reported as $\pm \mathrm{SE} \log _{10} \mathrm{IC}_{50}$ as the concentration data points are symmetrical on the $\log _{10}$ scale. ${ }^{1}$ Error is undefined. ${ }^{2}$ This solution contained compounds of unknown molecular weight, the molarity was calculated from data supplied by the provider.

\subsubsection{Chloramphenicol and gentamicin activity}

Two of the antibiotics tested above, chloramphenicol and gentamicin, were included in the collection primarily because they were of particular interest to the intended gene knockout work described in the following chapter. This was because the Ackerley lab possesses two counter-selectable suicide plasmids that have been designed for gene knockout studies in Pseudomonas spp.; one of these vectors (pDM4) contains a chloramphenicol resistance marker gene, and the other (pEX18Gm) a gentamicin resistance marker gene. To justify if either (or both) vector might be appropriate for use in Psa-V, each of the vector relevant antibiotics were tested in the optimised $\mathrm{IC}_{50}$ assay, despite these compounds being inappropriate candidates for field antibiotic spray trials due to their current use as clinical antibiotics in human health care [149-151]. 
Initial data from the assay concluded that both chloramphenicol $\left(\mathrm{IC}_{50}=5.6 \mu \mathrm{M}\right)$ and gentamicin $\left(\mathrm{IC}_{50}=2.7 \mu \mathrm{M}\right)$ had reproducible antimicrobial effects against Psa-V, yielding inhibition curves comparable to other antibiotics tested and suggesting either vector would be suitable for use in the knockout study.

\subsubsection{In vitro activity of established field use antibiotics}

In terms of molarity of active ingredient the $\mathrm{IC}_{50}$ of streptomycin sulphate (purchased from Sigma-Aldrich at a purity of $\geq 98 \%$ ) and KeyStrepto ${ }^{\circledR}$ (Key Industries, streptomycin concentration $=17 \%$ ) were found to be essentially identical (Table 3.2), indicating that the formulation of KeyStrepto ${ }^{\circledR}$ does not reduce its potency. Both forms of streptomycin yielded $\mathrm{IC}_{50}$ results at least one order of magnitude lower than kasugamycin (i.e. more effective), a finding comparable with other published results $[152,153]$. The recommended application rates reflect heightened potency of KeyStrepto ${ }^{\circledR}$ over Kasumin ${ }^{\circledR}$. Compulsory KVH guidelines for antibiotic use state that KeyStrepto ${ }^{\circledR}$ must be applied at a concentration equivalent to $\sim 70 \mu \mathrm{M}$ of streptomycin, whereas the application concentration for Kasumin ${ }^{\circledR}$, equates to $\sim 230 \mu \mathrm{M}$ of kasugamycin.

\subsection{A novel chemical inhibitor system of Psa-V}

During the course of this research, an unrelated project in the Ackerley lab, which was using the chemical niclosamide as a positive selection agent for nitroreductase enzymes in E. coli, found that cells lacking endogenous nitroreductases were profoundly sensitive to this compound, but only when they had the gene encoding the TolC efflux pump deleted from the chromosome. This result suggested that niclosamide may be, when used in conjunction with an efflux pump inhibitor, repurposed as a novel antimicrobial agent against bacteria that lack substantial nitroreductase activity. Because pilot studies suggested that Psa-V might fall into this category and in the interest of possibly expanding the repertoire of control options, research efforts were turned towards further defining this novel system.

\subsubsection{Niclosamide}

Niclosamide is a halogenated salicylanilide

(Fig.3.6) that is used in a variety of applications, mainly as a molluscicide, in an agricultural, veterinary and human health context. As such the compound is commercially available (e.g. Bayluscide ${ }^{\circledR}$ - which is marketed to control snail populations in fish ponds [154]). In human health settings, niclosamide is an important anthelmintic drug for the treatment

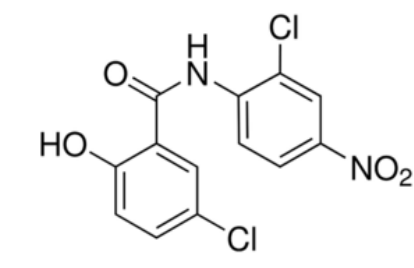

Figure 3.6: Niclosamide $\left(2^{\prime}, 5-\right.$ dichloro-4'-nitrosalicylanilide) of tapeworms and is also found to be useful in reducing prevalence of the tropical parasitic disease schistosomiasis by destruction of its intermediate host, the snail Biomphalaria glabrata. Because of these applications, there have been many pharmacological trials of niclosamide and it is considered safe for use in humans [155]. 
Recent studies have found niclosamide to be a quorum-sensing inhibitor in $P$. aeruginosa, i.e. it can reduce the virulence and motility of this species by suppressing the production of quorum sensing molecules [156]. These points are considered relevant as they indicate low toxicity to humans, which could potentially facilitate registration of the product for use in New Zealand kiwifruit orchards.

\subsubsection{Preliminary evaluation of nitroreductase activity in Psa-V}

Preliminary BLAST (tblastn; AA query, DNA database) searches querying the translated E. coli nitroreductases genes $n f s A$ and $n f s B$, the two most active families of bacterial nitroreductase identified to date [157], against the Psa-V genome returned two hits of low amino acid pairwise identity (37\% identical sites with $98.8 \%$ query coverage and $24 \%$ identical sites with $72.9 \%$ query coverage) for the translated $n f s A$ query and no hits of any significant identity for translated $n f s B$ query. A pairwise amino acid alignment of the hit with the highest degree of homology is shown in Figure 3.7. The results of the BLAST searches at the amino acid and nucleotide level (not shown) with these particular nitroreductases did not present significant evidence for functional homology. However, the Psa-V Nz13 complete genome does contains five different annotated genes that all have $>90 \%$ homology to genes putatively annotated as nitroreductases, or nitroreductase family proteins, in other $P$. syringae species.

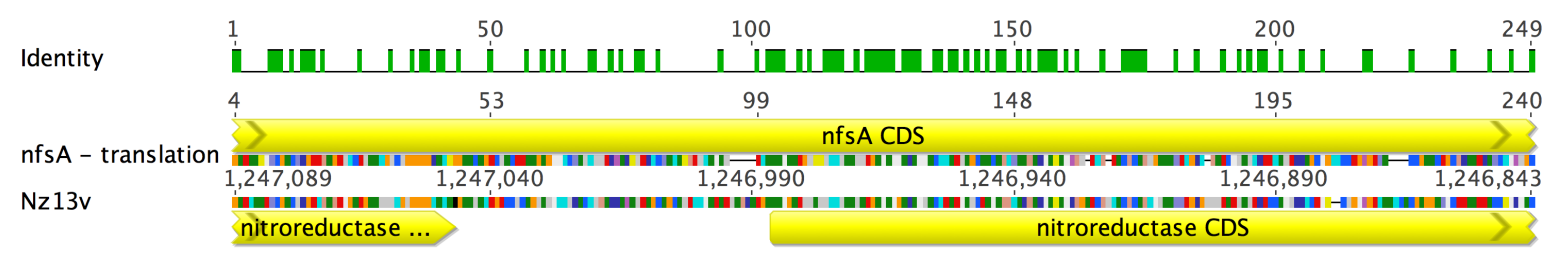

Figure 3.7: Graphical output of the amino-acid pairwise alignment view from the best (highest pairwise identity) tblastn hit of the translated $n f s A$ gene against the Psa-V genome. This hit has $98.8 \%$ query coverage and $37.3 \%$ identical sites. Pairwise identity is displayed above the alignment as a green bar, and the green regions represent identity and breaks in the bar represent mismatches or gaps. The $n f s A$ translation query does not directly map to any predicted gene translations in the Psa-V genome.

Empirical evidence of the level of endogenous nitroreductase activity of Psa-V was sought. The $\mathrm{IC}_{50}$ assay described in section 2.13 was employed to compare the sensitivity of wildtype Psa-V and E. coli to nitrofurantoin, a prodrug antibiotic that is activated by nitro-reduction $[158,159]$, as a generic test for nitroreductase activity. This test found that nitrofurantoin was not effective in killing Psa-V relative to $E$. coli $\left(\mathrm{IC}_{50}\right.$ of $47.2 \mu \mathrm{M}$ for $E$. coli and $1097 \mu \mathrm{M}$ for Psa-V; Fig. 3.8), suggesting that Psa-V does not express any highly active nitroreductase enzymes. This data in turn suggested that Psa-V might not readily be able to detoxify niclosamide by nitroreduction. 


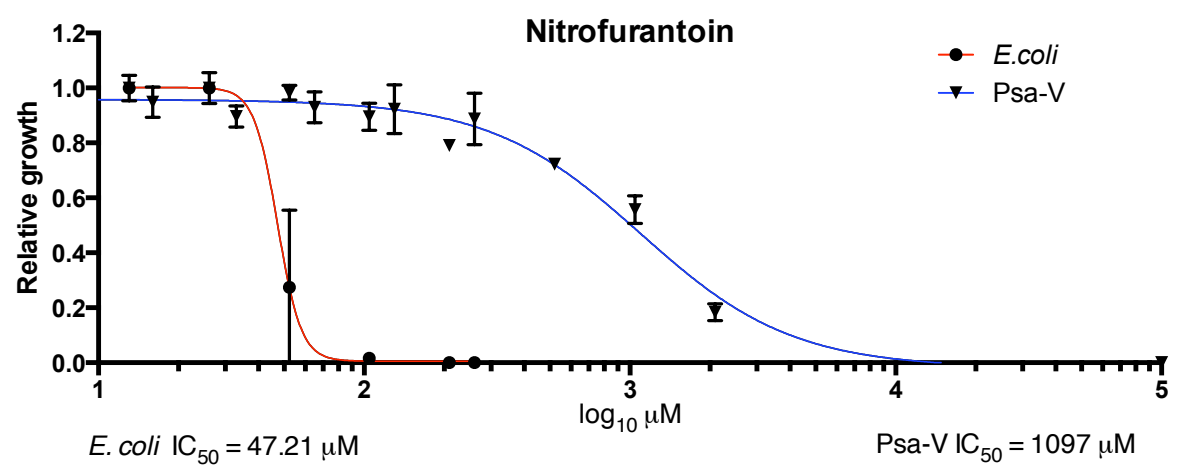

Figure 3.8: Variable slope symmetrical sigmoidal dose response curves fitted to the data points of Psa-V (blue line) and E.coli (red line) growth inhibition caused by the antibiotic prodrug nitrofurantoin. The concentration of nitrofurantoin is shown in $\log _{10}$ scale. These two slopes were used to calculate the $\mathrm{IC}_{50}$ s of nitrofurantoin for Psa-V and E.coli. The assay was set up and calculations were run as per previous $\mathrm{IC}_{50}$ assays, the data points are the mean of six replicates and data point error bars are $1 \mathrm{STD}$ of the mean. $\mathrm{IC}_{50}$ Error: $\left( \pm \mathrm{SE} \log _{10} \mathrm{IC}_{50}\right)$ Psa-V $=0.056, E$. coli $=0.029$.

\subsubsection{Efflux pumps and efflux pump inhibitors}

As suggested in section 3.3, in addition to a lack of protective nitroreductase activity, bacterial cells must also lack protection from effective multidrug efflux pumps for niclosamide to be highly toxic. Efflux pumps are membrane-spanning transporter proteins with a wide range of substrates and they confer resistance to many antibiotics in Gram negative bacteria by extruding the cytotoxic compounds, and other substrates, across both membranes and out of the cell. The expression of multidrug efflux pumps in $P$. aeruginosa is known to result in phenotypes resistant to virtually all classes of clinically relevant antibiotics [160]. As drug efflux is an important mechanism of bacterial drug resistance, efflux pump inhibitors have been developed to combat the occurrence of multidrug resistance in pathogenic strains. In previous studies, efflux pump inhibitors exhibited the ability to potentiate antimicrobial compounds and reverse resistant phenotypes [161].

A lead compound to come from this research is phenylalanyl-arginyl-beta-naphthylamide $(\mathrm{PA} \beta \mathrm{N})$, a low molecular weight dipeptide amide. PA $\beta \mathrm{N}$ has low levels of intrinsic antimicrobial activity, but it is a powerful broad spectrum bacterial efflux pump inhibitor that can inhibit the functions of the TolC and MexE efflux pumps and it has been shown to increase the sensitivity of even resistant bacterial strains, including pseudomonads, to several classes of antibiotics and antimicrobial compounds [162-164]. Both tolC and mexE like genes are found in Psa-V (Fig. 3.9). Although present in the Psa-V genome, as it was unknown whether or not Psa-V expresses these efflux pumps to effectively export niclosamide.

\subsubsection{Inhibition of Psa- $\mathrm{V}$ by niclosamide in combination with the efflux pump inhibitor PA $\beta N$}

In this study niclosamide alone was found to mildly inhibit the growth of Psa-V in rich media. When niclosamide was combined with the efflux pump inhibitor PA $\beta N$, a very strong inhibitory effect against Psa-V was seen.

To test the efficacy of niclosamide $\pm \mathrm{PA} \beta \mathrm{N}$ in inhibiting Psa-V, a dual axis concentration assay was designed for a 96 well plate using serial dilutions of each compound running 


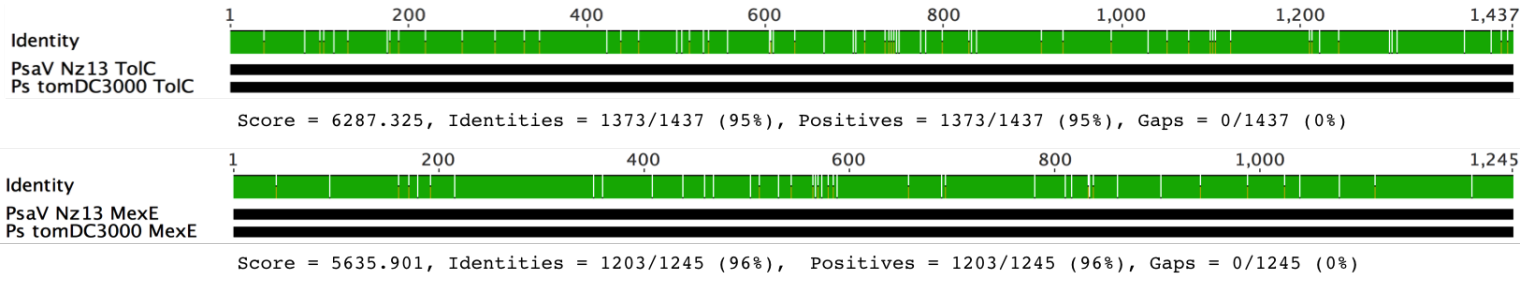

Figure 3.9: Full gene pairwise nucleotide alignments of two multidrug efflux pumps from Psa-V Nz13 with their closest BLAST hits from the NCBI database: A) Nucleotide alignment of tolC from Psa-V Nz13 and P.syringae pv. tomato DC3000. B) Nucleotide alignment of mexE from Psa-V Nz13 and P.syringae pv. tomato DC3000.

The solid black bars represent the nucleotide sequences being aligned with the sequence name to the left. The green bar above the alignments shows the pairwise identity, and the green regions represent identity and the breaks represent mismatches. This global alignment (Needleman-Wunsch [165]) used a cost matrix set at $93 \%$ with an open gap penalty of 12 and an extension gap penalty of 3 . The alignments score data is displayed below each alignment.

perpendicular to each other. This was achieved by establishing the concentration gradients of each compound in separate plates then combining these plates to create a test plate with the desired array of concentrations. A standardised culture of Psa- $\mathrm{V}$ was challenged in the test plate and incubated for 16 hours. This experiment was designed to establish which combination of the lowest concentrations of each drug was needed to effectively inhibit Psa-V. A heat map showing the average results of three biological replicates challenged in this way is pictured below (Fig. 3.10).

\begin{tabular}{|c|c|c|c|c|c|c|c|c|c|}
\hline $\begin{array}{l}\text { Negative } \\
\text { treatment }\end{array}$ & \multicolumn{8}{|c|}{ Psa-V \% Growth ( $\pm 12.5 \%)$} & \multirow{2}{*}{$\begin{array}{c}\mu \mathrm{M} \text { [NCS] } \\
200 \\
\end{array}$} \\
\hline $100 \%$ & $0 \%$ & $0 \%$ & $20 \%$ & $42 \%$ & $50 \%$ & $52 \%$ & $54 \%$ & $60 \%$ & \\
\hline $100 \%$ & $0 \%$ & $0 \%$ & $24 \%$ & $42 \%$ & $53 \%$ & $51 \%$ & $58 \%$ & $57 \%$ & 100 \\
\hline $100 \%$ & $0 \%$ & $0 \%$ & $30 \%$ & $57 \%$ & $66 \%$ & $62 \%$ & $61 \%$ & $63 \%$ & 50 \\
\hline $100 \%$ & $0 \%$ & $0 \%$ & $33 \%$ & $68 \%$ & $71 \%$ & $68 \%$ & $62 \%$ & $69 \%$ & 25 \\
\hline $100 \%$ & $1 \%$ & $34 \%$ & $65 \%$ & $76 \%$ & $83 \%$ & $79 \%$ & $83 \%$ & $82 \%$ & 13 \\
\hline $100 \%$ & $35 \%$ & $48 \%$ & $77 \%$ & $88 \%$ & $88 \%$ & $86 \%$ & $92 \%$ & $89 \%$ & 6 \\
\hline 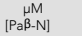 & 50 & 25 & 13 & 6 & 3 & 2 & 1 & 0.5 & 0 \\
\hline
\end{tabular}

Figure 3.10: Results from dual axial concentration gradient challenge using niclosamide and PA $\beta N$ against Psa-V. In this figure the relative growth of Psa- $\mathrm{V}$ is shown as a mean percentage value of the untreated controls for three replicates (Error: \pm mean SD), shown in $\mu \mathrm{M}$ are the concentrations corresponding to the rows and columns for niclosamide and PAßN respectively (blue). The percentage values shown in the leftmost column represent the untreated control wells containing only Psa-V and growth media.

An accurate $\mathrm{IC}_{50}$ of niclosamide in isolation, against Psa-V, could not be obtained as the compound becomes insoluble in aqueous growth media before an inhibitory concentration, required for this calculation, can be reached. For PAßN in isolation, the $\mathrm{IC}_{50}$ against Psa-V was calculated at $20 \mu \mathrm{M}$.

The results in the above figure show that in combination the effect of Psa-V growth inhibition is more than the additive effect of each drug in isolation. At a concentration of 25 $\mu \mathrm{M}$ each in combination these chemicals killed $100 \%$ of the cells (i.e no growth detected 
after 16 hours incubation and zero colonies recovered when the media from the test wells was plated on rich media). The concentrations needed to give $100 \%$ cell death using this combination of drugs is comparable with that of the antibiotics tested previously (section 3.2.4). Usually $\mathrm{IC}_{50} \mathrm{~s}$ are used to compare efficacy but this was not done here for two reasons, it was not possible to establish the $\mathrm{IC}_{50}$ of niclosamide and as the supply of PAßNwas limited the lowest possible effective concentration (i.e. $100 \%$ cell death) of this component was of interest. However, the results of this experiment suggest that niclosamide in combination with PA $\beta N$ might have utility as a novel anti-Psa-V agent.

\subsection{Development of antibiotic resistance in Psa-V}

Although there are many examples (in the literature) of $P$. syringae developing antibiotic resistance, especially to streptomycin [166-168], the ability of the epidemic strain of Psa-V to become resistance to the antibiotics that are now used in the field (streptomycin and kasugamycin) has not been fully investigated. The general mechanisms of action of both of these antibiotics are well understood and several possible resistance mechanisms to these drugs are known [169]. An inquiry into the antibiotic resistance potential of Psa- $\mathrm{V}$ is relevant to the current practices carried out in kiwifruit orchards and results here may guide future practices to prevent or minimise possible antibiotic resistance occurring in the field.

\subsubsection{Antibiotic mechanisms and resistance}

Streptomycin and kasugamycin both belong to the aminoglycoside family of antibiotics. These molecules produce their bactericidal effects by binding to sites along the mRNA binding channel in the $30 \mathrm{~S}$ ribosomal subunit [170]. The binding of the drugs in the subunit diminishes ribosomal proofreading capabilities by stabilising near-cognate tRNAs to the mRNA being read by the ribosome, leading to mistranslation. The secondary effects of this antibiotic induced translation infidelity leads to cell death [171]. Although in the same structural family and sharing similar modes of protein synthesis inhibition, these two antibiotics each bind to different target sites within the mRNA binding channel of the 30S subunit. Resistance to each of these drugs has been reported in various strains of bacteria, but importantly the mode of resistance is different for each.

Bacterial resistance mechanisms to streptomycin include production of aminoglycoside-modifying enzymes which can be carried on plasmids, chromosomal mutations that lead to modification of the target site, and prevention of streptomycin access to the target site by permeability barriers. Common mechanisms of transferable aminoglycoside resistance are via conjugatible plasmids that carry genes encoding for aminoglycoside modifying enzymes that acetylate, phosphorylate or adenylylate the antibiotics $[172,173]$. A comprehensive review of these enzymes is presented in [171]. Of particular relevance to Psa-V, StrA and StrB are examples of specific streptomycin modifying enzymes aminoglycoside-3-phosphotransferase $\left[\mathrm{APH}\left(3^{\prime}\right)-\mathrm{Ib}\right]$ and aminoglycoside-6-phosphotransferase [APH(6)-Id] [174] that are reportedly found in strains of Psa isolated from Japan [168]. 
Development of streptomycin resistance in pseudomonads may also arise in a plasmid independent fashion, by a spontaneous single nucleotide polymorphism (SNP) within the rpsL gene. The product of this gene is the ribosome associated protein S12. S12 is important for ribosomal fidelity as it allows for changes between the different yet specific ribosomal conformations required for faithful protein translation. An amino acid change caused by the rpsL SNP, resulting in the substitution of the wildtype lysine residue by an arginine, a threonine or an asparagine at codon 43 [175], destabilises the binding of the S12 enzyme to the ribosome. Streptomycin, binding to a similar site, constricts the ribosome to only one of the conformations allowed by the interaction of the ribosome with S12, but this streptomycin-induced conformational restriction is not exhibited in a strain carrying the rpsL SNP. It is thought that the mutant S12's relaxed binding properties allow all of the needed ribosomal confirmations to exist even in the presence of streptomycin [176]. Across several studies, this is found to be by far the most common of a small number of possible chromosomal mutations leading to streptomycin resistance [177-183]. This mutation is shown to give very high levels of streptomycin resistance and has been reported in strains of non-epidemic Psa isolated from Japan [168].

High-level kasugamycin resistance (maximal $>64$ fold over wildtype) in $E$.coli is conferred by several possible point mutations in the 16S RNA gene that are able to function independently [184]. The three most important positions identified in the 16S RNA gene for kasugamycin resistance mutations, using numbering from E. coli, are A794, G926 and A1519. The first two sites were found to be directly involved with kasugamycin binding. Binding of the drug protects these residues from chemical modification and these binding positions were subsequently elucidated by crystallographic analysis of the ribosome with the drug bound [185]. Kasugamycin cannot bind when either of these sites are mutated [170]. The remaining site (A1519) makes up part of the conserved ksg1 site of the 16S subunit and a mutation here gives the lowest level of resistance of the three identified ( $\sim 32$ fold). The ksg1 site is one of the targets of an adenine dimethylase (KsgA) and the lack of post-transcriptional modification by this methylase at the site is well known to produce modest kasugamycin resistance, thus a functional copy of $k s g A$ is required for kasugamycin to effectively inhibit translation in an otherwise normal genetic background [186, 187]. Furthermore, site-directed mutagenesis of all three of these highly conserved [188] nucleotide positions showed that practically any mutation was allowed and would confer kasugamycin resistance, but the mutations did not significantly reduce bacterial doubling time [184]. Within the genomes of E. coli and Psa-V there are several copies ( 7 and 5 respectively) of the 16S RNA gene, so significant kasugamycin resistance by the mechanism of point mutation of this gene is unlikely as every copy would have to be mutated at the same position to give full resistance. Resistance due to dysfunctional $k s g A$ may be more likely but dysfunction or deletion of this universally conserved gene impairs ribosomal biogenesis and is shown to be highly deleterious to cell growth [189]. This is compared to the streptomycin resistance scenario of single point mutations in the single copy rpsL gene giving full resistance with, in some known cases, no fitness cost. These chromosomal mutations cannot be transferred to Psa-V by a plasmid. Drug-modifying enzymes that can protect against kasugamycin have been described. For example, a kasugamycin specific 
2'-N-acetyltransferase gene aac(2')-IIa confers resistance to this drug by acetylating the 2' amino residue [190]. The gene has been found on pathogenicity islands in the rice pathogens Burkholderia glumae and Acidovorax avenae subsp. avenae. These protective enzymes could plausibly be expressed from genes carried on a plasmid or introduced by horizontal gene transfer. In summary, it would appear that spontaneous resistance to kasugamycin is much harder to evolve than resistance to streptomycin in $E$. coli because of the extremely low probability or fitness cost involved with kasugamycin resistance mutations.

The observation of spontaneous chloramphenicol resistance developing at frequently to high levels of this antibiotic in Psa-V during early knockout experiments, which used this antibiotic as a selectable marker (see section 3.2.5), and the indications from the literature of frequent streptomycin resistance occurring in other $P$. syringae pathovar prompted an experimental investigation into the possibility of spontaneous streptomycin and/or kasugamycin resistance arising in Psa-V. It was hypothesised that streptomycin resistance would be induced more readily than resistance to kasugamycin in Psa-V because kasugamycin resistance should require many separate mutations to occur within the same cell due to multiple copies of the 16S RNA gene in the chromosome.

\subsubsection{Spontaneous streptomycin and kasugamycin resistance in Psa-V}

Initial laboratory experiments were designed to select for spontaneous streptomycin resistance in Psa-V. Spontaneous resistance is due to random mutations in chromosomal genes that reduce or eliminate sensitivity to a particular antibiotic without horizontal gene transfer or plasmid uptake [191]. Streptomycin resistant strains were developed by serially passaging wildtype Psa-V clones in rich growth media (liquid or agar) supplemented with an incrementally increasing streptomycin concentration at each subculture step until a strongly resistant phenotype was isolated. This experiment was replicated using kasugamycin as the selection pressure, but no detectably resistant mutants were isolated in the allowed timeframe of this study. Of note, the streptomycin resistant strains did not possess any cross-resistance to kasugamycin. This is consistent with the hypothesis above.

\subsubsection{Induced streptomycin resistance results}

During the process of subculturing Psa-V across incrementally increasing concentrations of streptomycin (section 2.12) it was consistently observed that resistance would develop at an intermediate concentration of streptomycin, to a level that allowed the resistant isolate to be clonally propagated directly onto media containing very high streptomycin concentrations without the need for continuing the incremental concentration increases. Three streptomycin resistant isolates of Psa-V (Psa-V Strep ${ }^{R}$ 1-3) were generated in independent experiments, with all replicates developing equivalent resistance to streptomycin $\left(\mathrm{IC}_{50} \mathrm{~s}\right.$ of $\approx 2 \mathrm{mM}$; Fig. 3.11). Colonies of Psa-V Strep ${ }^{\mathrm{R}} 1-3$ were routinely maintained at $70 \mu \mathrm{M}$ streptomycin, and this is an $\sim 30$ fold concentration increase over the wildtype Psa-V streptomycin $\mathrm{IC}_{50}$. To ensure no inadvertent contamination of the cultures with other bacteria, the resistant strains were confirmed as PsaV by colony PCR using two sets of PsaV specific primers, PsaV F/R and HopZ5 F/R. 


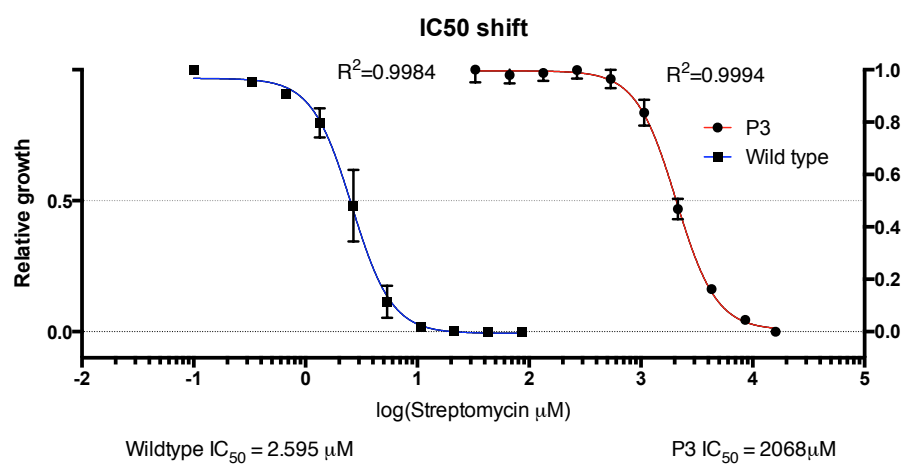

Figure 3.11: Plot of the streptomycin $\mathrm{IC}_{50}$ shift of wildtype and a streptomycin resistant strain of Psa-V (P3). Data points are the mean of six replicates and error bars are \pm 1 STD, the lines fitted to the data points, from which the $\mathrm{IC}_{50}$ data is derived, is a global non-linear regression variable slope four-parameter dose-response curve. $\mathrm{R}^{2}$ values for each of these fits are reported next to the curves. The SE of the $\mathrm{IC}_{50}$ values, reported as $\pm \log _{10} \mathrm{IC}_{50}$, are 0.010 for $\mathrm{P} 3$ and 0.016 for wildtype. The ratio of the $\mathrm{IC}_{50}$ s is $797: 1$.

\subsubsection{A chromosomal mutation in $r p s L$ causes streptomycin resistance in Psa-V}

Reasoning that the in vitro culturing process did not allow horizontal gene transfer from other bacteria, it was hypothesised that one or more chromosomal mutations were responsible for the observed phenotype. Moreover, it was assumed that one chromosomal mutation in particular was capable of conferring substantial streptomycin resistance, consistent with the clones recovered at intermediate challenge concentrations now being resistant to very high levels of streptomycin.

As streptomycin resistance in other bacteria has been associated with a SNP (in codon 43) of the rpsL gene, this gene was amplified from all three of the independently-generated Psa-V Strep ${ }^{\mathrm{R}}$ isolates and from the ancestral wildtype colonies from which they were developed. The PCR product was then Sanger sequenced in both directions to screen for the expected mutation.

A quality-based consensus sequence for each sample was produced by a de novo assembly of the respective forward and reverse reads of quality trimmed chromatogram data (error probability threshold: 0.05). As the chromatogram data used here, supplied by Macrogen sequencing services, contains base calling confidence values (Phred scores [192]) the expected number of base call errors in these constructed consensus sequences can be calculated. For all wildtype samples the final expected base calling error for the area of sequence containing the rpsL open reading frame was 0.00 , and for the three replicate Psa-V Strep ${ }^{\mathrm{R}}$ samples it was $0.00(\mathrm{P} 3), 0.02$ (P2), and 1.48 (P1). This was calculated by converting the confidence score for each base call into the error probability and summing across the region of sequence being analysed. From this data it was expected that the P1 sequence may contain a small fraction of errors. There were in fact three bases between nucleotide positions 50 to 100 in the $\mathrm{P} 1$ sequence, which did not align with the wildtype or the P2 and P3 sequences. An inspection of the P1 consensus sequence showed that these individual positions had very a low base call confidence $(<\mathrm{Q} 20)$. The positions were thus altered to match the expected nucleotide found in the three other sequences aligned here to avoid confusion when viewing the graphical output. 
The sequences of all wildtype rpsL genes from the parental biological replicates were identical to each other and aligned perfectly with the published sequence of this gene (not shown). To examine for sequence changes to rpsL in the resistant mutants, the nucleotide sequences of $r p s L$ from the three Psa-V Strep ${ }^{\mathrm{R}}$ strains were aligned to the published rpsL wildtype sequence, but they did not carry a SNP at nucleotide position 128 in codon 43 , which was found to be the most common mutation giving rise to streptomycin resistance in multiple and diverse strains of bacteria including E. amylovora, E. coli, Mycobacterium tuberculosis, P. marginalis and pre-2008 P.syringae pv.actinidiae. [168, 178-181, 193]. However, this alignment showed all three resistant clones carried an identical SNP $(A \rightarrow G)$ at nucleotide position 263 in codon 88 . This mutation causes a lysine residue to be substituted to an arginine (K88R), with the corresponding codon being changed from AAA to AGA (Figs. $3.12 \&$ 3.13). Interestingly, the observed nucleotide and resulting amino acid change at codon 88 is the same (i.e. AAA to AGA / K to R) as the change that had previously been observed with high frequency at codon 43 [178-181]. Molecular modelling using the available crystallographic data of S12 from E. coli [194], and the positions of the homologous residues (K43 and K87 in this model), are found near the apices of loops protruding from the main fold of this small protein and are separated by less than $7 \AA$. The close proximity and identity of these residues would allow for a functional relationship, which is supported by the associated phenotype change seen when either one is substituted to arginine (Fig. 3.14).

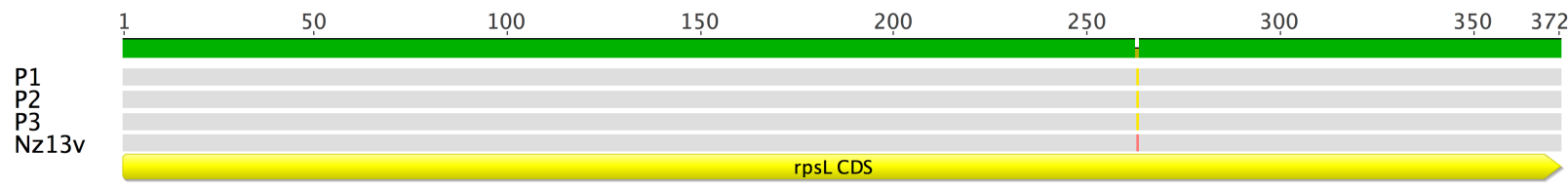

Figure 3.12: A multiple sequence alignment of $r p s L$ from the three independently generated spontaneous streptomycin resistant clones of Psa-V against the published wildtype gene sequence. P1, P2 and P3 are the sequences from the streptomycin resistant samples, Nz13v is the published rpsL sequence of wildtype Psa-V. The SNP is highlighted on the sequences with the base present in that sequence colour coded as Yellow $=\mathrm{G}$ and Pink $=\mathrm{A}$.

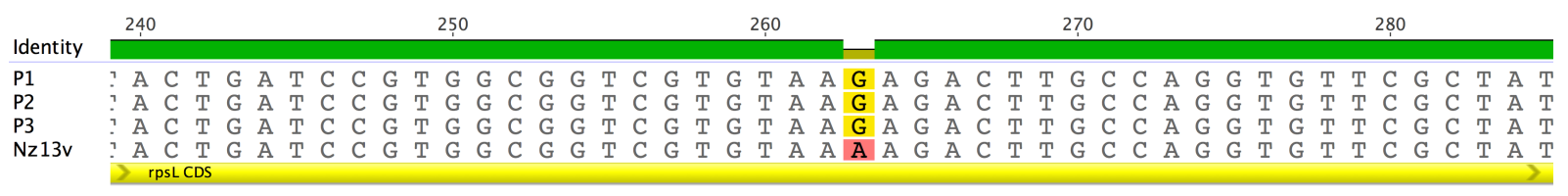

Figure 3.13: An expanded view of the rpsL alignment to show the region containing the SNP common to all three streptomycin resistant strains. The SNP is highlighted on the sequences with the base present in that sequence colour coded as Yellow $=\mathrm{G}$ and Pink $=\mathrm{A}$. 


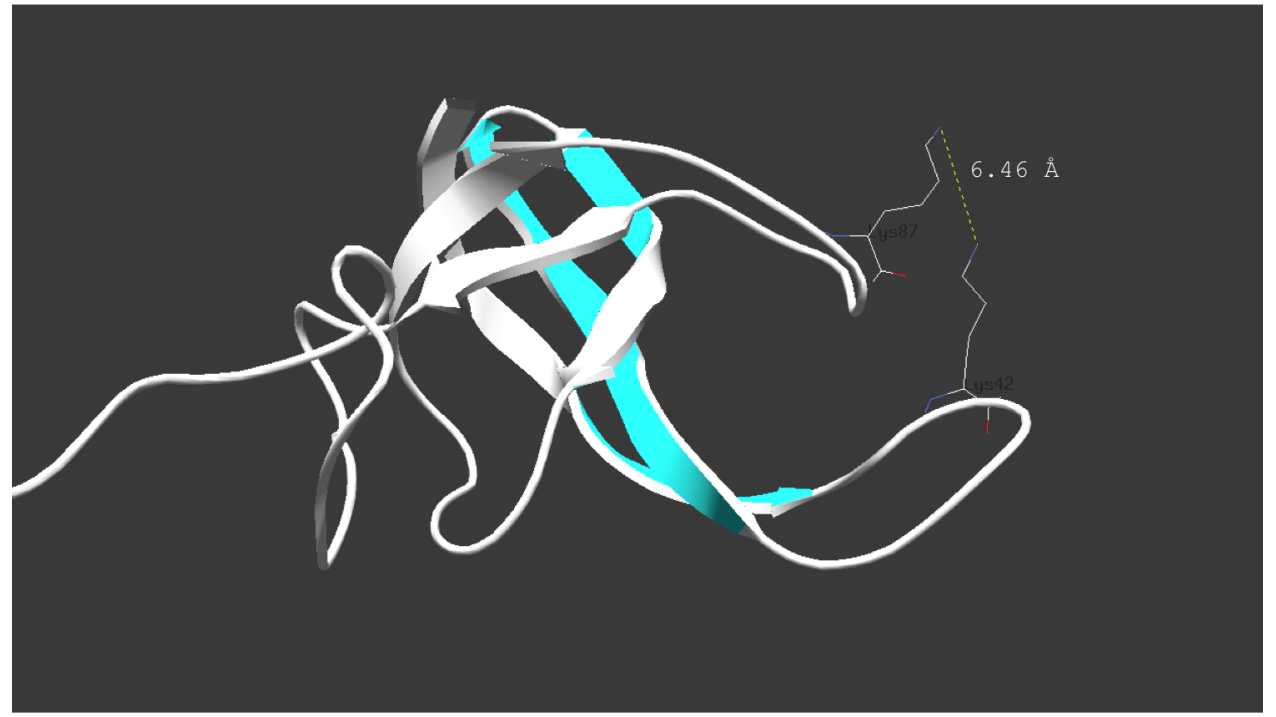

Figure 3.14: A ribbon model of residues 24 to 104 (124 total) of the $\mathrm{S} 12$ protein from E. coli showing the side chains of the lysine residues at positions 42 and 87 , and the distance between their $\varepsilon$-amino groups in angstroms.

\subsection{Fitness burden of streptomycin resistance}

Because the emergence and spread of streptomycin resistance in the field would pose a serious threat to a major component of current Psa-V control, developing strategies to keep resistance scenarios in check is of critical importance. To develop useful strategies, it is helpful to have an understanding of the dynamic between streptomycin resistance and reproductive fitness, as the occurrence and persistence of antibiotic resistance has been related to the volume and patterns of antibiotic use [195]. It has been observed that the development of resistance to antibiotics generally burdens bacterial fitness, and the effect of the burden can present itself via several biological consequences, and is typically measured as a reduction in growth rate, virulence or transmission. A optimistic interpretation of this general concept would be the possible reversal of resistance if the antibiotic selection pressure is removed [196]. There are however known resistance mutations that have no measurable fitness costs [197]. Thus, understanding how well a resistant strain performs competitively outside selective pressure may inform practices regarding antibiotic use [193]. These practices may be intended to prohibit the occurrence of resistance or to recover from resistance should it arise. They will be determined in part by the interaction between resistance and fitness as the fitness cost is a crucial parameter in determining the persistence or potential reversibility of resistance $[193,198]$. In the work described here, the competitive performance of streptomycin-sensitive and -resistant Psa-V was investigated both in vitro and in planta. It was predicted that at least three scenarios were possible. First, the mutation affording antibiotic resistance might incur an irrecoverable fitness cost, slowing the reproductive rate of the strain and thus reducing its competitive fitness. This outcome may be expected as the resistance point mutation in this case causes structural modification of streptomycin's target, the ribosome, a fundamentally important structure. Consistent with this scenario, an in vitro isolate of Borrelia burgdorferi carrying a streptomycin resistance mutation $(\mathrm{K} 88 \mathrm{E})$ in $r p s L$, equivalent to the mutation of the 
streptomycin resistant strain of Psa-V, was unable to be isolated from a co-culture competition assay after 100 generations [197]. This finding supporting the possibility of an rpsL mutation carrying a fitness cost. Secondly, the resistance mutation might cause no discernible fitness burden, allowing resistance to persist in the population outside the selective pressure of antibiotics. This is a possible outcome as resistance mutations which incur very low or no detectable fitness cost arise frequently in clinical isolates, and the same resistance mutations are commonly found to arise in in vitro studies [196, 199]. Also, in Salmonella typhimurium a no cost resistance mutation in the 42 nd codon (K42R) of rpsL is responsible for resistance to high concentrations of streptomycin [200]. These findings imply that the cost of resistance determines the emergence and survival rate of the strain carrying the resistance mutation [183] and that certain mutations in rpsL may not cause a fitness burden. The third case considered to be a possible mechanism in these competitive assays was the resistance-inducing point mutation might initially cause a physiological penalty. But this could be offset by additional compensatory mutations acquired in subsequent generations, recovering fitness to wildtype levels, and then enriched by the bottleneck of subculturing. Although it was beyond the scope of this study to elucidate the occurrence and nature of such mutations it is mentioned here, as this system of compensation has been observed in other related bacterial species carrying similar streptomycin resistant $r p s L$ point mutations [193, 200, 201].

\subsubsection{In vitro competition assay design}

It was hypothesised that the initial recovery and growth rates of the resistant strains would be retarded in comparison that of the wildtype strain when cultured in optimal laboratory conditions. To measure the relative fitness between this pair of clonally related susceptible and resistant strains an in vitro co-culture competition experiment was set up. This experiment was designed such that the two strains were inoculated together at the same density, using an initial inoculum of a predetermined optical density value measured at $\lambda=$ $600 \mathrm{~nm}$, into a single tube containing a measured volume of rich growth media, and a sample of this was taken to calculate the initial number of streptomycin resistant vs sensitive colony forming units at day 0 . The culture was incubated until the growth media was saturated with cells (24 hours). A representative sample of this saturated culture was then taken to inoculate another measured volume of fresh growth media, and an aliquot of this was used to measure day 1 colony forming units.

In laboratory studies, the bacterial fitness between otherwise isogenic strains is usually determined by such competition experiments, although interpretation of the outcome must be approached with caution as these results may not always translate to the finding in the intrinsically varied conditions of real-world systems [202, 203]. This is especially relevant when no fitness difference is seen in the in vitro experiment as any burden may not be pronounced under laboratory conditions. However strains that do present a fitness burden in vitro generally communicate this burden to in vivo conditions [193]. 


\subsubsection{In vitro competition assay results}

No statistically significant depletion of streptomycin resistant Psa-V CFUs were apparent in a co-inoculation culture with wildtype Psa-V after 12 rounds of subculturing (estimated to $\mathrm{be} \simeq 100$ generations). Figure 3.15 reports the relative ratio of resistant Psa-V (P3) to the wildtype strain, measured at the end of each 24 hour growth period. After each day, the proportion of resistant Psa-V was calculated by dividing the number of streptomycin resistant CFUs by the total number of CFUs from a sample of the co-inoculation culture. The mean ratio of CFUs for resistant to total counts was statistically different to one $(t(10)=4.322, p=0.002$, Mean ratio $=2.63, S D=1.25)$. Monocultures of each strain were maintained in parallel with the co-culture and sampled under the same conditions. Mean CFUs values of monoculture wildtype Psa-V and co-culture samples were not significantly different to one $(t(19)=-0.171, p=0.866)$, which indicates that co-culture sampling was returning values equivalent to the saturated monoculture of wildtype Psa-V. Also, from samples of the resistant Psa-V monoculture there was no evidence for a difference in mean colony count between resistant cultures counted on selective media and colonies counted on non-selective media $(t(18)=-1.325, p=0.202)$, hence a statistically relevant number of resistant Psa- $\mathrm{V}$ was captured on the selective media. This allows the assumption that the resistant CFUs of the co-culture derived from counts on selective media can statistically represent the proportion of streptomycin resistant colonies that make up the total CFUs in the co-inoculation culture.

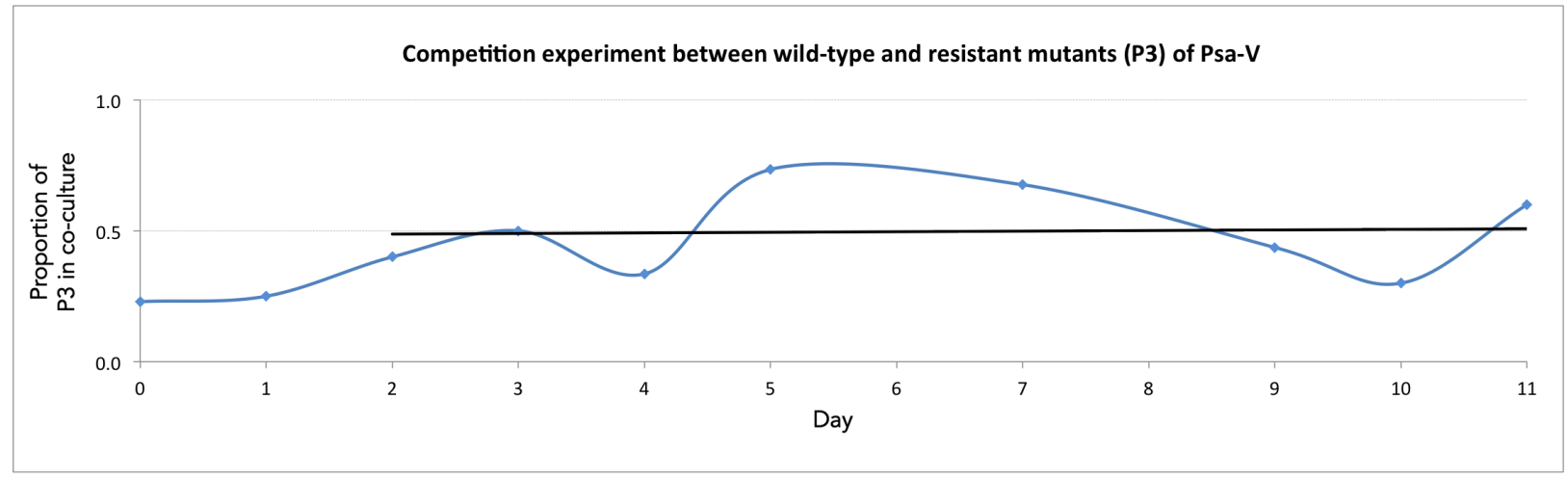

Figure 3.15: Proportion of streptomycin resistant Psa-V CFUs data plotted for each day from a co-culture competition experiment between wildtype and resistant mutants (P3) of Psa-V. Because only one replicate was used in this experiment, due time and material constraints, data point error bars could not be derived. A trend line is imposed from days 2 to 11 shows the average growth of each strain beyond this time is stable and equal. No data was collected for days 6 and 8 .

Ultimately, these data indicate that the streptomycin resistant Psa-V strain did not carry any fitness burden affecting its ability to grow in rich media.

\subsubsection{In planta}

As any fitness effects of the streptomycin resistance mutation may be conditional, it was considered that a colonisation and competition study performed in conditions similar to the natural environment would be likely to give the most relevant results. Obviously an experiment of this nature could not be performed in open kiwifruit orchards lest the 
streptomycin resistant strain escape. Thus, co-inoculation fitness measurements were performed in clonal kiwifruit seedlings growing in controlled and isolated conditions. This required the use of the Beever plant pathogen containment facility (Landcare Research) located in Tamaki, generously made possible by Dr Matt Templeton and overseen by Dr Honour McCann and Dr Stanley Bellgard. Inoculation of the co-culture and monocultures and sampling of the seedlings is detailed in subsection 2.14.2.

Briefly, 16 seedlings were inoculated with a 1:1 co-culture of P3 and Psa-V wildtype (this was produced by mixing equal amounts of freshly resuspended cells of each strain with an O.D. $600 \lambda=0.2$, cells having been harvested from King's B agar plates supplemented with nitrofurantoin). An additional 16 seedlings apiece were inoculated with a mono-culture of either the resistant or wildtype strain i.e. 16 seedlings treated with P3 and 16 seedlings treated with wildtype Psa-V. Inocula of the same density were used for each treatment (O.D. $600=0.2$ ). After inoculation (day 0), four plants for each treatment were immediately sacrificed and $1 \mathrm{~cm}$ (approx) stem samples were taken from the inoculation site of each plant. These stem samples were surface sterilised and mechanically lysed to liberate the bacterial cells within the plant tissue. The lysate was analysed for CFUs per gram of tissue by plating serial dilutions of each sample lysate, and this initial sampling was to give an indication of successful inoculation. This sampling method was repeated with the remaining plants at three and seven days post-inoculation, with six plants per treatment sacrificed at these time points. Aside from the obvious differences in growth conditions, this fitness measurement protocol bears some notable difference to the in vitro assay (section 3.5.1). First, the number of sample points was reduced to three spread over one week, as resources and time were limited for this experiment, due to the requirement for extremely specialised facilities and living plants. Secondly, the CFU measurements were conducted differently from thein vitro experiments to follow optimised methods previously employed by Dr Honour McCann for in planta experimentation and again to reduce resource consumption (Dr Honour McCann, personal communication). Finally, the growth media plates used for CFU measurements required amendment with an additional selective agent (nitrofurantoin) to eliminate endogenous bacteria from the plants. Negative inoculation controls showed that this selective agent was effective in removing non-Psa-V microbes. However, it is possible that this additional media component had a negative effect on the total colony counts.

The first result to be noted from the experiment was the lack of streptomycin resistant Psa-V recovered from the co-inoculation sample on day 0 (Fig. 3.16). In contrast, the monoculture treatment of resistant Psa-V returned comparable levels of CFUs to the monoculture wildtype treatment. Also, both monoculture CFU values were comparable to the CFUs counted for the co-culture treatment on non-selective media for this sample day. Possible reasons for the lack of streptomycin resistant Psa-V measured in the co-inoculation sample may include that on this day the co-culture sample was initially mistakenly plated only on non-selective media, allowing for the growth of both resistant and non-resistant strains. The colonies formed on this non-selective plate were subsequently replica plated onto both selective and non-selective media (i.e \pm streptomycin). However again colonies only grew on the non-selective media, indicating that no viable resistant Psa-V could be 
recovered at this time point. Replica plating was not the standard sampling practice throughout this experiment and may somehow have caused the failure to detect any P3 (resistant) colonies. As at the next time point (day 3) the level of resistant Psa-V recovered from the co-inoculation samples was consistent with the values for the other treatments. So it is reasonable to assume that streptomycin resistant cells were indeed introduced at the time of inoculation and the failure to detect any P3 colonies was a one-off experimental error. Otherwise, this result of an initial zero count followed by a recovery can only be explained by a fitness burden that is only pronounced during the recovery/lag phase of the resistant strain or adaptation to the environment in the plant. For example, this strain was subcultured in the lab many times to induce the resistant phenotype, and in the process it may have become accustomed to rich growth media and optimal lab conditions, causing a transient fitness burden when reintroduced to in vivo conditions [204]. Also, compensatory mutations are known to occur within rpsL mutants that recover fitness after several generations in suboptimal conditions if a resultant fitness burden materialises [205, 206]. Data from later time points however strongly suggest that the plants were heavily inoculated with both streptomycin resistant and wildtype Psa-V from the initial co-culture treatment (Fig. 3.16).

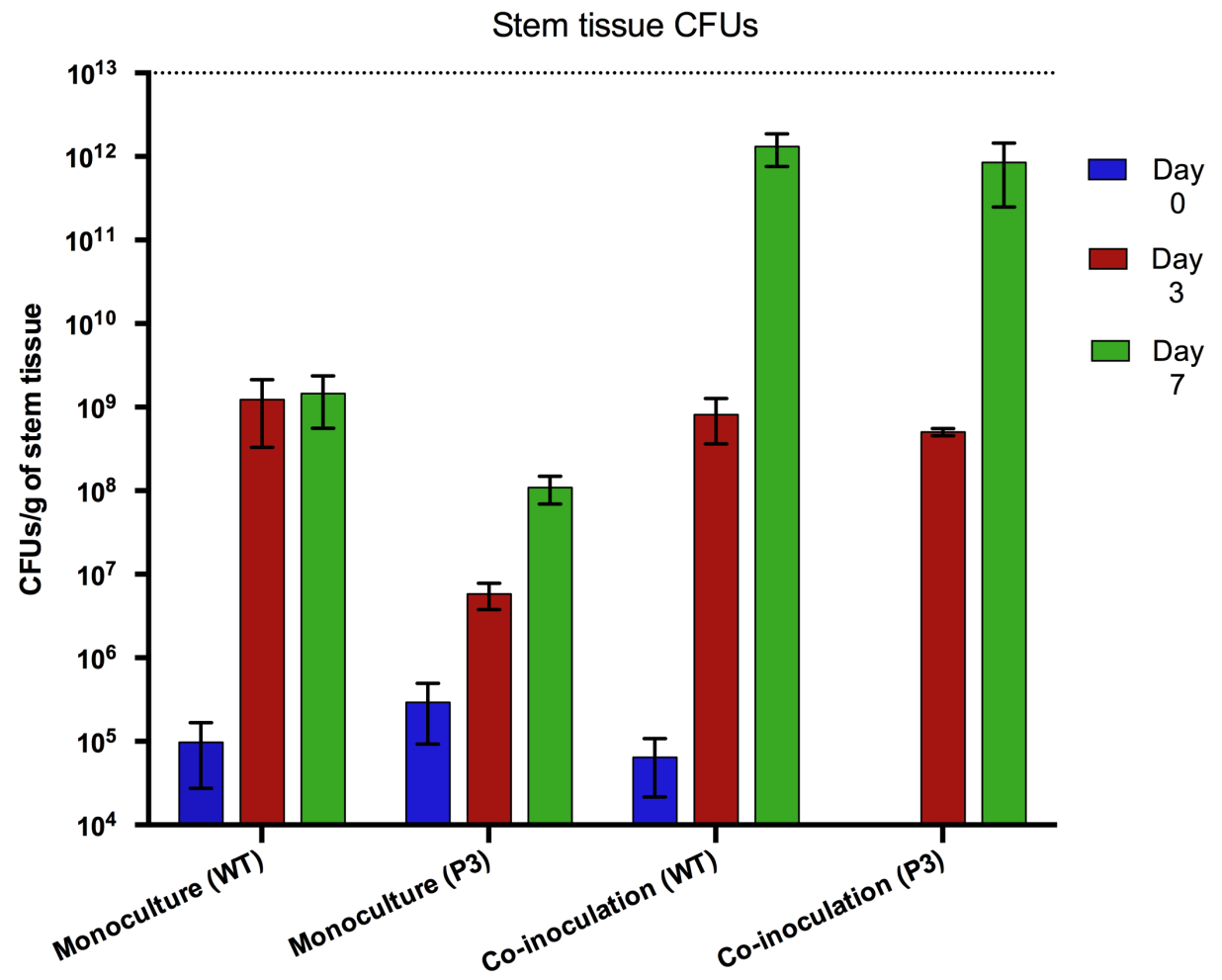

Figure 3.16: In planta co-inoculation results. This figure shows the proportional values of wildtype and P3 CFUs recovered form plants per gram of stem tissue. The co-inoculation wildtype values for each time point are calculated as the average CFUs recovered on non-selective media (i.e. total WT + P3 Psa-V population) minus the average CFUs of resistant Psa- $\mathrm{V}$ from the same samples, recovered on selective media. A bar height of 1:1 would indicate equal proportions of each strain in the co-inoculation treatment. This figure also displays the CFU counts of Psa-V wildtype and Psa-V Strep ${ }^{\mathrm{R}}$ (P3) monocultures. All values are shown on a $\log _{10}$ scale and are the mean of four samples on day 0 and six samples on days 3 and 7 . Error bars are the standard error of the mean. P3 colonies were counted on media containing streptomycin.

CFU counts for the in planta co-inoculation competition assay suggest that streptomycin 
resistant Psa-V maintained a stable population over the seven days. A final population proportion of $39 \%( \pm 28 \%)$ was recorded (calculated as the CFUs of streptomycin resistant cells/total population of resistant and non-resistant cells) at the day 7 time point. Thus, within error, there is no clear fitness advantage in planta for either strain. It is a possibility that the CFU count of streptomycin resistant cells was slightly under-represented or it may be that this strain was slower to establish a population in the plants. In the in vitro experiments the CFU values of a P3 monoculture recovered on streptomycin amended media were on average slightly lower than the values of CFUs of the same monoculture recovered on non-selective media and lower than the CFUs recorded for a monoculture of wildtype. However this difference was not considered significant (section 3.5.1).

Thus, there is no evidence that spontaneously generates streptomycin resistant Psa-V will carry a fitness disadvantage in the field. However, further repeats and perhaps a longer time course are needed to definitively arrive at this conclusion.

\subsection{Discussion}

As part of the strategy to find an appropriate chemical control method, this study generated reproducible data for many compounds on the concentration needed to achieve inhibition of Psa-V in a controlled laboratory environment. Not only did this enable a preliminary evaluation and comparison of novel anti-Psa-V agents, it also provided a baseline measure of activity that might in future have value in monitoring for emergence of resistance to a compound should widespread field use be adopted. As assurance for the suitability of these data for such an application, in a direct comparison of wildtype and streptomycin resistant Psa-V the effective concentrations were found to differ by several orders of magnitude, while measurements of streptomycin's effectiveness on three biological replicates of the wildtype strain were consistent to within $0.5 \mu \mathrm{M}$.

This work has been followed up by ongoing field testing of the products showing significant activity, overseen by Seeka Kiwifruit Ltd. These field tests also include a scoring system to measure phytotoxicity, which could not be gauged by our assay. Nonetheless, the data presented here has not only helped to select viable compounds, but also guided the field trials to appropriate concentrations for initial spray testing (Michael Franks, CEO Seeka Kiwifruit Ltd, personal communications). Fast-tracked product testing has also been conducted by Kiwifruit Vine Health (KVH). KVH's initial in vitro testing do not report $\mathrm{IC}_{50}$ results for the much larger range of products that they tested, but only rank the compounds on a qualitative basis. In sum, very few of these products have shown reasonable activity in the field. Highly active compounds that have gone forward to the KVH Best Practice Spray Programme include the antibiotics KeyStrepto ${ }^{\circledR}$ and Kasumin ${ }^{\circledR}$, as well as a small range of copper-based products. The copper based compounds did not show potent activity in in vitro testing but have made it through field trials, although it is recommended they are applied in conjunction with other proven Psa-V control products (viz. the antibiotic based compounds) and not below the recommend label concentrations, as doing do may lead to suboptimal control and resistance[207]. For the most part, the compounds tested by KVH that overlapped with this study had results consistent with the 
in vitro data reported here [208].

Overall, these findings suggest a narrow range of effective chemical compounds for the control of Psa-V. Moreover the compounds that are available carry limitations (e.g. antibiotic resistance potential or phytotoxicity). As such, the quantification of novel compounds that can inhibit Psa-V is an important area of research.

This study identified the Psa-V inhibitory activity for the agriculturally useful compound niclosamide, provided it is combined with the efflux pump inhibitor PA $\beta N$. As this combination had an inhibitory effect in the same range as the antibiotic compounds it may warrant further study, including field trials to gauge important chemical control properties such as phytotoxicity. If this combination does prove to be a useful field option it may help to preserve the effectiveness of the antibiotics that are already in use.

As niclosamide is not considered a classical antibiotic, but has been used and tested widely in agricultural settings and is also considered safe for humans (e.g. with doses of 2 grams used to treat tape worms [209]), some if the issues typically encountered with registering antibiotic compounds for field application may be avoided. Also important, it is manufactured on a commercial scale and bulk purchasing is available from several chemical suppliers worldwide.

While the same aforementioned characteristics of niclosamide (i.e safety data and generic availability) may not be explicitly true for PA $\beta \mathrm{N}$, it is considered a gold standard efflux pump inhibitor, hence its use in this study, and as such there are many published studies that have used PAßN as an antimicrobial adjuvant although it has is not yet been used therapeutically [210]. Several other efflux pump inhibitors have been discovered and developed $[211,212]$ which may expand the available options for the application of promoting niclosamide sensitivity in Psa-V.

In this research, the established antibiotics were generally found to be the most effective compounds including several options that are either not in clinical use or are already used in other agricultural settings and were hence plausible for field trials in kiwifruit. While oxytetracycline has been used in field trials in Japan and was found to be highly effective against Psa-V in this study, the fact that this antibiotic still has clinical applications is likely to prove off-putting in New Zealand relative to the two most plausible antibiotic candidates to emerge from this study (i.e. kasugamycin and streptomycin). Importantly, both these antibiotics have differing ribosomal sites of action, suggesting that in combination they may prove effective in controlling Psa- $\mathrm{V}$ while reducing the incidence of antibiotic resistance.

Further investigations were carried out even as the antibiotics streptomycin and kasugamycin were being adopted for field use. In a series of experiments, spontaneous streptomycin resistance was repeatedly forced in Psa-V. However, the same approach did not result in the generation of kasugamycin resistance. As high-level resistance to streptomycin only required one SNP, which can be spontaneously induced simply by exposing wildtype Psa-V to the antibiotic, it can be assumed that this will readily occur in the field where streptomycin is being used. A research scientist from Verified Lab Services (Te Puke), responsible for overseeing a majority of Psa-V detection in the Bay of Plenty and holding a vast collection of field isolate from this region, has been contacted to request isolates from orchards where streptomycin is being been used. In the future it is planned to 
test the streptomycin sensitivity of these field isolates, from before and after the introduction of the antibiotic, to look for resistance development. These isolates did not arrive within the timeframe of this study, although it has been suggested streptomycin resistance is yet to be reported in the field (Shalinee Dowlut, personal communication).

The lab-induced streptomycin resistant mutants were used in co-culture experiments, both in vitro and in planta, with the aim of quantifying any relationship between fitness levels and resistance. Although preliminary at this stage, the results of these experiments may one day help guide field practice of antibiotic use as a control measure for Psa-V. Many studies have reported that the trajectory of resistance and its possible reversal is directly related to the cost that the resistance mechanism imposes on the fitness of the bacteria [196]. Therefore, for each of the three scenarios suggested in section 3.5, a different application strategy may necessary to maintain maximum efficacy of the antibiotics in use. To draw definite conclusions from the data supplied by this study alone would be short-sighted, as the collection of data from sufficient replicates and time points was not feasible given the resource and time constraints confronted in this study. However, the questions and initial data produced has already garnered the interest of other parties concerned with Psa-V control (Dr David Ackerley, personal communication).

With further study, relevant recommendations on antibiotic practices could be made to maintain, or at least prolong, the benefits of streptomycin use. In the first instance, should a significant fitness cost be found to occur in resistant strains, to reduce or abstain from streptomycin use may diminish or ablate the population of the resistant strain as it will suffer from competition by the fitter wildtype strain. This prospect encourages testing for a irrecoverable fitness cost that could result in reversing the prevalence of resistance by suspending the use of streptomycin and substituting or amending it with another antibiotic. However, my results to date suggest this is not the case. In the second and third scenarios, where resistance mutations ultimately lead to no apparent fitness cost, the most sensible option may be to apply an additional antibiotic to impede resistance from arising. Kasugamycin is the obvious candidate for this, as it is already approved for use and it appears to have a much lower propensity than streptomycin in provoking resistance. However, testing this theory would require additional experimentation in conjunction with completing data sets on streptomycin fitness costs and examining for cross-resistance of additional antibiotics. 


\title{
Chapter 4
}

\author{
Experimental Results - Psa-V Knockouts
}

\subsection{Introduction}

The principal goal of the research described in this chapter was to create targeted gene knockout strains of Psa-V, by cleanly removing genes from its chromosome (leaving an otherwise isogenic background), to produce strains which lacked one or more essential virulence determinants. The intended purpose of the knockout mutations were two-fold: first, to elucidate the function of possible virulence factors and measure their contribution to the disease phenotype seen of Psa-V; and, secondly to develop an isogenic non-virulent strain of Psa-V that might prove effective as an antagonist against wild type Psa-V and therefore be useful as a bio-control agent. To achieve this it was first necessary to conduct a detailed background study around the feasibility of this strategy; and hence this chapter includes a comprehensive literature review and strategy development section prior to the presentation of experimental results.

A significant proportion of the targets were selected at the onset of the project and these selections were based on literature findings of known virulence factors from related plant pathogens. Although the selection of knockout gene candidates was not static throughout the duration of this study, new nominees were added to (or removed from) the candidate list as parallel genome sequencing efforts from out collaborators revealed additional targets of interest. These circumstances resulted in the initial method for the selection of targets being limited to finding homologues of known virulence genes, informed by literature searching, in early fragmented drafts of the Psa-V genome. Once a high quality and comprehensibly annotated whole genome was available several new knockout targets were added. This was partially because of third party interest in gene function, and thus the germane knockout strains, was now being expressed but also because interesting new Psa-V specific targets that could not found prior to the assembly of a complete Psa-V genome sequence were now evident.

\subsubsection{Biocontrol agent (BCA) modes of action}

Across the field of BCAs, several concepts regarding phytopathogen antagonism have been explored and are worthy of general consideration here for insight to the possibilities that may be applied to Psa-V. The common goal with BCAs is to have an introducible non-pathogenic strain that can competitively colonise plant tissues and has an ability to 
reduce the population of pathogenic bacteria and thus the incidence of disease [214]. The methods of pathogen inhibition by BCAs is through either antibiosis, direct competition for space and essential nutrients or by inciting host defences. [215, 216].

The antibiosis concept was noted when differential results in plant growth and disease susceptibility was seen from site to site by horticulturists as early as the 1920s [217]. It was soon recognised that a microbial agent in the soil was responsible for this effect [218]. The phenomenon can cause a reduction of the pathogen population, e.g due to antimicrobial compounds, diffusible or volatile secondary metabolites, produced by a BCA [46]. Many Pseudomonas spp. have been implicated and tested as BCAs in the rhizosphere with the production, secretion and effects of the antibiotics produced well characterized [219]. The fluorescent

Definitions:
ANTIBIOSIS: A condition in which
one or several metabolites that are
excreted by an organism have a
harmful effect on other organisms.
PHYLLOSPHERE: The aerial habitat
of plants colonized by microbes [213].
RHIZOSPHERE: A nutrient rich zone
near (that is, a few millimetres from)
the roots, where microbial growth is
stimulated by root exudates (the rhi-
zosphere effect).
biocontrol and plant growth-promoting rhizobacteria (PGPR) strain P. fluorescens CHAO, isolated from a natural disease-suppressive soil in Switzerland, can produce the antibiotics 2,4-diacetylphloroglucinol, pyoluteorin, and pyrrolnitrin. These compounds are evidently the primary mechanism of bio-control by fluorescent pseudomonads [220]. Research into this method of antagonism, i.e. the excretion of secondary metabolites, is restricted predominantly to rhizosphere colonizing BCAs [217]. Interestingly, some strains of PGPR which had been mutated to not produce the antibiotic thought to be responsible for antagonism still maintained a similar degree of antagonism to their wild type counterparts, whereas other studies have shown that the production of certain metabolites was essential to the antagonistic effects [221, 222]. I raise this notion to illustrate the complexity that can occur with interspecific competition, even within the elementary concept of antibiosis, and to demonstrate that simple competition for resources may be sufficient for effective biocontrol.

Overlapping with the antibiosis concept but progressing up to the phyllosphere, and also transitioning to the premise of direct competition, is antagonism by the production of siderophores. Siderophores are iron-binding ligands secreted by microorganisms to allow sequestering of iron, an essential micro-nutrient, from environments of low iron availability. The fluorescent pseudomonads owe their characteristic yellow/green fluorescences to pyoverdine, a potent siderophore produced and excreted by these strains. This particular siderophore has very high affinity for $\mathrm{Fe}^{3+}$ ions (its stability constant for $\mathrm{Fe}^{3+}$ was measured to be $1.89 \times 10^{24}$ at $\mathrm{pH} 7$, determined by Meyer et. al. [223]). As situations of low iron availability are apparent in some soil conditions and on leaf surfaces [213] the ability to produce siderophores becomes a selective advantage for the producer in these environments and plays an important role in the differential survival of resident microorganisms. Furthermore, siderophore production by one microorganism to exclude other microorganisms from the surrounding micro-environment, by diminishing already low concentrations of iron, has been suggested as an effective biocontrol trait. Under certain conditions, some siderophores have been shown to have bacteriostatic and/or fungistatic 
effects [218]. The situation of enhanced epiphytic fitness and exclusion of pathogens by siderophore production, conferring bio-control properties to a strain, is usually seen between unrelated species that inhabit similar niches. For example, the siderophore producing P. putida strain B10 inhibits the eukaryote Gaeumannomyces graminis var. tritici, a fungal wilt/take-all disease. In iron amended soil or media, which represses siderophore production, this inhibition is lost. Evaluation of BCAs and siderophore production highlights that some known PGPR with pyoverdine negative mutations have a reduced ability to protect the host plants compared to the wild type PGPR, although this is not always the case. Also apparent, most strains of fluorescent pyoverdine producing pseudomonas are not useful as BCAs. Therefore, in some instances pyoverdine mediated iron deprivation contributes to disease suppression but alone is not sufficient to explain the biocontrol activity of fluorescent pseudomonads [46, 218, 224].

Between directly related species or strains, i.e. where the same siderophore(s) are used by the antagonistic strain and the pathogen, production of the iron-chelating agents maybe important to the fitness of the BCA but is not the method by which it antagonizes. In one of the more robust and repeatable BCA systems characterized to date, Wensing et al., (2010) [225], shows that this is the case with the antagonism of $P$. syringae pv. glycinea by P.s pv. syringae $22 \mathrm{~d} / 93$ on soy-bean plants. P.s pv. glycinea is the causal agent of bacterial blight of soy-bean and the results of field studies, published by Völksch and May (2001), demonstrated that the strain P.s pv. syringae $22 \mathrm{~d} / 93$ could be used as a BCA against the pathogen. Siderophore deficient P.s pv. syringae 22d/93 mutants retained the same biocontrol properties as the parental strain and were able to reduce pathogen population size and disease scores. This suggests siderophore production and uptake by $P$. syringae pv. syringae $22 \mathrm{~d} / 93$ is not important in the direct biocontrol activity against the related pathogen species. [226, 227].

Direct competition for available nutrients or niche micro-environments may contribute to the effectiveness of BCAs that are closely related to the pathogen which they are being utilised to control. There are several different strategies that can produce this direct competitive pressure. One strategies is to take hold of an optimal growth niche, divide rapidly and protect the resources by expanding the population to limiting densities (this is known in population dynamics as scramble competition [228]). Another strategy is to displace bacteria that are already established by some means of clearing out the existing residents, usually by the production of antimicrobials or molecules that disperse other populations without actually killing the cells, and then recolonising the niche [228, 229]. The choice of strategy becomes very important when biocontrol is to take place at the level of the phyllosphere, especially scramble competition where closely related strains are concerned. There is evidence that scramble like behaviour occurs when Psa-V colonises leaf surfaces. Microscopic examination clearly shows a rapid population expansion of Psa-V at, and only at, the stomata [230] (Fig. 4.1). Once this is niche is occupied successful new colonisation events by any other bacteria is unlikely, because the reproductive success of an immigrating cell is inversely proportionate to the density of the established bacterial population [231]. 


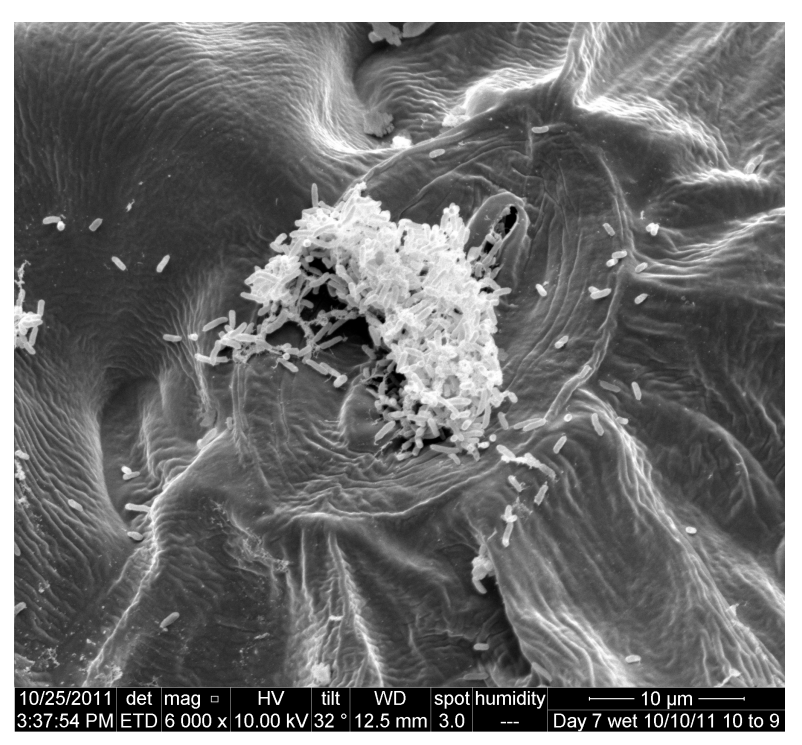

Figure 4.1: Psa-V colonisation of stomata. This scanning electron micrograph maybe depicting a hypothesised preferential colonisation and population expansion of Psa- $\mathrm{V}$ at the site of stomata on kiwifruit leaves, the static image may also however be interpreted as showing Psa-V extruding from the stomata. Image credit: Paul Sutherland, Plant \& Food Research. Used with permission.
The population dynamics of bacteria that inhabit the phyllosphere is largely determined by the ability of strains to differentially tolerate the low levels of nutrients and the harsh regularly fluxing conditions found on the leaf surface. [232, 233]. The fate of bacterial immigrants colonising a leaf surface is dependant on where on the leaf they land, the level of inoculation and how heavily colonised the sites of deposit already are. This is due to the variation in local carrying capacities at different sites across the leaf surface. The local carrying capacity of distinct sites on the leaf is linked to the nutrient availability at each site.

Laboratory models, using stable intracellular green fluorescent protein (GFP) dilutions to measure individual cell division, suggest that there are only a few sites on each leaf that will allow a relatively high level of reproductive success [231]. The experiments, using Erwinia herbicola loaded with GFP, showed most bacteria did not divide after an inoculation event. In these experiments the cells are the perfect isogenic competitors for each other and will utilise the exact same complement of resources giving a niche overlap index (NOI) of 1. This index can be estimated for pairs of bacterial species by examining the differences in in vitro carbon source utilisation profiles and gives an indication of niche similarity. Between pairs of species, this index was inversely proportional to the co-existence of one species with respect to the other, when measured by replacement series experiments [234]. The replacement series is a substitutive experimental design where two species are inoculated as a mixture at varying levels but always to the same total concentration. The proportion of each species it then determined after community equilibrium is reached. In species pairs with a low NOI, each species will utilise different carbon sources and when co-existing on the resource limited environment of the phyllosphere each species population will grow to a level closer to that seen when only there is one species is present. When the NOI is high, seen between isogenic or near isogenic pairs, the replacement of one species (or strain) by another will result in a low level of co-existence, which is assumed to be a consequence of the direct competition for one or more resources [235]. Thus, antagonism due to the competition of one strain with another for resources will increase proportionally with the overlap of their ecological niches and it is expected that this niche overlap will be proportional to the genetic relatedness between the pairs.

An application of direct competition in field trials is seen in studies of the near isogenic strains of the ice nucleation-active $P$. syringae pv. syringae $\left(\mathrm{Ice}^{+}\right)$and ice nucleating-deficient mutants $\left(\right.$ Ice $^{-}$). These trials were set up when it was discovered that the presence of certain bacteria, including $P$. syringe, were responsible for the nucleation of 
ice, causing frost damage to plants at appreciably higher temperatures than was observed when the bacteria were absent [236]. The cause of ice nucleation was subsequently discovered to be a glycosyl phosphatidylinositol-anchored outer membrane protein found in some gram-negative bacteria [237]. It was reasoned that if the population of Ice $^{+}$bacteria carrying the ice-nucleating protein (Inp) could be replaced by an Ice ${ }^{-}$strain the plants could be spared from frost damage. It was proposed that when an $\mathrm{Ice}^{-}$strain was sprayed onto plants at very high concentrations, the naturally occurring $\mathrm{Ice}^{+}$bacteria would not be able to compete. The first experiments carried out using Ice ${ }^{-}$mutants of the parental strain showed that pre-inoculation with an Ice ${ }^{-}$mutant would reduce frost damage severity as well as the potential population size of Ice $^{+}$strains [55]. This study discusses competitive exclusion (i.e. reducing subsequent colonisation) as the means of protection by the near isogenic strain from frost damage, rather than competitive displacement (i.e one strain causes a local extinction of another). The results of these studies were significant enough to warrant further research into the biocontrol of frost protection. Although generally regarded as safe in this instance, the use of genetically altered bacteria to control ice nucleation was met with considerable resistance. Researchers subsequently focused on naturally occurring strains and isolated P. fluorescens A506, this strain had similar biocontrol activity towards Ice ${ }^{+}$bacteria and was not genetically altered. This strain also had protective qualities against Erwinia amylovora [48] and due to the commercial application A506 was marketed as Blightban A506 ${ }^{\circledR}$ to protect against fire blight on pear and apple trees [238]. An isolate of non ice-nucleating Erwinia herbicola has also been patented for protection against frost damage [239].

Expanding further on the concept of competition for resources as a means of antagonism between a BCA and a pathogen, studies have investigated the use of unrelated strains as BCAs which can degrade a particular essential nutritional requirement of the pathogen. Of interest is the control of E. amylovora by P. rhizosphaerae JAN. The ability of JAN to control th population size of E. amylovora on the shoots and blossoms of apple, and on pear slices was measured directly against that of the commercial A506 strain of P. fluorescens. In this head to head comparison, JAN was better able to control the pathogen than A506 in green house conditions. This superior antagonism was accredited to JAN metabolising nicotinamide and nicotinic acid (NAM/NAc) [240], as at least one of either of these is required as a essential growth factor for the survival of E. amylovora [241]. Non NAM/NAc degrading mutant strains of JAN did not have the ability to protect against the pathogen. Previous knowledge of the NAM/NAc requirements of E. amylovora allowed the authors to do high throughput screening of naturally occurring strains, isolated from the same environment that the pathogen would be encountered, for possible biocontrol activity. This approach had several advantages. Media based pre-screening for NAM/NAc metabolism remarkably reduced the number of isolates required to go through resource intensive BCA assays that require living host tissue. Also, as this system does not rely on the production of any antibiotic factors, any off target activity, towards say other beneficial bacteria, is largely reduced. Finally, by using this method the resulting strains will be conditioned to survive in the appropriate environmental niche.

One more proposed mechanism of pathogen antagonism will be mentioned before moving 
on. This is the mechanism where by the plant's natural defences against bacteria (as well as fungi and insects) are unregulated by a BCA, inducing an environment inhospitable to any invading pathogen. The signalling pathways involved are dealt with in section 1.5 of this chapter 1, but an overview will be offered here along with examples of field application.

Plants have evolved necessary natural defences against bacterial pathogens. This defence lies essentially dormant partly in order to conserve resources but also because the extreme end of the defence spectrum is the induction of localised plant cell death. A broad-spectrum defensive state is triggered when a plant recognises the appropriate stimuli [219], from either a pathogen or other external exogenous elicitors [242]. In response to the pathogen or elicitor, distal regions of the plant are also stimulated, by systemic signals, into a defensive state effective against a wide range of pathogens, including those taxonomically independent to the entity initially provoking the reaction. This is known as systemic acquired resistance (SAR) and signalling is mediated, in part at least, by salicylic acid (SA). The key to a successful defensive campaign is a timely recognition of the pathogen and an upregulation of the genes required to mount the response. To this end, BCAs or chemical elicitors can be used to "prime" a host's defence well before the invasion of a pathogen. Field trials to evaluate the protection of grapevine leaves against a fungal pathogen Botrytis cinerea by several different bacteria capable of inducing resistance reactions were carried out in two vineyard orchards located in Champagne (France) [243]. The experiments involved seven grapevine associated bacteria with previously reported in vitro biocontrol activity, it used three different methods of application and was conducted from 2002 to 2005 over two sites. Results were generally positive and high levels of statistically significant protection were shown in many sets of trials. For example, in one set of trials, where leaves were sprayed with $P$. fluorescens strain PTA-CT2 and then inoculated with highly virulent B. cinerea, a 39-43\% reduction of necrotic legions compared to unprotected control leaves was observed. A five fold increase in chitinase activity and a four fold increase in $\beta-1,3$-glucanase activity was recorded in the leaves of plants that had the PTA-CT2 strain applied by soil drenching. Chitinase and $\beta-1,3$-glucanase activity $[244,245]$ were used in this study as a measure of induced defence as they are activities of known pathogenesis-related proteins usually associated with SAR.

\subsection{Virulence mechanisms as targets for control}

In light of the summarised information above that confirms bacterial biocontrol of pathogens is achievable by several modes of action, including the use of gene knockouts strain as antagonists, genes or functional types of genes were sought as targets for knockout experiments with the objective of creating a strain of Psa-V with biocontrol properties. The function(s) interpreted as qualifying candidate genes to participate in this goal are summarised below in sections 4.2.1 to 4.2.5. The viability of Psa-V knockouts of these selected targets was assumed based on the existence of similar or homologous knockouts in related strains. Also included here are the target members that were selected with the added interest of elucidating gene function or virulence contribution, by definition all of the targets specific to Psa-V fall in to this category. These two initiative were considered to be 
complementary, for example an effector protein knockout may give insight into the virulence contribution of the effector as well as yield a strain with decreased virulence and or biocontrol properties.

\subsubsection{GacA / GacS}

The GacA and GacS proteins make up a two-component environmentally responsive transcriptional regulation system. This system has been found in many Gram-negative plant associated bacteria, especially the pseudomonads [246], and it has been independently shown to control bacterial genes that produce extracellular products involved in environmental manipulation and virulence [247]. The system is activated when the membrane bound sensor kinase GacS becomes autophosphorylated in response to an unknown extracellular signal. This unknown signal may be synthesised by the host or, in a quorum dependent manner, by the bacteria themselves linking the system to bacterial population density. This activating signal is then passed on to the global transcriptional regulator GacA by phosphorelay resulting in positive transcriptional regulation of target genes $[248,249]$. The genes under the control of GacA are involved in synthesis and secretion of extracellular enzymes, secondary metabolites and quorum molecules. This makes the system important in regulating traits of pathogenicity, ecological fitness and stress tolerance, and relates these responsive traits to the environment and cell density. The GacS/GacA system constitutes a switch between primary and secondary metabolism and GacS/GacA null mutants of $P$. syringae have a markedly reduced virulence as they are unable to switch to an invasive state [16].

\subsubsection{Quorum sensing}

The local concentration of quorum sensing (QS) molecules, small diffusible molecules produced and excreted by colonising bacteria, can regulate the motility and virulence of a bacterial population. In many strains of phytopathogenic pseudomonads, $N$-acyl homoserine lactones (AHLs) are the most common excreted QS molecules which are produced by synthase proteins in the LuxI family[250]. The concentration of QS molecules in the local environment is dependant on the bacterial population density. Upon detecting a threshold concentration of an excreted QS molecule with a cognate transcriptional regulator, bacterial cells are triggered to switch metabolic profiles and change behaviour from non-virulent to invasive [251]. For example, the size, location and density of an epiphytic population of $P$. syringae pv. syringae, the laboratory model organism and bean pathogen [14], is directly proportional to the occurrence of host disease. This observation implies that to support survival during the plant's initial line of defence, P. syringae pv. syringae uses QS to build up a large population density on certain parts of the leaf before initiating virulence and invading, essentially ambushing the host [252]. This organised attack strategy synchronises the bacterial population and is necessary for invasive success because individual cells would not produce a concentration of virulence factors capable of overwhelming the plant [253]. As such, the anticipated QS system genes in Psa-V was proposed as targets for knock-out, early in the course of the study before full genetic data 
was available, to produce a viable strain lacking the ability to stage a noxious invasion. As Psa-V genome data became available, homologs of common QS molecule synthase genes and enzyme sequences from related species (such as AHL-synthase (ahlI) from P. syringae pv.syringae) were queried against Psa-V data. No significant hits for an AHL synthase like gene were found in this study. At this time the Psa-V the extracellular quorum signalling molecule and the genes that might produce such a molecule remains unknown and it has been reported that Psa-V does not any AHL like molecules [254]. To complete the QS system, the excreted QS AHL molecules are detected by intracellular transcriptional regulator proteins of the LuxR-family, often found encoded in tandem on the genome, to give a QS LuxI/LuxR pair [250]. Although Psa-V does not appear to contain any luxI genes, it does however contain three genes encoding for LuxR homologs [254]. It is not uncommon for phytopathogenic bacteria to posses an incomplete QS system consisting only of proteins of the LuxR family. These unpaired LuxR proteins, termed "solos", are thought to be important in detecting QS molecules from other bacteria in mixed populations or, in special cases, plant derived molecules [255]. The study which identified the LuxR solos in Psa-V (Patel et al. 2014) went on to produce knock out strains in Psa-V for each of these genes and showed markedly reduced survival and multiplication after 3 and 7 days when inoculated on the leaves of $A$. deliciosa and A. chinosa, compared to wild type Psa-V. This suggests that these QS related genes play an important in the virulence level of Psa-V although effects of these knock out strains as a biocontrol agent were not reported.

\subsubsection{Type III secretion system (T3SS)}

P. syringae, the T3SS is encoded by the hrp (hypersensitivity response and pathogenicity)/hrc (hypersensitivity response and conserved) gene cluster which produces the 25 different proteins making up this structure [114]. This cluster was obtained by $P$. syringae preceding the differentiation of the species into its many pathovars. The T3SS features a hollow needle like filamentous macromolecular surface appendage that is of sufficient length (in the micrometer range) to provide a continuous channel for effector proteins to pass through both the bacterial and plant cell walls and membranes [256]. Expression of the T3SS is necessary for bacterial pathogenicity and for inducing the hypersensitivity response, but not for PAMP-triggered immunity, suggesting it may not necessarily be detected by pattern recognition receptors on plant cell membranes.

Assembled into a hypodermic needle like structure, the T3SS allows unfolded effectors to be transported across the two membranes of the Gram-negative P. syringae cell wall and the membrane of plant cell. It is not until the effectors are inside the plant cell that they perform the interactions that mediate immune suppression or elicit the HR, depending on the host defence adaptations. In P. syringae pv. tomato DC3000 the hrp/hrc gene cluster, which contains all the genes to produce the T3SS, is preceded by the conserved effector locus (CEL) which encodes effector proteins important for parasitic fitness and is followed by the exchangeable effector locus (EEL) in a tripartite mosaic structure. The CEL component is generally conserved throughout $P$. syringae pathovars, while the EEL sequence section has a lower $\mathrm{G}+\mathrm{C}$ content, contains many exchangeable elements and 
possesses genes encoding some of the effectors that vary between pathovars and strains. Together this tripartite mosaic structure is known as the Hrp pathogenicity island. Thus, it appears that Hrp-mediated pathogenicity of $P$. syringae is collectively dependent on one set of genes that are universal among divergent pathovars and on another set that can vary even between strain of the same pathovar [114]. In Psa-V, the hrp/hrc gene cluster is present with some proximal effector genes although most of the effector genes are distally distributed, alone or in clusters, throughout the genome. As the T3SS is required for virulence, but may not have such marked effects on epiphytic fitness, key elements of the Hrp pathogenicity island may provide suitable targets to disrupt the overall pathogenicity.

\subsubsection{Psa-V effectors}

Psa-V contains putative effector genes that are conserved amongst the Japanese and pre-2008 Italian Psa isolates and Psa-V. These genes were identified by comparison to other $P$. syringae strains. It was also found that Psa-V did not contain hop $A B$ and hopAF which are otherwise considered conserved effector genes in $P$. syringae and present in other strains of Psa. Psa-V does however contain the effector genes hopA1, hopAA1-2, hopH1 and hopZ5 that are absent in other strains of Psa [34], although hopH1 is disrupted and most likely non-functional in Psa-V. hopZ5 is a particularly interesting knockout target as it has not been found in any other species of bacteria before. In terms of plant immunity, the loss of one or more effectors may be an advantage to the pathogen as the plant can no longer recognise these particular effectors to trigger ETI. The loss of effectors may be supplemented by the gain of new effectors, through horizontal gene transfer, that replace the function of the lost effector; thereby increasing bacterial virulence until the plant can evolve to recognise the novel effector combinations. As Psa-V has evolved against the static monoculture of cultivated kiwifruit, a highly virulent pathogen has developed towards a population of hosts that lacks the genetic diversity to evolve resistance without human intervention. As Psa-V possesses a unique effector complement compared to other Psa strains, targeting these to reduce virulence may be a viable option. Irrespective, knockout strains may prove useful as a research tool to study the unique host pathogen interactions.

\subsubsection{NRPS gene cluster}

It is yet unknown how or if the gene cluster overviewed in section 1.6.7 contributes to the phenotype of the pathogen. But, as it may generate a toxin and it is unique to Psa-V it suggests itself as a promising knockout target. Moreover, other researchers are interested in the elucidation of the products of these genes (Dr Matt Templeton, personal communication). Knocking out the cluster may help in collaborative efforts to elucidate any role that it plays in pathogenicity, and a knockout strain may serve as a useful background for metabolic profiling to identify the product of this gene cluster (e.g. by using 2D NMR approaches to create metabolic maps of the wild type and mutant strains and then subtracting the latter from the former to identify key metabolite peaks that remain). 


\subsubsection{Knock out target summary}

As noted, one of the primary goals of this work was to develop an avirulent but otherwise isogenic strain of Psa that is able to colonise the leaf and compete for limited environmental resources. This could potentially antagonize the pathogen population density on the leaf to below the threshold needed to cause invasive disease. As the same growth conditions are favoured, closely related strains will cause the most intense impact when in competition. Knockouts of different targets in Psa-V could produce very closely-related strains that may still have the ability to colonise the epiphytic niche effectively and apply a competitive force against fully pathogenic wild-type strains. The genes candidates for this project are presented below (Table 4.1). As the final outcome of this aspect of the study would be to use the engineered strains in the field, consideration has been placed upon the method of genetic manipulation used to produce novel strains. The proposed method of gene knockout was by targeted allele exchange where the target gene is swapped for an in-frame truncated version with no additional genetic material introduced. This targeted approach which leaves no foreign genetic material in the resulting bacterium may circumvent some of the regulatory restrains on the release of genetically modified organism release in the field as genetically modified organisms in New Zealand have always been defined by legislation by means of their creation. The Environmental Protection Authority has been contacted for clarification of this point but so far no official response relating specifically to our proposed knockout methodology has been received. 
Table 4.1: Candidate genes as knockout targets

\begin{tabular}{|c|c|c|c|}
\hline Gene & Function & Reference & (\% Amino acid identity) \\
\hline $\operatorname{alg} U$ & Virulence factor. Regulatory gene of biofilm and attachment. & {$[257,258]$} & Match $(99.5 \%)$ \\
\hline$g a c A$ & $\begin{array}{l}\text { Regulates pathogenicity factors, quorum sensing, and toxins. } \\
\text { Also genes involved in motility, biofilm formation, extracellular } \\
\text { polysaccharides. }\end{array}$ & {$[251,258]$} & Match $(99.5 \%)$ \\
\hline$a h l R$ & $\begin{array}{l}\text { Regulates acyl homoserine lactone (AHL) production by } \\
\text { positive feedback. Needed for expression of AHL-synthase. }\end{array}$ & {$[251,259]$} & No match \\
\hline$g a c S$ & Related to $g a c A$, shown to influence motility. & {$[16,259]$} & Match $(94.7 \%)$ \\
\hline hrpK & $\begin{array}{l}\text { Effector translocation. DC3000 mutants lacking hrpK have } \\
\text { reduced disease symptoms and multiplication in planta. }\end{array}$ & {$[258,260]$} & Match $(97.4 \%)$ \\
\hline$h r p L$ & $\begin{array}{l}\text { Sigma factor. Transcriptional factor controlling expression of } \\
\text { most } h r p, h r c, \text { and avr genes. }\end{array}$ & {$[261,262]$} & Match $(99.5 \%)$ \\
\hline $\operatorname{hrp} A$ & T3SS pili subunit. & {$[89,263]$} & Match $(98.2 \%)$ \\
\hline$h r c V$ & T3SS secretion protein. & {$[259,264]$} & Match $(92.7 \%)$ \\
\hline hopH1 & Effector gene. & {$[20]$} & Match $(100 \%)$ \\
\hline hopZ5 & Effector gene. & {$[20]$} & Psa-V specific \\
\hline hopA1 & Effector gene. & {$[25]$} & Frame shifts \\
\hline NRPS & $\begin{array}{l}\text { Putative toxin synthesis cluster. Two amino acid adenylation } \\
\text { domain targets in cluster (N1 and N2). }\end{array}$ & {$[20]$} & Psa-V specific \\
\hline$m t a P$ & $\begin{array}{l}5^{\prime} \text {-methylthioadenosine phosphorylase. Quorum sensing } \\
\text { inhibitor target. }\end{array}$ & 1 & Match $(97.2 \%)$ \\
\hline 3170 & $\begin{array}{l}\text { N-ethylammeline chlorohydrolase - Atz/Trz family. Quorum } \\
\text { sensing inhibitor target. }\end{array}$ & 1 & Match $(98.3 \%)$ \\
\hline
\end{tabular}

${ }^{1}$ Personal communication, Dr Richard Furneaux. 


\subsection{Experimental results}

The results from each step of the knockout approach used in this project are detailed below.

\subsubsection{Gene truncation}

The gene knockout approach first required the synthesis of truncated target genes, which were made using the splicing by overlap extension PCR protocol described in section 2.1. For each target gene two primer pairs (four primers total per target) were designed, these amplified two fragments which were subsequently spliced by overlap PCR to produce the truncated gene. The overlap reaction was designed to produced a $2 \mathrm{~kb}$ fragment that replaced the full target gene with a sequence capturing only the first and last three codons of the target gene. As an example, a representation of the primer design process and the expected PCR products produced used for truncating the target genehopH1 are shown below (Figs. 4.2, $4.3 \& 4.4$ ).

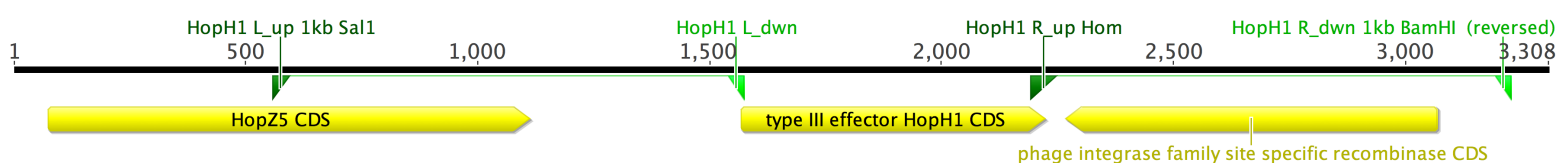

Figure 4.2: Overlap primer design overview example: Relevant region $(\sim 3.3 \mathrm{~kb})$ of the Psa-V chromosomal sequence (solid black line) showing the template regions and primer binding positions of the primer sets used in synthesising the truncated hoph1 fragment. The figure shows the position of the two primer sets required for the two reactions which resulted in left and right hand fragments that were later spliced in a single overlap extension reaction. The positions of the gene coding sequences are represented here as yellow bars with the target gene, hopH1, being in the centre and flanked by the primer sets. Dark green triangles - forward primers, bright green triangles - reverse primers. The thin green line between the primers indicated the region of sequence to be amplified for the relevant primer set.

A 25 bp homology region was designed into the primer sets so that the two resulting PCR products would have $25 \mathrm{bp}$ of overlapping sequence to allow for the directional splicing by overlap extension of these products. This homologous sequence was incorporated into the primers, that had the suffix R_up_Hom, as a 5‘ overhang.

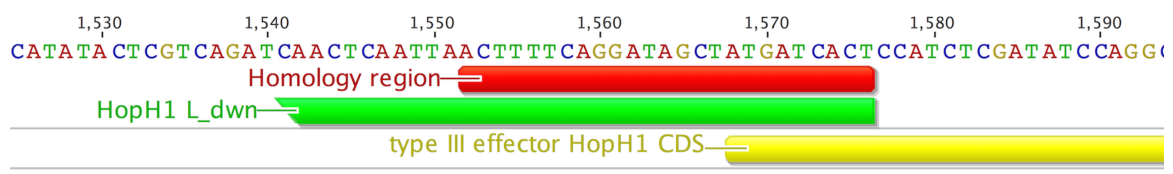

Figure 4.3: Detail of the position of the example primer HopH1_L_dwn relative to the start of the hoph1 coding sequence with the sequence area that informs the homologous region to be incorporated into primer HopH1_R_up_Hom above the solid red bar.

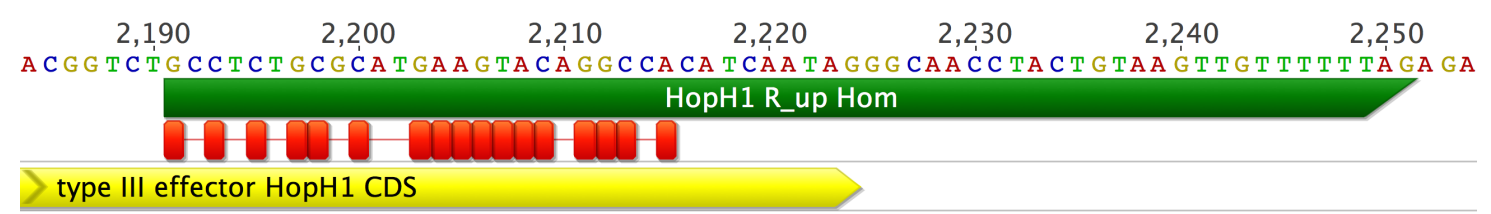

Figure 4.4: Detail of the position of the example primer HopH1_R_up_Hom. There are 5‘ mismatches in this primer caused by the incorporation of the homologous region which was required for the next overlap PCR step. The positions where the primer does not match the shown template because of this $5^{6}$ incorporation are indicated by red marks. 
Using the information of the primer and target sequences, the expected PCR product sequences were predicted in silico and then concatenated together in the correct orientation to give the final expected overlapped product containing the truncated target gene.

All knockout vector constructs were verified by sequencing the insert region of the plasmid using plasmid specific primers $(\mathrm{M} 13 \mathrm{~F} / \mathrm{R})$ that flank the multiple cloning site. This was then aligned against the relative predicted sequence for each construct. This analysis showed that the overlap PCR had produced the desired truncated gene and that the mutated fragments had been inserted into the plasmid correctly. A list of target genes successfully truncated and cloned into the knockout vector inserts is summarised in below in table 4.2 .

\subsubsection{Absent overlap products}

A total of 14 Psa-V genes

were selected as candidates gene knockout targets

(Table 4.1). However, truncated mutant genes were

Table 4.2: List of target genes successfully truncated and cloned into the knockout vector pEX18-Gm. not successfully generated for all of these targets by

\begin{tabular}{llll}
\hline gacS & gacA & $\mathrm{N} 1$ & $\mathrm{~N} 2$ \\
hopH1 & hrpA & hrpK & hrpL \\
3004 & mtaP & & \\
\hline
\end{tabular}
the overlap extension PCR method. For example, a target gene of particular interest that could not be successfully truncated within the scope and time frame of this project was the type III effector hopZ5 gene. Primer sets were designed as described above and the initial fragments to be overlapped were generated by PCR, but even multiple attempts to optimise the reaction conditions did not produce the desired $2 \mathrm{~kb}$ extension overlap PCR product. One of the candidates shown in the table, $a h l R$, did not have a homologous gene in the Psa-V genome and this gene was considered a candidate only before the whole genome sequence was available.

\subsubsection{Vector selection}

Based on the initial results of the $\mathrm{IC}_{50}$ screening of chloramphenicol and gentamicin, and in the light of previous work by Dr. Jeremy Owen in the Ackerley lab using pDM4 to produce knockout strains in other Pseudomonas spp., this vector was initially chosen for use in the gene knockout study. A further advantage of using the pDM4 vector was that a $P$. syringae pv. phaseolicola 1448a strain that Dr Owen had worked with could be used in parallel to Psa-V knockout experiments as a positive control for certain stages of the multi-step gene knockout procedure (i.e. transformation and integration). However, after working with the pDM4 vector and chloramphenicol selection for some time it was found that spontaneous resistance to chloramphenicol would occur readily in Psa-V. Post electroporation plating of a negative vector control aliquot of electrocompetent Psa- $\mathrm{V}$ would spontaneously yield an average of 10 resistant mutants per $\mu \mathrm{L}$ of electrocompetent cells (an average of 600 resistant mutants per plate in a standard reaction), rendering this selection method ineffective. Instead, pEX18Gm with gentamicin selection replaced pDM4 as the principle integrative knockout vector system. 


\subsubsection{Transformation of Psa-V}

The knockout

vector pEX18-Gm will not replicate in $P$. syringae, and thus only cells that have chromosomally integrated the vector by undergoing homologous recombination with the vector at the target site will carry the selectable gentamicin resistant gene aacC1 (Fig. 4.5). This primary integration is a low frequency event [265].

\subsubsection{Electroporation}

As a positive control to examine the electrocompetency of Psa-V prepared by the method described in chapter 2 , section 2.7.3, it was necessary to select a broad spectrum plasmid that could replicate freely in Psa-V. For this purpose the plasmid pUCP22 was chosen as it will replicate in other P. syringae pathovars and it contains the same selective marker as pEX18-Gm, also pUC plasmids are extensively used to compare transformation efficiencies between methods and strains [266]. When Psa-V was electroporated with the pUCP22 control plasmid, gentamicin resistant colonies were recovered at high frequencies. For example, when electroporated with $100 \mathrm{ng}$ of purified pUCP22 >1000 colonies were recovered from plating $100 \mu \mathrm{L}$ of a 1:10 dilution of a single electroporation reaction and gentamicin gave $100 \%$ selection in control reactions. Plasmid DNA of an equivalent molecular weight to pUCP22, determined by electrophoresis, was extracted from the transformed cells. Similar results were seen when the plasmid pSX was used to transform Psa-V in subsequent experiments of this study (section 4.4). It was concluded from these observations that Psa-V could be made thoroughly electrocompetent using the method described above.

To achieve successful integration of suicide plasmids in $P$. syringae it is typically necessary to introduce a much higher concentration of plasmid DNA than that required for simple transformation with a replicating plasmid. For integrating plasmids, it is recommended that a least $500 \mathrm{ng}$ of plasmid DNA in a volume not exceeding $5 \mu \mathrm{L}$ is added to a single electroporation reaction as plasmid integration is an unstable and low frequency event [265]. For this purpose plasmid DNA was extracted from $50 \mathrm{~mL}$ cultures of E.coli DH5 $\alpha$ by midi-prep to yield concentrations of $500-1500 \mathrm{ng} / \mu \mathrm{L}$, suitable for setting up electroporation reactions of integrating plasmids. Up to $5 \mathrm{\mu g}$ of purified knockout plasmid DNA was added to $60 \mu \mathrm{L}$ aliquots of electrocompetent Psa-V. This mixture was added to a 
$2 \mathrm{~mm}$ electroporation cuvette and pulsed with pre-set parameters. This set up routinely give a suitable pulse duration of $4-5 \mathrm{~ms}$ for the high resistance samples $(<1000 \Omega)$. Failure to maintain high sample resistance normally resulted in electrical arcing across the cuvette, when this occurred samples were disposed of and repeated de novo. Each round of electroporation was also accompanied by negative vector controls to ensure the antibiotic selection was effective.

A small number of gentamicin resistant Psa-V colonies for several targets were recovered during the multitude of repeats using this method of transformation. However, primary integration could not be confirmed by PCR screening. Despite this, the gentamicin resistance gene carried on the knockout plasmid, aacC1, was amplified from these colonies. It was possible that another plasmid carrying aacC1 had cross contaminated the electroporation reactions to give the observed gentamicin resistance and aacC1 positive PCR results. The negative pEX18-Gm::target insert control reactions were free from any signs of cross contamination. The time frame and additional research commitments of the study prevented further investigation into these possible primary integrants; although they have been stored as glycerol cultures at $-80{ }^{\circ} \mathrm{C}$ to enable further analysis at a later date.

\subsubsection{Conjugation}

Biparental and triparental conjugation was also employed in the attempt to generate primary integration of the knockout vector. At the time of writing other groups had successfully used triparental mating to transform Psa-V with pEX18/19 knockout plasmids (Dr Elena Colombi, personal communication, March, 2014) [254]. Although the knockout plasmid used by these groups was the same as that employed for this study, the exact conjugation methods used (i.e. a helper strain carrying the plasmid pRK2013) were not available for the research phase of this work. Neither the bi- nor triparental method used in this study resulted in any detectable plasmid transfer, including experiments that used the mobilisable broad spectrum plasmid pMP190 [267] as a positive conjugation control. The method of triparental conjugation used in this study relied on an uncharacterised helper strain known as AKN98. Following the lack of successful plasmid transfer using AKN98 an alternative was sought. For this purpose the biparental conjugation strain S17-1 was sent to the Ackerley lab by Prof. Iain Lamont, Otago University. Unfortunately, the stock sent to the Ackerley lab was discovered to be contaminated and time constraints prevented any further biparental conjugation experiments.

\section{Initial conjugation optimisation experiments}

For effective recovery of transconjugant colonies, it is essential that the E. coli donor and helper strains be unable to grow on the plating media, otherwise the small minority of Psa-V primary integrant colonies will be obscured and out-competed. At the time that these experiments were initially envisaged, no knowledge of a selective media effective in removing the E. coli donor/helper strains, but allowing Psa-V to thrive, was known.

Several possible selective media options were tested and a continuous effort was made to find the optimal selective method through out this study, including engineering the donor 
strain in an attempt to introduce sensitivity to a selective agent.

Listed below are the different selective media tested in this study, together with the relative characteristics and level of specificity measured for each (Table 4.3) A desirable characteristic of a selective media was maintaining the absolute growth rate of Psa-V. Media that significantly slowed Psa-V growth compared to growth on LB was considered unsatisfactory as these experiments needed to be repeated a number of times. The level of selection was calculated as a percentage of $E$. coli donor strain CFUs found to grow on the selective medium versus LB, and then normalised against the percentage of Psa-V CFUs found to grow on the selective medium versus LB.

Table 4.3: Selective media

\begin{tabular}{|c|c|c|}
\hline Media & Characteristics & Specificity \\
\hline Psa1 & $\begin{array}{l}\text { Pre-formulated selective media designed to } \\
\text { isolate Psa from environmental samples. } \\
\text { Does not store well and cannot be made in } \\
\text { house. Psa-V growth was } 30 \% \text { slower than } \\
\text { on LB agar. }\end{array}$ & $99.5 \%$ selective. \\
\hline Irgasan (Triclosan) & $\begin{array}{l}\text { Antimicrobial agent used in microbiology to } \\
\text { select for pseudomonads especially } P \text {. aerug- } \\
\text { inosa. Psa-V growth was } 60 \% \text { slower than } \\
\text { on LB agar. }\end{array}$ & $40 \%$ selective. \\
\hline Succinate minimal media & $\begin{array}{l}\text { Minimal media to select against auxotrophic } \\
\text { E. coli. Psa-V growth was } 66 \% \text { slower than } \\
\text { on LB agar. }\end{array}$ & $90 \%$ selective. \\
\hline Nitrofurantoin & $\begin{array}{l}\text { Nitrofurantoin is a nitroaromatic antibiotic. } \\
\text { It is activated by nitroreductase enzymes in } \\
E \text {. coli. Psa-V growth on this medium was } \\
\text { equal growth on LB agar. }\end{array}$ & $100 \%$ selective. \\
\hline
\end{tabular}

From the data presented in 4.3 it was concluded that nitrofurantoin is the ideal selective agent to separate the E. colidonor from Psa-V in bacterial mating mixtures.

\section{$E$.coli tolC knockouts \& niclosamide as a selective agent for conjugation}

Before the knowledge that nitrofurantoin would be a useful tool for the differential selection of Psa-V form E. coli was apparent, the lack of a convenient means to remove the E. coli strain(s) from conjugation mixtures led to a series of experiments with the aim of sensitising the E. coli conjugation donor and helper strains available in the lab to niclosamide. As described in chapter 3, section 3.3, in the absence of a TolC inhibitor Psa-V can tolerate niclosamide concentrations of $>200 \mu \mathrm{M}$. When Psa-V was plated on LB agar plates supplemented with $\sim 150 \mu \mathrm{M}$ niclosamide no significant difference in colony numbers or growth rates compared to unsupplemented LB agar were recorded. This lack of sensitivity is thought to be facilitated by membrane spanning substrate/proton antiporter 
efflux pumps that can expel niclosamide and other toxic substrates from the cytoplasm, especially the tolC like family of efflux pumps.

Previous work in the Ackerley lab had shown that a nitroreductase deficient strain of $E$. coli could be sensitised to very low levels of niclosamide by knocking out tolC in this strain (unpublished work), it was postulated that a similar tolC knockout in the E. coli conjugation strains may produce reasonable levels of niclosamide sensitivity in these strains. Sensitising the $E$. coli conjugation strains to niclosamide would allow a means of differential selection of the tolerant $\mathrm{Psa}-\mathrm{V}$ recipient in conjugation experiments.

The method used to knockout E. coli genes is described in chapter 2, section 2.10. Briefly, the method employs phage $\lambda$ Red recombinase mediated homologous recombination to exchange the knockout target gene with a PCR generated kanamycin resistance cassette. This introduced resistance cassette contains FRT (FLP recognition target) sites and can be subsequently removed by introducing a helper plasmid encoding the FLP recombinase.

The tri-parental conjugation strains E. coli DH5 $\alpha$ (donor) and E. coli AKN98 (helper) were generated by exchanging the tolC gene of each strain with the resistance cassette. The exchange was confirmed by PCR screening and the $\lambda$ Red recombinase plasmid was cured by incubating cells at $42{ }^{\circ} \mathrm{C}$ and counter screening for ampicillin sensitivity.

At this point the E. coli tolC knockout strains were tested for niclosamide sensitivity by plating on LB agar $\pm 150 \mu \mathrm{M}$ niclosamide. Neither of the three strains showed any sensitivity to niclosamide in this test. This was thought to be due the protective action of the endogenous nitroreductase in E. coli that detoxify niclosamide. No further work with these strains was carried out from this point as nitrofurantoin has by this stage been shown to be effective in for Psa-V selection.

\subsection{Non-ribosomal peptide synthetase over expression in Psa-V}

One of the knockout targets of interest in Psa-V was a putative non-ribosomal peptide synthetase (NRPS) containing gene cluster that may be involved in producing a phytotoxin or other secondary metabolite that contributes to the virulence of Psa-V. This target was of particular interest as among the known strains of Pseudomonas syringae pv. actinidiae it is unique to the highly virulent outbreak strain Psa-V [20]. A Psa-V knockout of this target may be of use not only for characterising any virulence associated with the NRPS product, but also help interested researchers identify the product itself by acting as a background reference; for example, by comparing a null mutant to wild type Psa-V in a metabolomics analysis, to identify the product(s) missing from the mutant strain. Given the inability to successfully generate targeted Psa-V knockouts within the timeframe of this study, another approach was adopted to produce a strain with a reduced expression of the final NRPS product.

It was hypothesised that over-expression of the first module of the second (two-module) NRPS enzyme in this cluster would disrupt the overall production of the final product. Different NRPS enzymes associate in trans via specific inter-module associations mediated 
by communication-mediating (COM) domains [268]. Thus, it was hypothesised that high-level over-expression of an incomplete single-module variant of the second NRPS enzyme in this cluster would lead to a scenario where most intracellular copies of the first NRPS enzyme were associated with the incomplete version of the second NRPS enzyme, and hence would be unable to make the wild type virulence factor. A similar scenario was previously observed in P. aeruginosa PAO1, where over-expression of an attenuated PvdD pyoverdine synthetase resulted in a major knock-down in the levels of pyoverdine production [269].

The sequence boundaries of this incomplete module were determined by Clustalw alignment against the protein sequence of the first module of the $P$. aeruginosa pyoverdine synthetase PvdD and primers were designed to allow the amplification and cloning of this $\sim 3.3 \mathrm{~kb}$ sequence into the $P$. syringae compatible IPTG regulated expression vector pSX. The insert was ligated into the pSX vector using the restriction sites NdeI and SalI and transformed into chemically competent E. coli DH5 $\alpha$. Transformants were PCR screened and at the time of writing the desired plasmid identity had just been verified by Sanger sequencing. The plasmid has not yet been transformed into Psa-V. However, preliminary experiments using the pSX vector containing an unverified insert of comparable size to the final verified insert indicated that Psa-V can be readily transformed by electroporation with a pSX based plasmid of this size, with transformation efficiencies in the range of $10^{5}$ to $10^{6}$ transformants / $\mu \mathrm{g}$ of plasmid DNA. Thereafter, high-level over-expression of the attenuated (single-module) NRPS protein will be tested by SDS-PAGE analysis. If over-expression is successfully visualized, the plasmid will then be sent to collaborators at Plant \& Food Research Ltd. for a metabolomics analysis (Dr David Ackerley, personal communication). Also, in relation to these experiments pSX containing the kanamycin resistance gene Tn5 neomycin phosphotransferase was constructed. This may be used as a plasmid/expression control in in planta virulence studies of pSX mediated NRPS knock-down strains of Psa-V and doubles as a selection agent in fitness assays between an NRPS knock-down and an NRPS wildtype strain.

\subsection{Discussion}

This chapter describes the efforts undertaken in this study towards producing targeted in-frame gene knockout strains of a highly virulent bacterial kiwifruit pathogen Pseudomonas syringae pv. actinidiae. The initial motivation to develop a knockout platform was to allow avirulent strains to be generated and tested as possible biocontrol agents. The knockout platform incentive was backed up by the expressions of interest from other researchers looking to characterise gene function in Psa-V. Although Psa-V knockouts were not successfully produced in this study, at the time of writing there were recently published [254] reports of successful efforts to produce and study Psa-V knockouts. Given this, it is likely than an adoption of the knowledge and methods used to achieve high-level conjugal transfer of suicide plasmid DNA into Psa-V will allow some of the main aims and goals of this study to be achieved in ongoing efforts.

Mitigating the failure to achieve the desired knockout results in this chapter was the 
identification of Psa-V target genes of interest for knockout work, as well as the production of a selection of truncated targets genes successfully cloned into a knockout vector shown to work in Psa-V. This work should allow the rapid adoption of knockout vector transformation methods in the Ackerley lab.

The published methods that have resulted in Psa-V knockouts (using a wild type Italian isolate) reportedly employed tri-parental mating between E. coli and Psa-V using with the helper strain E. coli DH5 $\alpha$ (pRK2013) [254]. In contrast, tri-parental mating using the helper strain AKN98 in this study failed to yield transformed Psa-V colonies for any of the suicide gene constructs, or for the freely replicating, mobilisable plasmid pMP190 trialed as a positive conjugation control (although it should be noted that this plasmid was not an ideal choice as a positive control as it relies on chloramphenicol resistance as the selective agent).

Equal focus was directed at transforming Psa-V with the integrating knockout plasmids by electroporation in this study as P. syringae pv. phaseolicola 1448a knockouts were previously successfully generated in the Ackerley lab using this method of transformation. This electroporation method, based on a protocol previously described in [126], was found to readily transform Psa- $\mathrm{V}$ with freely replicating (i.e. non-integrating) plasmids, gaining transformation efficiencies in the order of $10^{6}$ transformants / $\mu \mathrm{g}$ of DNA. Coupled with the previously observed suitability for knockout work and the efficiency seen with Psa-V, the electroporation method had a rapid turnaround time, required less labour and fewer resources, and overall had fewer variables (i.e. strains, plasmids and selection steps) compared to conjugation. For these reasons the optimisation of an electroporation method for transforming Psa-V with integrating knockout plasmids was deemed to be valuable to this study. It is possible that primary integrants were successfully achieved using this method, but all attempts at definitively proving a successful integration event by diagnostic PCR failed, except for reactions targeting the aacC1 (gentamicin resistance) selectable marker gene. Thus, it appears instead that some manner of non-homologous integration event may have occurred, leading the Psa-V cells to gain gentamicin resistance in a non-targeted fashion. Ultimately, although some useful progress was made, the end-goal of achieving successful targeted knock out of Psa-V virulence genes was not achieved.

One final observation from this work that may hold significance for other researchers in the field was the poor suitability of chloramphenicol as a selective agent when working with Psa-V. The initial Psa-V knockout experiments were modelled on previous work carried out in P. syringae pv. phaseolicola 1448a, which used the knockout plasmid pDM4 (containing a chloramphenicol resistance gene) to great effect. In contrast, when this plasmid was used to target Psa-V in electroporation experiments, chloramphenicol resistant Psa- $\mathrm{V}$ arose at a rate of $\sim 100$ colonies per reaction in samples electroporated either with or without the plasmid. As such, it was concluded that chloramphenicol as a transformation selection agent should be avoided in Psa-V, especially when high cell densities are to be plated. 


\section{Chapter 5}

\section{Conclusions}

Related strains of Pseudomonas syringae pv. actinidiae displaying a highly virulent phenotype have emerged as agents of sever disease in kiwifruit. The disease caused is capable of killing mature vines and the pathogen has spread quickly to all important kiwifruit growing regions worldwide.

This pathogen and the development of effective management strategies have become the focus of international research efforts.

\subsection{Research motivation}

The identification of chemical compounds that can prevent the infection of kiwifruit by Psa-V has been immense interest to the New Zealand kiwifruit industry due to the lack of protection afforded by the primary method available to growers (i.e. spraying with copper-based solutions) at the time Psa-V was discovered in this country. An urgent response was deemed necessary to safeguard the investments and future profits of the industry here, as reports from Italy described widespread destruction and heavy economic losses caused by the disease. This rapidly led to a need to consider antibiotic options, with a concomitant need to make the ultimate decision as informed as possible, taking into account numerous factors including environmental safety and persistence, phytotoxicity, and sustainability. Thus, one of the primary goals of this study was to compare the efficacy and resistance potential of compounds with anti-Psa-V activity.

In addition to investigating chemical control methods, the opportunity to develop a biological control method for Psa-V was recognised. This was based on research and commercial use of biological agents in controlling other phytopathogenic bacteria and motivated by the possibility of providing a sustainable medium to long-term control option; in essence, buying time for the more sustainable long-term solution of plant breeding to arrive at Psa-V resistant commercial strains.

\subsection{Key findings}

\subsubsection{Measuring anti-Psa-V activity}

The Psa-V inhibitory action of most chemicals can be evaluated by the spectrophotometric microtiter assay optimised in this study. There were three main issues that were found to 
restrict reproducible measurements of a compound by this method. First, a lack of antimicrobial activity towards Psa-V in some cases resulted in measurements that were inconsistent across replicates due in part to the dilution of the growth media by the large volume of test compound needed. When necessary, this was controlled for by using a solution of test compound dissolved in growth media rather than the vehicle, usually DMSO. Ultimately, any compound in this category were considered ineffective for controlling Psa-V and generally of little interest to this study. Second, the precipitation of compounds with very low aqueous solubility was found to interfere with the spectrophotometric analysis. This was overcome in some instances by the addition of a co-solvent which required additional measurements to control for vehicle effects. The third obstacle in measuring Psa-V inhibition was interference due to the strong colour of some test compounds. The majority of test samples to fall into this category were commercial formulations where the colour was an artificial additive.

\subsubsection{Effective compounds}

This study found the antibiotic compounds to be the most effective overall class of compounds tested against $\mathrm{Psa}-\mathrm{V}$, with effective concentrations (i.e. $\mathrm{IC}_{50}$ ) ranging from $<1$ $\mu \mathrm{M}$ to $\sim 50 \mu \mathrm{M}$. This was shadowed by the formal adoption of two aminoglycoside antibiotics, streptomycin and kasugamycin, as chemical control measures in the field. Although these are not the strongest inhibitors of this class tested in this study, other factors preclude the use of the most effective antibiotics to control Psa-V, clinical use in humans being the authoritative factor against field use. There were other compounds found to be effective at concentrations within the same range as the antibiotics. The two silver based compounds were more effective than several of the antibiotics tested, in contrast to this the copper based compounds were found to be almost entirely ineffective against Psa- $\mathrm{V}$ in this assay. Based on this finding, silver may hold some utility as a replacement or supplement for copper as a metal ion based treatment for Psa-V and other phytopathogenic bacteria; although it is possible that cost considerations may preclude large-scale field use. The remaining compounds with strong anti-Psa-V activity were designed as sterilants. Although very strong activity was observed in some of these formulas they may not be appropriate as bactericides applied to plants due to environmental and toxicity issues. To give one example where it is known that in vitro testing does not translate into the field, one potent product tested here was Teracep which is used as a protective fungicide. This product showed high activity against Psa-V in the laboratory but was found to result in negligible protection in vivo [270]. Nonetheless, even if a product is not active on kiwifruit plants, it may still prove useful as an affordable disinfectant to maintain the sterility of orchard equipment in an attempt to reduce the spread of Psa-V throughout and between orchards. Several of the complex mixtures tested in this study are being pursued in this context (Michael Franks, CEO Seeka Kiwifruit Ltd. personal communication). 


\subsubsection{Potentiating the anti-Psa-V activity of niclosamide with an efflux pump inhibitor}

Psa-V growth is not inhibited by the nitroreductase activated antibiotic nitrofurantoin compared to nitroreductase expressing E. coli. Likewise, Psa-V is not protected from the toxic effects of niclosamide when it is also exposed to an efflux pump inhibitor, with researchers in the Ackerley lab having shown that nitroreductase enzymes are able to protect a range of bacteria from this effect. Without an efflux pump inhibitor, niclosamide is ineffective at inhibiting Psa-V growth. Psa-V does have tolC and mexE efflux pump genes, but there is no strong evidence to suggest Psa- $\mathrm{V}$ has homologues to the nitroreductase genes $n f s A$ or $n f s B$. With niclosamide having previously been used in the clinic as a treatment for tape worm parasites [271], and as a molluscicide in fresh water ponds and rice paddies [272], it is likely to be safe for agricultural use.

\subsubsection{Streptomycin resistance}

Streptomycin was registered as a control measure during the initial research phase of this study, but unfortunately some kiwifruit growers in New Zealand used this antibiotic outside of the regulation provisions issued for its use. This caused controversy and increasing political pressure to halt further use of antibiotics in orchards [273, 274]. Nonetheless, before this study was complete, kasugamycin was also registered for PsaV control in kiwifruit orchards.

The potential of Psa-V to develop resistance to streptomycin was tested after this antibiotic was registered for field use. It was found that streptomycin resistance can arise spontaneously in Psa- $\mathrm{V}$ in the lab. This was considered an important finding as streptomycin is the most active anti-Psa-V agent currently available to control Psa-V. If resistance to streptomycin arises in the field the antibiotic control options will be limited to the less potent kasugamycin and this reduction in available antibiotic options may exacerbate resistance arising to any remaining control options.

Preliminary investigations suggest the point mutation in streptomycin resistant Psa-V from this study does not reduce the reproductive fitness of this strain compared to the wildtype. Therefore, once it arises this strain will likely persist in the environment regardless of the pattern of streptomycin use thereafter. One practice that may keep antibiotic resistance in check is the application of both streptomycin and kasugamycin together.

Where antibiotics with different modes of activity, and therefore different resistance mechanism, are used together the likelihood of resistance arising to an individual antibiotic is effectively reduced to zero. The chances of a strain arising with dual resistance, which would require mutations that confer resistance to both antibiotics occurring together in the same cell, has the probability of $P 1 * P 2$, where $P 1$ is the probability of a resistance mutation to one antibiotic occurring at each cell division and $P 2$ to the probability of mutation giving resistance to the other antibiotic occurring at each cell division. As only the cells with resistance to both antibiotics will survive in lethal concentrations of the drugs any cells that develop only a single resistance mutation will not reproduce and a strain 
resistant to a single antibiotic will be very unlikely to arise under dual drug conditions [136].

The potential for Psa- $\mathrm{V}$ to develop resistance to kasugamycin was also tested in this study. Using a similar regime to that was found to rapidly promote streptomycin resistance, it was not possible to recover kasugamycin resistant variants of Psa- $\mathrm{V}$, suggesting that resistance to this antibiotic is likely to develop much more slowly than streptomycin resistance. This is very promising for the use of kasugamycin in the field; but of course does not entirely rule out the possibility of kasugamycin resistance arising. If pressed to interpret the (admittedly still very preliminary) results of this study, my recommendation would be that dual antibiotic use is to be very strongly encouraged; but that if this is not acceptable to the industry, spraying with kasugamycin should be preferred to streptomycin. However, a more detailed follow up study should be performed before any such recommendation is formally made.

Another advantage to using kasugamycin in conjunction with streptomycin is that resistance to kasugamycin is gained through a loss of function mutation. The enzyme KsgA methylase is needed to function in order for kasugamycin to have activity [187]. Importantly, a loss of function mutation like this cannot be carried on a plasmid, so kasugamycin resistance cannot be rapidly acquired by horizontal transfer from another species.

It remains to be seen whether our recommended combination of streptomycin and kasugamycin will ever be trialled in this country.

\subsubsection{Psa-V knockout attempts}

This aspect of this study failed to produce the final desired result of Psa-V knockouts. However, targets of interest to produce viable avirulent knockout strains were identified and knockout plasmids were created for a subset of these targets. Transforming Psa-V by electroporation was validated and optimised, and two selection issue were also resolved in the course of the knock out research. Selection of Psa-V transformants with chloramphenicol was found to be very poor due to spontaneous chloramphenicol resistance arising in untransformed cells. This was alleviated by switching to a plasmid with a gentamicin resistance gene for knockout work. The selection of of Psa-V from a conjugation mixture was ultimately achieved by simply using nitrofurantoin to eliminate the $E$. coli cells used in these mixtures. This is thought to work because Psa-V does not have the nitroreductase activity required to activate nitrofurantoin in to a toxic form, whereas $E$. coli expresses highly active nitroreductase enzymes.

\subsection{Future directions}

This work has highlighted a number of areas where further research might make a valuable contribution toward understanding and/or combating the spread of Psa-V. First and perhaps foremost, an in-depth and definitive analysis of the behaviour of streptomycin resistant Psa-V is urgently required. It was shown here that streptomycin resistance is easily acquired by a single point mutation, and the preliminary conclusion that this mutation does not appear to carry a significant fitness burden and has important 
implications for how streptomycin might best be applied in the field. Should this mechanism of resistance truly be found to carry a negligible burden, the need to apply streptomycin in concert with a second antibiotic becomes all the more imperative as, once raised in the field, resistance is unlikely to ever be lost. The obvious candidate for co-spraying with streptomycin is kasugamycin, and the streptomycin resistance studies detailed above should be paralleled by a more in depth and rigorous investigation into the potential for spontaneous high-level kasugamycin resistance to arise under field-like conditions. Based on this study it appears that, despite requiring higher administration levels than streptomycin, kasugamycin may offer the best long-term prospects for control of Psa-V. Again, it cannot be emphasised too strongly that we have very few plausible antibiotic options available to us, and to offer Psa-V the chance to develop resistance against streptomycin or kasugamycin by spraying either compound in isolation would be reckless in the extreme.

The other research objective that should receive immediate attention is the generation of Psa-V knockouts using the plasmids constructed in this study. Here, I suggest that priority should be given to targeting the unique effector and NRPS genes found in Psa-V as these will most likely yield the most constructive information on the distinctive virulence of this pathogen. The successful generation of Psa-V knockouts in another recent study [254] makes it likely that this objective can be realized in the short term. In parallel, our strategy to over-express an attenuated NRPS enzyme may enable an in-depth metabolomics analysis to deduce the identity of the putative virulence factor encoded by the Psa-V specific NRPS, and this may reveal a unique virulence factor that perhaps contributes to the extreme virulence of Psa-V relative to other Psa strains.

\subsection{Final remarks}

The management of Psa-V in New Zealand and around the world presents a challenge to scientist that must be met with open collaboration and full participation. A theme of knowledge sharing and workload division is obviously present throughout the field. The underling aim of this work was to contribute towards the of common goal of understanding Psa-V to enable better control of the disease. The work presented here may useful in guiding chemical control practices by opening an informed discussion on antibiotic resistance and providing suggestions to expand the available options. It may also serve as a basis for future molecular biology work aimed at understanding the virulence mechanism of Psa-V. 


\section{Bibliography}

[1] Jim MacLoughlin. 2009. URL:

http://zesprikiwi.com/about/history/jim-macloughlin.

[2] FreshFacts. 2014. URL:

http://www.freshfacts.co.nz/file/fresh-facts-2013.pdf.

[3] Food and Agriculture Organization of the United Nations. 2013. URL: http://www.fao.org/.

[4] Today in History. URL: http://www.nzhistory.net.nz/the-chinese-gooseberrybecomes-the-kiwifruit.

[5] Psa information for NZ kiwifruit growers. 2012. URL: http://www.kvh.org.nz/.

[6] Mark Kilgour et al. The key elements of success and failure in the NZ kiwifruit industry. Lincoln University. Agribusiness and Economics Research Unit., 2008.

[7] H Huang and AR Ferguson. "Kiwifruit (Actinidia chinesis and A. deliciosa) plantings and production in China, 2002". In: New Zealand journal of crop and horticultural science 31.3 (2003), pp. 197-202.

[8] James N Moore, JR Ballington Jr, et al. Genetic resources of temperate fruit and nut crops. 290. International Society for Horticultural Science, 1990.

[9] Susan S Hirano and Christen D Upper. "Bacteria in the Leaf Ecosystem with Emphasis on Pseudomonas syringae a Pathogen, Ice Nucleus, and Epiphyte". In: Microbiology and Molecular Biology Reviews 64.3 (2000), pp. 624-653.

[10] Cindy E Morris et al. "The life history of the plant pathogen Pseudomonas syringae is linked to the water cycle". In: The ISME journal 2.3 (2008), pp. 321-334.

[11] JL Vanneste, J Yu, and DA Cornish. "Molecular characterisations of Pseudomonas syringae pv. actinidiae strains isolated from the recent outbreak of bacterial canker on kiwifruit in Italy". In: New Zealand Plant Protection 63 (2010), pp. 7-14.

[12] SS Hirano and CD Upper. "Population Biology and Epidemiology of Pseudomonas Syringae". In: 28 (1 1990), pp. 155-177. DOI:

10.1146/annurev.py.28.090190.001103. URL: http:

//www . annualreviews.org/doi/abs/10.1146/annurev.py . 28.090190.001103.

[13] CT Bull et al. "Comprehensive list of names of plant pathogenic bacteria, 1980-2007". In: Journal of Plant Pathology 92.3 (2010), pp. 551-592.

[14] Dawn L Arnold et al. "Pseudomonas syringae pv. actinidiae pv. phaseolicola: from has beanto supermodel". In: Molecular plant pathology 12.7 (2011), pp. 617-627. 
[15] Louis Gardan, Hussain Shafif, and Patrick AD Grimont. "DNA relatedness among pathovars of $P$. syringae and related bacteria". In: Pseudomonas syringae Pathovars and Related Pathogens. Springer, 1997, pp. 445-448.

[16] Stephan Heeb and Dieter Haas. "Regulatory roles of the GacS/GacA two-component system in plant-associated and other gram-negative bacteria". In: Molecular plant-microbe interactions 14.12 (2001), pp. 1351-1363.

[17] Beatriz Quiñones, Catherine J Pujol, and Steven E Lindow. "Regulation of AHL production and its contribution to epiphytic fitness in Pseudomonas syringae". In: Molecular plant-microbe interactions 17.5 (2004), pp. 521-531.

[18] M Scortichini, U Marchesi, and P Di Prospero. "Genetic relatedness among Pseudomonas avellanae, P. syringae pv. theae and Ps pv. actinidiae, and their identification". In: European Journal of Plant Pathology 108.3 (2002), pp. 269-278.

[19] C Robin Buell et al. "The complete genome sequence of the Arabidopsis and tomato pathogen Pseudomonas syringae pv. tomato DC3000". In: Proceedings of the National Academy of Sciences 100.18 (2003), pp. 10181-10186.

[20] Honour C McCann et al. "Genomic analysis of the kiwifruit pathogen Pseudomonas syringae pv. actinidiae provides insight into the origins of an emergent plant disease". In: PLoS pathogens 9.7 (2013), e1003503.

[21] RA Lelliott, Eve Billing, and AC Hayward. "A determinative scheme for the fluorescent plant pathogenic pseudomonads". In: Journal of Applied Microbiology 29.3 (1966), pp. 470-489.

[22] Yuichi Takikawa et al. "Pseudomonas syringae pv. actinidiae pv. nov.: the causal bacterium of canker of kiwifruit in Japan." In: Annals of the Phytopathological Society of Japan 55.4 (1989), pp. 437-444.

[23] GM Balestra et al. "Current status of bacterial canker spread on kiwifruit in Italy". In: Australasian Plant Disease Notes 4.1 (2009), pp. 34-36.

[24] M Scortichini. "Occurrence of Pseudomonas syringae pv. actinidiae on kiwifruit in Italy". In: Plant Pathology 43.6 (1994), pp. 1035-1038.

[25] P Ferrante and M Scortichini. "Molecular and phenotypic features of Pseudomonas syringae pv. actinidiae isolated during recent epidemics of bacterial canker on yellow kiwifruit (Actinidia chinensis) in central Italy". In: Plant Pathology 59.5 (2010), pp. 954-962.

[26] Kerry R Everett et al. "First report of Pseudomonas syringae pv. actinidiae causing kiwifruit bacterial canker in New Zealand". In: Australasian Plant Disease Notes 6.1 (2011), pp. 67-71.

[27] J Rees-George et al. "Detection of Pseudomonas syringae pv. actinidiae using polymerase chain reaction (PCR) primers based on the 16S-23S rDNA intertranscribed spacer region and comparison with PCR primers based on other gene regions". In: Plant Pathology 59.3 (2010), pp. 453-464. 
[28] J Chapman, R Taylor, and B Alexander. "Second report on characterisation of Pseudomonas syringae pv. actinidiae (Psa) isolates in New Zealand". In: Ministry of Agriculture and Forestry 10 (2011).

[29] Marco Scortichini et al. "Pseudomonas syringae pv. actinidiae: a re-emerging, multi-faceted, pandemic pathogen". In: Molecular plant pathology 13.7 (2012), pp. 631-640.

[30] Margi I Butler et al. "Pseudomonas syringae pv. actinidiae from recent outbreaks of kiwifruit bacterial canker belong to different clones that originated in China". In: PloS one 8.2 (2013), e57464.

[31] Ian Hallett. Microscopic examination of the progression of Psa-V in Gold 3. Plant and Food Research - Pseudomonas syringae pv. actinidiae (Psa) RESEARCH NOTE. Plant and Food Research, 2012. URL: www.kvh.org.nz/vdb/document/1143.

[32] Psa-V: economic impacts coming. Jan. 11, 2011. URL: http://sunlive.co.nz/news/18059-psav-economic-impacts-coming.html.

[33] Glen Greer and Caroline Saunders. "The costs of Psa-V to the New Zealand kiwifruit industry and the wider community". In: (2012).

[34] Simone Marcelletti et al. "Pseudomonas syringae pv. actinidiae draft genomes comparison reveal strain-specific features involved in adaptation and virulence to Actinidia species". In: PLoS One 6.11 (2011), e27297.

[35] JL Vanneste et al. "Presence of Pseudomonas syringae pv. actinidiae, the causal agent of bacterial canker of kiwifruit, on symptomatic and asymptomatic tissues of kiwifruit". In: New Zealand Plant Protection 64 (2011), pp. 241-245.

[36] KVH recommended spray programmepost-harvest to bud break. June 1, 2012. URL: http://www.kvh.org.nz/vdb/document/91185.

[37] Andreas E Voloudakis, Carol L Bender, and Donald A Cooksey. "Similarity between copper resistance genes from Xanthomonas campestris and Pseudomonas syringae". In: Applied and environmental microbiology 59.5 (1993), pp. 1627-1634.

[38] M Nakajima et al. "Nucleotide sequence and organization of copper resistance genes from Pseudomonas syringae pv. actinidiae". In: European journal of plant pathology 110.2 (2004), pp. 223-226.

[39] Callum Kay. Psa and Italian Kiwifruit Orchards. An observation by Callum Kay. KVH. Apr. 4, 2011. URL: http://www.kvh.org.nz/vdb/document/164.

[40] Klaus Rudolph. Pseudomonas syringae pathovars and related pathogens. Vol. 9. Springer, 1997.

[41] JM Young. "Pseudomonas syringae pv. actinidiae in New Zealand". In: Journal of Plant Pathology 94.1sup (2012), S1-5.

[42] Masami Nakajima. "Mechanisms of bactericide resistance in phytopathogenic bacteria". In: Journal of General Plant Pathology 68.3 (2002), pp. 264-264. 
[43] European Commission et al. "Regulation (EC) No 396/2005 of the European Parliament and of the Council of 23 February 2005 on maximum residue levels of pesticides in or on food and feed of plant and animal origin and amending Council Directive 91/414/EEC". In: Official Journal of the European Union 13.03 (2005), p. 2005.

[44] NZ's reputation as kiwifruit exporter hugely damaged. May 28. URL: http://www.nzherald.co.nz/nz/news/article.cfm?c_id=1\&objectid=10809072.

[45] Psa-V Product trialling Summary of Results. Mar. 8, 2012. URL: http://www.kvh.org.nz/vdb/document/138.

[46] Dieter Haas and Geneviève Défago. "Biological control of soil-borne pathogens by fluorescent pseudomonads". In: Nature Reviews Microbiology 3.4 (2005), pp. 307-319.

[47] Bruce G Clare. "Agrobacterium: Biological plant disease control". In: Advanced Engineered Pesticides. (1993), pp. 129-146.

[48] M Wilson and SE Lindow. "Interactions between the biological control agent Pseudomonas fluorescens A 506 and Erwinia amylovora in pear blossoms." In: Phytopathology 83.1 (1993), pp. 117-123.

[49] John L Norelli, Alan L Jones, and Herb S Aldwinckle. "Fire blight management in the twenty-first century: using new technologies that enhance host resistance in apple". In: Plant Disease 87.7 (2003), pp. 756-765.

[50] Donald A Cooksey. "Reduction of infection by Pseudomonas syringae pv. tomato using a nonpathogenic, copper-resistant strain combined with a copper bactericide." In: Phytopathology 78.5 (1988), pp. 601-603.

[51] WJ Janisiewicz and A Marchi. "Control of storage rots on various pear cultivars with a saprophytic strain of Pseudomonas syringae". In: Plant Disease 76.6 (1992), pp. $555-560$.

[52] Monica Höfte et al. "Pyoverdin production by the plant growth beneficial Pseudomonas strain 7NSK2: ecological significance in soil". In: Iron Nutrition and Interactions in Plants. Springer, 1991, pp. 289-297.

[53] Saskia Buysens et al. "Involvement of pyochelin and pyoverdin in suppression of Pythium-induced damping-off of tomato by Pseudomonas aeruginosa 7NSK2." In: Applied and Environmental Microbiology 62.3 (1996), pp. 865-871.

[54] SD Braun and B Völksch. "Characterization of an Inhibitory Strain of Pseudomonas syringae pv. syringae with Potential as a Biocontrol Agent for Bacterial Blight on Soybean". In: Pseudomonas syringae Pathovars and Related Pathogens-Identification, Epidemiology and Genomics. Springer, 2008, pp. 103-109.

[55] Steven E Lindow. "Competitive exclusion of epiphytic bacteria by Ice- Pseudomonas syringae mutants". In: Applied and environmental microbiology 53.10 (1987), pp. 2520-2527. 
[56] Bart PHJ Thomma, Thorsten Nürnberger, and Matthieu HAJ Joosten. "Of PAMPs and effectors: the blurred PTI-ETI dichotomy". In: The Plant Cell Online 23.1 (2011), pp. 4-15.

[57] Thomas Boller. "Chemoperception of microbial signals in plant cells". In: Annual review of plant biology 46.1 (1995), pp. 189-214.

[58] Jonathan DG Jones and Jeffery L Dangl. "The plant immune system". In: Nature 444.7117 (2006), pp. 323-329.

[59] Thomas Boller and Georg Felix. "A renaissance of elicitors: perception of microbe-associated molecular patterns and danger signals by pattern-recognition receptors". In: Annual review of plant biology 60 (2009), pp. 379-406.

[60] Andrea A Gust et al. "Bacteria-derived peptidoglycans constitute pathogen-associated molecular patterns triggering innate immunity in Arabidopsis". In: Journal of Biological Chemistry 282.44 (2007), pp. 32338-32348.

[61] Pascal Bittel and Silke Robatzek. "Microbe-associated molecular patterns (MAMPs) probe plant immunity". In: Current opinion in plant biology 10.4 (2007), pp. $335-341$.

[62] Cyril Zipfel and Georg Felix. "Plants and animals: a different taste for microbes?" In: Current opinion in plant biology 8.4 (2005), pp. 353-360.

[63] Delphine Chinchilla et al. "The Arabidopsis receptor kinase FLS2 binds flg22 and determines the specificity of flagellin perception". In: The Plant Cell Online 18.2 (2006), pp. 465-476.

[64] Cyril Zipfel et al. "Perception of the Bacterial PAMP EF-Tu by the Receptor EFR Restricts Agrobacterium-Mediated Transformation". In: Cell 125.4 (2006), pp. $749-760$.

[65] Kenichi Tsuda and Fumiaki Katagiri. "Comparing signaling mechanisms engaged in pattern-triggered and effector-triggered immunity". In: Current opinion in plant biology 13.4 (2010), pp. 459-465.

[66] Jonathan DG Jones and Jeffery L Dangl. "The plant immune system". In: Nature 444.7117 (2006), pp. 323-329.

[67] Thorsten Nürnberger et al. "Innate immunity in plants and animals: striking similarities and obvious differences". In: Immunological reviews 198.1 (2004), pp. 249-266.

[68] Miguel Angel Torres, Jonathan DG Jones, and Jeffery L Dangl. "Reactive oxygen species signaling in response to pathogens". In: Plant physiology 141.2 (2006), pp. $373-378$.

[69] Jean-Luc Montillet et al. "Fatty acid hydroperoxides and H2O2 in the execution of hypersensitive cell death in tobacco leaves". In: Plant Physiology 138.3 (2005), pp. $1516-1526$.

[70] Chris Lamb and Richard A Dixon. "The oxidative burst in plant disease resistance". In: Annual review of plant biology 48.1 (1997), pp. 251-275. 
[71] Xiong-Yan Chen and Jae-Yean Kim. "Callose synthesis in higher plants". In: Plant signaling $\& 3$ behavior 4.6 (2009), pp. 489-492.

[72] Lourdes Gómez-Gómez, Georg Felix, and Thomas Boller. "A single locus determines sensitivity to bacterial flagellin in Arabidopsis thaliana". In: The Plant Journal 18.3 (1999), pp. 277-284.

[73] Jane Glazebrook. "Genes controlling expression of defense responses in Arabidopsis2001 status". In: Current opinion in plant biology 4.4 (2001), pp. 301-308.

[74] Peer M Schenk et al. "Coordinated plant defense responses in Arabidopsis revealed by microarray analysis". In: Proceedings of the National Academy of Sciences 97.21 (2000), pp. 11655-11660.

[75] Klaus Maleck et al. "The transcriptome of Arabidopsis thaliana during systemic acquired resistance". In: Nature genetics 26.4 (2000), pp. 403-410.

[76] Tsuneaki Asai et al. "MAP kinase signalling cascade in Arabidopsis innate immunity". In: Nature 415.6875 (2002), pp. 977-983.

[77] Paul J Rushton et al. "WRKY transcription factors". In: Trends in plant science 15.5 (2010), pp. 247-258.

[78] Thomas Eulgem et al. "Early nuclear events in plant defence signalling: rapid gene activation by WRKY transcription factors". In: The EMBO Journal 18.17 (1999), pp. 4689-4699.

[79] Ralph Panstruga, Jane E Parker, and Paul Schulze-Lefert. "SnapShot: plant immune response pathways". In: Cell 136.5 (2009), 978-e1.

[80] Corné MJ Pieterse et al. "Networking by small-molecule hormones in plant immunity". In: Nature Chemical Biology 5.5 (2009), pp. 308-316.

[81] Kenichi Tsuda et al. "Interplay between MAMP-triggered and SA-mediated defense responses". In: The Plant Journal 53.5 (2008), pp. 763-775.

[82] WE Durrant and X Dong. "Systemic acquired resistance". In: Annu. Rev. Phytopathol. 42 (2004), pp. 185-209.

[83] A Corina Vlot, Daniel F Klessig, and Sang-Wook Park. "Systemic acquired resistance: the elusive signal(s)". In: Current opinion in plant biology 11.4 (2008), pp. $436-442$.

[84] Tanja Petnicki-Ocwieja et al. "Genomewide identification of proteins secreted by the Hrp type III protein secretion system of Pseudomonas syringae pv. tomato DC3000". In: Proceedings of the National Academy of Sciences 99.11 (2002), pp. 7652-7657.

[85] Oliver Schraidt et al. "Topology and organization of the Salmonella typhimurium type III secretion needle complex components". In: PLoS pathogens 6.4 (2010), e1000824.

[86] Stephen T Chisholm et al. "Host-microbe interactions: shaping the evolution of the plant immune response". In: Cell 124.4 (2006), pp. 803-814. 
[87] Sarah R Grant et al. "Subterfuge and manipulation: type III effector proteins of phytopathogenic bacteria". In: Annu. Rev. Microbiol. 60 (2006), pp. 425-449.

[88] Robert B Abramovitch, Jeffrey C Anderson, and Gregory B Martin. "Bacterial elicitation and evasion of plant innate immunity". In: Nature Reviews Molecular Cell Biology 7.8 (2006), pp. 601-611.

[89] James R Alfano and Alan Collmer. "The type III (Hrp) secretion pathway of plant pathogenic bacteria: trafficking harpins, Avr proteins, and death." In: Journal of bacteriology 179.18 (1997), p. 5655.

[90] Paula Hauck, Roger Thilmony, and Sheng Yang He. "A Pseudomonas syringae type III effector suppresses cell wall-based extracellular defense in susceptible Arabidopsis plants". In: Proceedings of the National Academy of Sciences 100.14 (2003), pp. $8577-8582$.

[91] Jing Yuan and Sheng Yang He. "The Pseudomonas syringae Hrp regulation and secretion system controls the production and secretion of multiple extracellular proteins." In: Journal of bacteriology 178.21 (1996), pp. 6399-6402.

[92] Jie Zhang et al. "A Pseudomonas syringae pv. actinidiae Effector Inactivates MAPKs to Suppress PAMP-Induced Immunity in Plants". In: Cell Host $\&$ Microbe 1.3 (2007), pp. $175-185$.

[93] Hongtao Li et al. "The phosphothreonine lyase activity of a bacterial type III effector family". In: Science 315.5814 (2007), pp. 1000-1003.

[94] Ping He et al. "Specific Bacterial Suppressors of MAMP Signaling Upstream of MAPKKK in Arabidopsis Innate Immunity". In: Cell 125.3 (2006), pp. 563-575.

[95] Thomas Boller and Sheng Yang He. "Innate immunity in plants: an arms race between pattern recognition receptors in plants and effectors in microbial pathogens". In: Science (New York, NY) 324.5928 (2009), p. 742.

[96] Luis Da Cunha, Mysore-Venkatarau Sreerekha, and David Mackey. "Defense suppression by virulence effectors of bacterial phytopathogens". In: Current opinion in plant biology 10.4 (2007), pp. 349-357.

[97] Erik A Van Der Biezen and Jonathan DG Jones. "Plant disease-resistance proteins and the gene-for-gene concept". In: Trends in biochemical sciences 23.12 (1998), pp. $454-456$.

[98] Harold H Flor. "Current status of the gene-for-gene concept". In: Annual review of phytopathology 9.1 (1971), pp. 275-296.

[99] Timothy K Eitas and Jeffery L Dangl. "NB-LRR proteins: pairs, pieces, perception, partners, and pathways". In: Current opinion in plant biology 13.4 (2010), pp. $472-477$.

[100] NS Coll, P Epple, and JL Dangl. "Programmed cell death in the plant immune system". In: Cell Death $\&$ Differentiation 18.8 (2011), pp. 1247-1256. 
[101] Saikat Bhattacharjee, Christopher M Garner, and Walter Gassmann. "New clues in the nucleus: transcriptional reprogramming in effector-triggered immunity". In: Frontiers in plant science 4 (2013).

[102] Fumiaki Katagiri and Kenichi Tsuda. "Understanding the plant immune system". In: Molecular plant-microbe interactions 23.12 (2010), pp. 1531-1536.

[103] Walter Gassmann and Saikat Bhattacharjee. "Effector-triggered immunity signaling: from gene-for-gene pathways to protein-protein interaction networks". In: Molecular Plant-Microbe Interactions 25.7 (2012), pp. 862-868.

[104] Jun Liu et al. "A receptor-like cytoplasmic kinase phosphorylates the host target RIN4, leading to the activation of a plant innate immune receptor". In: Cell host $E$ microbe 9.2 (2011), pp. 137-146.

[105] Koji Yamaguchi et al. "A Receptor-like Cytoplasmic Kinase Targeted by a Plant Pathogen Effector Is Directly Phosphorylated by the Chitin Receptor and Mediates Rice Immunity". In: Cell host \&3 microbe 13.3 (2013), pp. 347-357.

[106] Zhiyong Gao et al. "Plant intracellular innate immune receptor Resistance to Pseudomonas syringae pv. maculicola 1 (RPM1) is activated at, and functions on, the plasma membrane". In: Proceedings of the National Academy of Sciences 108.18 (2011), pp. 7619-7624.

[107] Nuria S Coll et al. "Arabidopsis type I metacaspases control cell death". In: Science 330.6009 (2010), pp. 1393-1397.

[108] PV Bozhkov and E Lam. "Green death: revealing programmed cell death in plants". In: Cell Death $\&$ Differentiation 18.8 (2011), pp. 1239-1240.

[109] WG Van Doorn et al. "Morphological classification of plant cell deaths". In: Cell Death \& Differentiation 18.8 (2011), pp. 1241-1246.

[110] Dawn L Arnold and Robert W Jackson. "Bacterial genomes: evolution of pathogenicity". In: Current opinion in plant biology 14.4 (2011), pp. 385-391.

[111] Sara F Sarkar and David S Guttman. "Evolution of the core genome of Pseudomonas syringae, a highly clonal, endemic plant pathogen". In: Applied and Environmental Microbiology 70.4 (2004), pp. 1999-2012.

[112] Hiroyuki Sawada et al. "Phylogenetic analysis of Pseudomonas syringae pathovars suggests the horizontal gene transfer of $\arg K$ and the evolutionary stability of hrp gene cluster". In: Journal of Molecular Evolution 49.5 (1999), pp. 627-644.

[113] Caroline L Monteil et al. "Nonagricultural reservoirs contribute to emergence and evolution of Pseudomonas syringae crop pathogens". In: New Phytologist (2013).

[114] James R Alfano et al. "The Pseudomonas syringae Hrp pathogenicity island has a tripartite mosaic structure composed of a cluster of type III secretion genes bounded by exchangeable effector and conserved effector loci that contribute to parasitic fitness and pathogenicity in plants". In: Proceedings of the National Academy of Sciences 97.9 (2000), pp. 4856-4861. 
[115] Dawn L Arnold et al. "Evolution of microbial virulence: the benefits of stress". In: TRENDS in Genetics 23.6 (2007), pp. 293-300.

[116] Donald A Cooksey. "Plasmid-determined copper resistance in Pseudomonas syringae from impatiens". In: Applied and environmental microbiology 56.1 (1990), pp. 13-16.

[117] Hiroyuki Sawada, Toru Takeuchi, and Izumi Matsuda. "Comparative analysis of Pseudomonas syringae pv. actinidiae and pv. phaseolicola based on phaseolotoxin-resistant ornithine carbamoyltransferase gene $(\arg K)$ and $16 \mathrm{~S}-23 \mathrm{~S}$ rRNA intergenic spacer sequences." In: Applied and environmental microbiology 63.1 (1997), pp. 282-288.

[118] Andrew R Pitman et al. "Exposure to host resistance mechanisms drives evolution of bacterial virulence in plants". In: Current biology 15.24 (2005), pp. 2230-2235.

[119] Angelo Mazzaglia et al. "Pseudomonas syringae pv. actinidiae (PSA) isolates from recent bacterial canker of kiwifruit outbreaks belong to the same genetic lineage". In: PLoS One 7.5 (2012), e36518.

[120] GM Balestra et al. "A multiplex PCR assay for detection of Pseudomonas syringae pv. actinidiae and differentiation of populations with different geographic origin". In: Plant Disease 97.4 (2013), pp. 472-478.

[121] Susana De la Torre-Zavala et al. "Gene expression of Pht cluster genes and a putative non-ribosomal peptide synthetase required for phaseolotoxin production is regulated by GacS/GacA in Pseudomonas syringae pv. phaseolicola". In: Research in microbiology 162.5 (2011), pp. 488-498.

[122] JL Vanneste et al. "Presence of the effector gene hopA1 in strains of Pseudomonas syringae pv. actinidiae isolated from France and Italy". In: New Zealand Plant Protection 64 (2011), pp. 252-258.

[123] Sara F Sarkar et al. "Comparative genomics of host-specific virulence in Pseudomonas syringae". In: Genetics 174.2 (2006), pp. 1041-1056.

[124] Jeremy G Owen and David F Ackerley. "Characterization of pyoverdine and achromobactin in Pseudomonas syringae pv. phaseolicola 1448a". In: BMC microbiology 11.1 (2011), p. 218.

[125] Jeremy Owen. "Characterisation, manipulation and directed evolution of non-ribosomal peptide synthetase enzymes". phD. Victoria University Wellington, 2010 .

[126] Kyoung-Hee Choi, Ayush Kumar, and Herbert P. Schweizer. "A 10-min method for preparation of highly electrocompetent Pseudomonas aeruginosa cells: Application for DNA fragment transfer between chromosomes and plasmid transformation". In: Journal of Microbiological Methods 64 (3 2006), 391397. (Visited on 12/02/2013).

[127] Kirill A. Datsenko and Barry L. Wanner. "One-step inactivation of chromosomal genes in Escherichia coli K-12 using PCR products". In: Proceedings of the National Academy of Sciences 97 (12 2000), 66406645. 
[128] Ulrich K Laemmli et al. "Cleavage of structural proteins during the assembly of the head of bacteriophage T4". In: nature 227.5259 (1970), pp. 680-685.

[129] Joseph Sambrook, David W Russell, and David W Russell. Molecular cloning: a laboratory manual (3-volume set). Cold Spring Harbor Laboratory Press Cold Spring Harbor New York, 2001.

[130] D Steven et al. "Changing pest control on New Zealand kiwifruit". In: III International Symposium on Kiwifruit 444. 1995, pp. 765-772.

[131] PR Sale, MJ Hartley, et al. "The history of pest and disease control in kiwifruit." In: Proceedings of the thirty-third New Zealand weed and pest control conference. Willow Park Motor Hotel, Tauranga, August 12th to 14th, 1980. New Zealand Weed and Pest Control Society Inc. 1980, pp. 110-113.

[132] Young Jin Koh et al. "Chemical and cultural control of bacterial blossom blight of kiwifruit caused by Pseudomonas syringae in Korea". In: New Zealand Journal of Crop and Horticultural Science 29.1 (2001), pp. 29-34.

[133] K Ushiyama. "Studies on the epidemics and control of bacterial canker of kiwifruit (Actinidia deliciosa) caused by Pseudomonas syringae pv. actinidiae". In: Bulletin of the Kanagawa Horticultural Experiment Station (1993).

[134] JL Vanneste. "Streptomycin management strategy". In: Pesticide resistance strategies, New Zealand Plant Protection Society Inc. 30.06 (2004), p. 2006.

[135] JL Vanneste and MD Voyle. "Characterisation of transposon, genes and mutations which confer streptomycin resistance in bacterial strains isolated from New Zealand orchards". In: IX International Workshop on Fire Blight 590. 2001, pp. 493-495.

[136] JL Vanneste. Fire blight: the disease and its causative agent, Erwinia amylovora. CABI, 2000.

[137] Young Jin Koh et al. "Chemical and cultural control of bacterial blossom blight of kiwifruit caused by Pseudomonas syringae in Korea". In: New Zealand Journal of Crop and Horticultural Science 29.1 (2001), pp. 29-34.

[138] EPA Submission Form - To import Kasumin 2L bactericide antibiotic containing kasugamycin for the control of Pseudomonas syringe pv. actinidiae (Psa-V) in kiwifuit. URL: http://www.epa.govt.nz/search-databases/HSNO\%20Application\% 20Register\%20Documents/APP201581_SUBMISSION102969.pdf.

[139] JE Adaskaveg, H Förster, and ML Wade. "Effectiveness of kasugamycin against Erwinia amylovora and its potential use for managing fire blight of pear". In: Plant Disease 95.4 (2011), pp. 448-454.

[140] R Onorato et al. "Recent advances in the characterisation and control of Pseudomonas syringae pv. actinidiae, the causal agent of bacterial canker on kiwifruit". In: VII International Symposium on Kiwifruit 913. 2010, pp. 443-455.

[141] David W Wareham and Peter Wilson. "Chloramphenicol in the 21st century". In: British Journal of Hospital Medicine 63.3 (2002), pp. 157-161. 
[142] E Arms and WL Dibb. "Otitis externa: clinical comparison of local ciprofloxacin versus local oxytetracycline, polymyxin B, hydrocortisone combination treatment". In: Current Medical Research and Opinion 13.3 (1993), pp. 182-186.

[143] AD Russell et al. "Antimicrobial Activity and Action of silver". In: Progress in Medicinal Chemistry 31 (1994), p. 351.

[144] HJ Lee, SY Yeo, and SH Jeong. "Antibacterial effect of nanosized silver colloidal solution on textile fabrics". In: Journal of Materials Science 38.10 (2003), pp. 2199-2204.

[145] Steven Pao, D Frank Kelsey, and Wilbert Long. "Spray washing of tomatoes with chlorine dioxide to minimize Salmonella on inoculated fruit surfaces and cross-contamination from revolving brushes". In: Journal of Food Protection 72.12 (2009), pp. 2448-2452.

[146] João PS Cabral. "Mode of antibacterial action of dodine (dodecylguanidine monoacetate) in Pseudomonas syringae". In: Canadian Journal of Microbiology 38.2 (1992), pp. 115-123.

[147] Serge Ankri and David Mirelman. "Antimicrobial properties of allicin from garlic". In: Microbes and infection 1.2 (1999), pp. 125-129.

[148] Christopher J Adams, Merilyn Manley-Harris, and Peter C Molan. "The origin of methylglyoxal in New Zealand manuka (Leptospermum scoparium) honey". In: Carbohydrate research 344.8 (2009), pp. 1050-1053.

[149] Rabia Bourkiza et al. "Initial treatment of Pseudomonas aeruginosa contact lens-associated keratitis with topical chloramphenicol, and effect on outcome". In: British Journal of Ophthalmology 97.4 (2013), pp. 429-432.

[150] ML Avent et al. "Current use of aminoglycosides: indications, pharmacokinetics and monitoring for toxicity". In: Internal medicine journal 41.6 (2011), pp. 441-449.

[151] J Spicer. Therapeutic Guidelines: Antibiotic. Therapeutic guidelines. Therapeutic Guidelines Limited, 2010. ISBN: 9780980476477. URL: http://books . google.co.nz/books?id=hymASgAACAAJ.

[152] Psa Product trialling Summary of Results. URL: http://www.kvh.org.nz/vdb/document/138.

[153] Product screening test for Kasumin and Phyton. URL: http://www.kvh.org.nz/vdb/document/1141.

[154] Ruth Francis-Floyd et al. "Use of Bayluscide (Bayer 73) for snail control in fish ponds". In: Journal of Aquatic Animal Health 9.1 (1997), pp. 41-48.

[155] World Health Organization. WHO SPECIFICATIONS AND EVALUATIONS FOR PUBLIC HEALTH PESTICIDES NICLOSAMIDE 2,5-dichloro-4-nitrosalicylanilide. Tech. rep. Geneva, 2003. URL: http://www. who.int/whopes/quality/en/Niclosamide.pdf (visited on 02/02/2014). 
[156] Francesco Imperi et al. "New life for an old drug: the anthelmintic drug niclosamide inhibits Pseudomonas aeruginosa quorum sensing". In: Antimicrobial agents and chemotherapy 57.2 (2013), pp. 996-1005.

[157] Gareth A Prosser et al. "Creation and screening of a multi-family bacterial oxidoreductase library to discover novel nitroreductases that efficiently activate the bioreductive prodrugs CB1954 and PR-104A". In: Biochemical pharmacology 85.8 (2013), pp. 1091-1103.

[158] SO Vass et al. "E. coli NfsA: an alternative nitroreductase for prodrug activation gene therapy in combination with CB1954". In: British journal of cancer 100.12 (2009), pp. 1903-1911.

[159] Srinivas S Sastry and R Jayaraman. "Nitrofuratoin-resistant mutants of Escherichia coli: Isolation and mapping". In: Molecular and General Genetics MGG 196.2 (1984), pp. 379-380.

[160] MA Webber and LJV Piddock. "The importance of efflux pumps in bacterial antibiotic resistance". In: Journal of Antimicrobial Chemotherapy 51.1 (2003), pp. 9-11.

[161] Xian-Zhi Li and Hiroshi Nikaido. "Efflux-mediated drug resistance in bacteria: an update". In: Drugs 69.12 (2009), p. 1555.

[162] Thomas E Renau et al. "Inhibitors of Efflux Pumps in Pseudomonas aeruginosa Potentiate the Activity of the Fluoroquinolone Antibacterial Levofloxacin". In: Journal of medicinal chemistry 42.24 (1999), pp. 4928-4931.

[163] Olga Lomovskaya et al. "Identification and characterization of inhibitors of multidrug resistance efflux pumps in Pseudomonas aeruginosa: novel agents for combination therapy". In: Antimicrobial Agents and Chemotherapy 45.1 (2001), pp. 105-116.

[164] Ryan P Lamers, Joseph F Cavallari, and Lori L Burrows. "The efflux inhibitor phenylalanine-arginine beta-naphthylamide (PA $\beta \mathrm{N})$ permeabilizes the outer membrane of gram-negative bacteria". In: PloS one 8.3 (2013), e60666.

[165] Saul B Needleman and Christian D Wunsch. "A general method applicable to the search for similarities in the amino acid sequence of two proteins". In: Journal of molecular biology 48.3 (1970), pp. 443-453.

[166] Patricia S McManus et al. "Antibiotic use in plant agriculture". In: Annual Review of Phytopathology 40.1 (2002), pp. 443-465.

[167] Annie Guiyoule et al. "Transferable plasmid-mediated resistance to streptomycin in a clinical isolate of Yersinia pestis." In: Emerging infectious diseases 7.1 (2001), p. 43.

[168] Hyo Shim Han et al. "Molecular bases of high-level streptomycin resistance in Pseudomonas marginalis and Pseudomonas syringae pv. actinidiae". In: Journal of microbiology-Seoul 41.1 (2003), pp. 16-21.

[169] Daniel N Wilson. "The AZ of bacterial translation inhibitors". In: Critical Reviews in Biochemistry and Molecular Biology 44.6 (2009), pp. 393-433. 
[170] Daniel N Wilson. "Ribosome-targeting antibiotics and mechanisms of bacterial resistance". In: Nature Reviews Microbiology 12.1 (2014), pp. 35-48.

[171] Maria S Ramirez and Marcelo E Tolmasky. "Aminoglycoside modifying enzymes". In: Drug Resistance Updates 13.6 (2010), pp. 151-171.

[172] Philip N Rather. "Origins of the aminoglycoside modifying enzymes". In: Drug Resistance Updates 1.5 (1998), pp. 285-291.

[173] GW Sundin and CL Bender. "Ecological and genetic analysis of copper and streptomycin resistance in Pseudomonas syringae pv. syringae." In: Applied and environmental microbiology 59.4 (1993), pp. 1018-1024.

[174] Peter Scholz et al. "Complete nucleotide sequence and gene organization of the broad-host-range plasmid RSF1010". In: Gene 75.2 (1989), pp. 271-288.

[175] Anne ML Barnard et al. "Mutations in rpsL that confer streptomycin resistance show pleiotropic effects on virulence and the production of a carbapenem antibiotic in Erwinia carotovora". In: Microbiology 156.4 (2010), pp. 1030-1039.

[176] Andrew P Carter et al. "Functional insights from the structure of the 30S ribosomal subunit and its interactions with antibiotics". In: Nature 407.6802 (2000), pp. $340-348$.

[177] JL Vanneste and MD Voyle. "Genetic basis of streptomycin resistance in pathogenic and epiphytic bacteria isolated in apple orchards in New Zealand". In: VIII International Workshop on Fire Blight 489. 1998, pp. 671-672.

[178] Chien-Shun Chiou and AL Jones. "Molecular analysis of high-level streptomycin resistance in Erwinia amylovora". In: Phytopathology 85.3 (1995), pp. 324-328.

[179] Adrian Ponce de León Door, Alejandro Romo Chacón, and Carlos Acosta Muñiz. "Detection of streptomycin resistance in Erwinia amylovora strains isolated from apple orchards in Chihuahua, Mexico". In: European Journal of Plant Pathology 137.2 (2013), pp. 223-229.

[180] Betzaida Cuevas-Córdoba et al. "rrs and rpsL mutations in streptomycin-resistant isolates of Mycobacterium tuberculosis from Mexico". In: Journal of Microbiology, Immunology and Infection 46.30 (2013), e34.

[181] Srinand Sreevatsan et al. "Characterization of rpsL and rrs mutations in streptomycin-resistant Mycobacterium tuberculosis isolates from diverse geographic localities." In: Antimicrobial agents and chemotherapy 40.4 (1996), pp. 1024-1026.

[182] Jaygopal Nair et al. "The rpsL gene and streptomycin resistance in single and multiple drug-resistant strains of Mycobacterium tuberculosis". In: Molecular microbiology 10.3 (1993), pp. 521-527.

[183] H Ward, GG Perron, and RC Maclean. "The cost of multiple drug resistance in Pseudomonas aeruginosa". In: Journal of evolutionary biology 22.5 (2009), pp. 997-1003. 
[184] Antón Vila-Sanjurjo, Catherine L Squires, and Albert E Dahlberg. "Isolation of kasugamycin resistant mutants in the $16 \mathrm{~S}$ ribosomal RNA of Escherichia coli". In: Journal of molecular biology 293.1 (1999), pp. 1-8.

[185] Frank Schluenzen et al. "The antibiotic kasugamycin mimics mRNA nucleotides to destabilize tRNA binding and inhibit canonical translation initiation". In: Nature structural \& molecular biology 13.10 (2006), pp. 871-878.

[186] P Frederick Sparling. "Kasugamycin resistance: 30S ribosomal mutation with an unusual location on the Escherichia coli chromosome". In: Science 167.3914 (1970), pp. $56-58$.

[187] Terry L Helser, Julian E Davies, and James E Dahlberg. "Mechanism of kasugamycin resistance in Escherichia coli". In: Nature 235.53 (1972), pp. 6-9.

[188] Robin Ray Gutell. "Collection of small subunit (16S-and 16S-like) ribosomal RNA structures." In: Nucleic acids research 21.13 (1993), p. 3051.

[189] Keith Connolly, Jason P Rife, and Gloria Culver. "Mechanistic insight into the ribosome biogenesis functions of the ancient protein KsgA". In: Molecular microbiology 70.5 (2008), pp. 1062-1075.

[190] Atsushi Yoshii, Hiromitsu Moriyama, and Toshiyuki Fukuhara. "The Novel Kasugamycin 2-N-Acetyltransferase Gene aac (2)-IIa, Carried by the IncP Island, Confers Kasugamycin Resistance to Rice-Pathogenic Bacteria". In: Applied and environmental microbiology 78.16 (2012), pp. 5555-5564.

[191] GE Wang et al. "Spontaneous mutations that confer antibiotic resistance inHelicobacter pylori". In: Antimicrobial agents and chemotherapy 45.3 (2001), pp. 727-733.

[192] Brent Ewing and Phil Green. "Base-calling of automated sequencer traces using phred. II. Error probabilities". In: Genome research 8.3 (1998), pp. 186-194.

[193] Dan I Andersson. "Persistence of antibiotic resistant bacteria". In: Current opinion in microbiology 6.5 (2003), pp. 452-456.

[194] Rouven Bingel-Erlenmeyer et al. "A peptide deformylase-ribosome complex reveals mechanism of nascent chain processing". In: Nature 452.7183 (2008), pp. 108-111.

[195] Dan I Andersson and Bruce R Levin. "The biological cost of antibiotic resistance". In: Current opinion in microbiology 2.5 (1999), pp. 489-493.

[196] Dan I Andersson. "The biological cost of mutational antibiotic resistance: any practical conclusions?" In: Current opinion in microbiology 9.5 (2006), pp. 461-465.

[197] Daniel Criswell et al. "Mutations conferring aminoglycoside and spectinomycin resistance in Borrelia burgdorferi". In: Antimicrobial agents and chemotherapy 50.2 (2006), pp. $445-452$.

[198] BR Levin. "Models for the spread of resistant pathogens." In: The Netherlands journal of medicine 60.7 Suppl (2002), pp. 58-64. 
[199] AJ O'Neill et al. "Molecular genetic and structural modeling studies of Staphylococcus aureus RNA polymerase and the fitness of rifampin resistance genotypes in relation to clinical prevalence". In: Antimicrobial agents and chemotherapy 50.1 (2006), pp. 298-309.

[200] Johanna Björkman, Diarmaid Hughes, and Dan I Andersson. "Virulence of antibiotic-resistant Salmonella typhimurium". In: Proceedings of the National Academy of Sciences 95.7 (1998), pp. 3949-3953.

[201] Sophie Maisnier-Patin et al. "Compensatory adaptation to the deleterious effect of antibiotic resistance in Salmonella typhimurium”. In: Molecular microbiology 46.2 (2002), pp. $355-366$.

[202] AP Davies et al. "Comparison of Fitness of Two Isolates of Mycobacterium tuberculosis, one of Which had Developed Multi-drug Resistance During the Course of Treatment". In: Journal of Infection 41.2 (2000), pp. 184-187.

[203] Li Ren, M Sayeedur Rahman, and M Zafri Humayun. "Escherichia coli cells exposed to streptomycin display a mutator phenotype". In: Journal of bacteriology 181.3 (1999), pp. 1043-1044.

[204] R Jayaraman. "Phase variation and adaptation in bacteria: A'Red Queen's Race". In: Current Science 100.8 (2011), pp. 1163-1171.

[205] Bruce R Levin, Véronique Perrot, and Nina Walker. "Compensatory mutations, antibiotic resistance and the population genetics of adaptive evolution in bacteria". In: Genetics 154.3 (2000), pp. 985-997.

[206] J Björkman et al. "Effects of environment on compensatory mutations to ameliorate costs of antibiotic resistance". In: Science 287.5457 (2000), pp. 1479-1482.

[207] Seasonal Management Guide. 2014. URL: http://www.kvh.org.nz/vdb/document/91494.

[208] Psa-V Product Testing Field Trial Reports. 2012. URL: http://www.kvh.org.nz/vdb/document/91137.

[209] Richard D Pearson and Erik L Hewlett. "Niclosamide therapy for tapeworm infections". In: Annals of internal medicine 102.4 (1985), pp. 550-551.

[210] Christina Kourtesi et al. "Suppl 1: Microbial Efflux Systems and Inhibitors: Approaches to Drug Discovery and the Challenge of Clinical Implementation". In: The open microbiology journal 7 (2013), p. 34.

[211] Aurélie Lieutaud et al. "Inhibitors of Antibiotic Efflux by AcrAB-TolC in Enterobacter aerogenes". In: Anti-Infective Agents 11.2 (2013), pp. 168-178.

[212] Olga Lomovskaya and Keith A Bostian. "Practical applications and feasibility of efflux pump inhibitors in the clinica vision for applied use". In: Biochemical pharmacology 71.7 (2006), pp. 910-918.

[213] Steven E Lindow and Maria T Brandl. "Microbiology of the phyllosphere". In: Applied and environmental microbiology 69.4 (2003), pp. 1875-1883. 
[214] JP Blakeman and NJ Fokkema. "Potential for biological control of plant diseases on the phylloplane". In: Annual Review of Phytopathology 20.1 (1982), pp. 167-190.

[215] Deborah R Fravel. "Role of antibiosis in the biocontrol of plant diseases". In: Annual Review of Phytopathology 26.1 (1988), pp. 75-91.

[216] M.J. Bailey. Microbial Ecology of Aerial Plant Surfaces. CAB books. CABI, 2006. ISBN: 9781845930615. URL: http://books.google.co.nz/books?id=AbydK87m7doC.

[217] R James Cook. "Making greater use of introduced microorganisms for biological control of plant pathogens". In: Annual Review of Phytopathology 31.1 (1993), pp. $53-80$.

[218] Joseph W Kloepper et al. "Pseudomonas siderophores: a mechanism explaining disease-suppressive soils". In: Current microbiology 4.5 (1980), pp. 317-320.

[219] P Narayanasamy. "Mechanisms of Action of Bacterial Biological Control Agents". In: Biological Management of Diseases of Crops. Springer, 2013, pp. 295-429.

[220] Brion K Duffy and Geneviève Défago. "Environmental Factors Modulating Antibiotic and Siderophore Biosynthesis by Pseudomonas fluorescens Biocontrol Strains". In: Applied and Environmental Microbiology 65.6 (1999), pp. 2429-2438.

[221] David Gottlieb. "The production and role of antibiotics in soil." In: The Journal of antibiotics 29.10 (1976), p. 987.

[222] Leland S Pierson et al. "Molecular analysis of genes encoding phenazine biosynthesis in the biological control bacterium Pseudomonas aureofaciens 30-84". In: FEMS microbiology letters 134.2-3 (1995), pp. 299-307.

[223] JM Meyer, and MA Abdallah. "The fluorescent pigment of Pseudomonas fluorescens: biosynthesis, purification and physicochemical properties". In: Journal of General Microbiology 107.2 (1978), pp. 319-328.

[224] PTW Wong and R Baker. "Suppression of wheat take-all and Ophiobolus patch by fluorescent pseudomonads from a Fusarium-suppressive soil". In: Soil Biology and Biochemistry 16.4 (1984), pp. 397-403.

[225] Annette Wensing et al. "Impact of siderophore production by Pseudomonas syringae pv. syringae 22d/93 on epiphytic fitness and biocontrol activity against Pseudomonas syringae pv. glycinea 1a/96". In: Applied and environmental microbiology 76.9 (2010), pp. 2704-2711.

[226] R. May, B. Vlksch, and G. Kampmann. "Antagonistic Activities of Epiphytic Bacteria from Soybean Leaves against Pseudomonas syringae pv. glycinea in vitro and in planta". English. In: Microbial Ecology 34.2 (1997), pp. 118-124. ISSN: 00953628. URL: http://www.jstor.org/stable/4251514.

[227] B Völksch and R May. "Biological control of Pseudomonas syringae pv. glycinea by epiphytic bacteria under field conditions". In: Microbial ecology 41.2 (2001), pp. $132-139$.

[228] Michael E Hibbing et al. "Bacterial competition: surviving and thriving in the microbial jungle". In: Nature Reviews Microbiology 8.1 (2010), pp. 15-25. 
[229] Emma Barahona et al. "Pseudomonas fluorescens F113 mutant with enhanced competitive colonization ability and improved biocontrol activity against fungal root pathogens". In: Applied and environmental microbiology 77.15 (2011), pp. 5412-5419.

[230] Marsilio Renzi et al. "Bacterial canker on kiwifruit in Italy: anatomical changes in the wood and in the primary infection sites". In: Phytopathology 102.9 (2012), pp. 827-840.

[231] Mitja NP Remus-Emsermann et al. "Variation in local carrying capacity and the individual fate of bacterial colonizers in the phyllosphere". In: The ISME journal 6.4 (2012), pp. 756-765.

[232] RD O'brien and SE Lindow. "Effect of plant species and environmental conditions on epiphytic population sizes of Pseudomonas syringae and other bacteria." In: Phytopathology 79.5 (1989), pp. 619-627.

[233] Gwyn A Beattie and Steven E Lindow. "Bacterial colonization of leaves: a spectrum of strategies". In: phytopathology 89.5 (1999), pp. 353-359.

[234] Mark Wilson and Steven E Lindow. "Ecological similarity and coexistence of epiphytic ice-nucleating (Ice+) Pseudomonassyringae strains and a non-ice-nucleating (Ice-) biological control agent". In: Applied and Environmental Microbiology 60.9 (1994), pp. 3128-3137.

[235] Mark Wilson and Steven E Lindow. "Coexistence among epiphytic bacterial populations mediated through nutritional resource partitioning". In: Applied and Environmental Microbiology 60.12 (1994), pp. 4468-4477.

[236] DC Arny, SE Lindow, and CD Upper. "Frost sensitivity of Zea mays increased by application of Pseudomonas syringae". In: Nature (1976), pp. 282-284.

[237] LM Kozloff, MA Turner, and F Arellano. "Formation of bacterial membrane ice-nucleating lipoglycoprotein complexes." In: Journal of bacteriology 173.20 (1991), pp. $6528-6536$.

[238] RM Skirvin et al. "The use of genetically engineered bacteria to control frost on strawberries and potatoes. Whatever happened to all of that research?" In: Scientia horticulturae 84.1 (2000), pp. 179-189.

[239] SE Lindow, DC Arny, and CD Upper. "Biological control of frost injury: An isolate of Erwinia herbicola antagonistic to ice nucleation active bacteria." In: Phytopathology 73.8 (1983), pp. 1097-1102.

[240] Thomas Paternoster et al. "Selection of a biocontrol agent based on a potential mechanism of action: degradation of nicotinic acid, a growth factor essential for Erwinia amylovora". In: International Microbiology 13.4 (2011), p. 195.

[241] Mortimer P Starr and Manley Mandel. "THE NUTRITION OF PHYTOPATHOGENIC BACTERIA IV.: Minimal Nutritive Requirements of the Genus Erwinia”. In: Journal of Bacteriology 60.5 (1950), p. 669. 
[242] DR Walters, AC Newton, and GD Lyon. "Induced resistance: helping plants to help themselves". In: Biologist 52.1 (2005), pp. 28-33. URL:

http://helicon.vuw.ac.nz/login?url=http://search. ebscohost. com/login. aspx?direct=true\&db=aph\&AN=16997650\&site=ehost - live.

[243] Maryline Magnin-Robert et al. "Biological control of Botrytis cinerea by selected grapevine-associated bacteria and stimulation of chitinase and $\beta-1,3$ glucanase activities under field conditions". In: European journal of plant pathology 118.1 (2007), pp. 43-57.

[244] Annie Bézier, Bernard Lambert, and Fabienne Baillieul. "Study of defense-related gene expression in grapevine leaves and berries infected with Botrytis cinerea". In: European Journal of Plant Pathology 108.2 (2002), pp. 111-120.

[245] Aziz Aziz, Alain Heyraud, and Bernard Lambert. "Oligogalacturonide signal transduction, induction of defense-related responses and protection of grapevine against Botrytis cinerea". In: Planta 218.5 (2004), pp. 767-774.

[246] W James Gooderham and Robert EW Hancock. "Regulation of virulence and antibiotic resistance by two-component regulatory systems in Pseudomonas aeruginosa". In: FEMS microbiology reviews 33.2 (2009), pp. 279-294.

[247] Michael D Parkins, Howard Ceri, and Douglas G Storey. "Pseudomonas aeruginosa GacA, a factor in multihost virulence, is also essential for biofilm formation". In: Molecular microbiology 40.5 (2001), pp. 1215-1226.

[248] Sophie Zuber et al. "GacS sensor domains pertinent to the regulation of exoproduct formation and to the biocontrol potential of Pseudomonas fluorescens CHA0". In: Molecular plant-microbe interactions 16.7 (2003), pp. 634-644.

[249] Asita Chatterjee et al. "GacA, the response regulator of a two-component system, acts as a master regulator in Pseudomonas syringae pv. tomato DC3000 by controlling regulatory RNA, transcriptional activators, and alternate sigma factors". In: Molecular plant-microbe interactions 16.12 (2003), pp. 1106-1117.

[250] Susanne B von Bodman, W Dietz Bauer, and David L Coplin. "Quorum sensing in plant-pathogenic bacteria". In: Annual review of phytopathology 41.1 (2003), pp. $455-482$.

[251] Beatriz Quiñones, Glenn Dulla, and Steven E Lindow. "Quorum sensing regulates exopolysaccharide production, motility, and virulence in Pseudomonas syringae". In: Molecular Plant-Microbe Interactions 18.7 (2005), pp. 682-693.

[252] DI Rouse et al. "A model relating the probability of foliar disease incidence to the population frequencies of bacterial plant pathogens." In: Phytopathology 75 (1985).

[253] Helene Feil et al. "Comparison of the complete genome sequences of Pseudomonas syringae pv. syringae B728a and pv. tomato DC3000". In: Proceedings of the National Academy of Sciences of the United States of America 102.31 (2005), pp. 11064-11069. 
[254] Hitendra Kumar Patel et al. "The Kiwifruit Emerging Pathogen Pseudomonas syringae pv. actinidiae Does Not Produce AHLs but Possesses Three LuxR Solos". In: PLOS ONE 9.1 (2014), e87862.

[255] Sujatha Subramoni and Vittorio Venturi. "LuxR-family solos: bachelor sensors/regulators of signalling molecules". In: Microbiology 155.5 (2009), pp. $1377-1385$.

[256] Daniela Büttner and Sheng Yang He. "Type III protein secretion in plant pathogenic bacteria". In: Plant physiology 150.4 (2009), pp. 1656-1664.

[257] Xiang Yang Shi et al. "Characterization of regulatory pathways in Xylella fastidiosa: genes and phenotypes controlled by gacA". In: Applied and environmental microbiology 75.8 (2009), pp. 2275-2283.

[258] C Robin Buell et al. "The complete genome sequence of the Arabidopsis and tomato pathogen Pseudomonas syringae pv. tomato DC3000". In: Proceedings of the National Academy of Sciences 100.18 (2003), pp. 10181-10186.

[259] Vinita Joardar et al. "Whole-genome sequence analysis of Pseudomonas syringae pv. phaseolicola 1448A reveals divergence among pathovars in genes involved in virulence and transposition". In: Journal of bacteriology 187.18 (2005), pp. 6488-6498.

[260] Tanja Petnicki-Ocwieja, Karin van Dijk, and James R Alfano. "The hrpK operon of Pseudomonas syringae pv. tomato DC3000 encodes two proteins secreted by the type III (Hrp) protein secretion system: HopB1 and HrpK, a putative type III translocator". In: Journal of bacteriology 187.2 (2005), pp. 649-663.

[261] Yingxian Xiao et al. "Identification of a putative alternate sigma factor and characterization of a multicomponent regulatory cascade controlling the expression of Pseudomonas syringae pv. syringae Pss61 hrp and hrmA genes." In: Journal of bacteriology 176.4 (1994), pp. 1025-1036.

[262] Derrick E Fouts et al. "Genomewide identification of Pseudomonas syringae pv. tomato DC3000 promoters controlled by the HrpL alternative sigma factor". In: Proceedings of the National Academy of Sciences 99.4 (2002), pp. 2275-2280.

[263] Wensheng Wei et al. "The gene coding for the Hrp pilus structural protein is required for type III secretion of Hrp and Avr proteins in Pseudomonas syringae pv. tomato". In: Proceedings of the National Academy of Sciences 97.5 (2000), pp. 2247-2252.

[264] Hye-Sook Oh and Alan Collmer. "Basal resistance against bacteria in Nicotiana benthamiana leaves is accompanied by reduced vascular staining and suppressed by multiple Pseudomonas syringae type III secretion system effector proteins". In: The Plant Journal 44.2 (2005), pp. 348-359.

[265] John T Heap et al. "Integration of DNA into bacterial chromosomes from plasmids without a counter-selection marker". In: Nucleic acids research 40.8 (2012), e59-e59.

[266] Jac A Nickoloff. Electroporation protocols for microorganisms. Springer, 1995. 
[267] Herman P Spaink et al. "Promoters in the nodulation region of the Rhizobium leguminosarum Sym plasmid pRL1JI". In: Plant Molecular Biology 9.1 (1987), pp. 27-39.

[268] Martin Hahn and Torsten Stachelhaus. "Selective interaction between nonribosomal peptide synthetases is facilitated by short communication-mediating domains". In: Proceedings of the National Academy of Sciences of the United States of America 101.44 (2004), pp. 15585-15590.

[269] David F Ackerley and Iain L Lamont. "Characterization and Genetic Manipulation of Peptide Synthetases in Pseudomonas aeruginosa PAO1 in Order to Generate Novel Pyoverdines". In: Chemistry \& biology 11.7 (2004), pp. 971-980.

[270] Product testing report 17 November 2011. 2011. URL: http://www.kvh.org.nz/vdb/document/768.

[271] Michael Katz. "Anthelmintics". In: Drugs 13.2 (1977), pp. 124-136.

[272] SMF Calumpang et al. "Environmental impact of two molluscicides: Niclosamide and metaldehyde in a rice paddy ecosystem". In: Bulletin of environmental contamination and toxicology 55.4 (1995), pp. 494-501.

[273] Greens Warn Of Kiwifruit Spraying Dangers To Health. 2011. URL: http://www . greens.org.nz/press-releases/green-party-warns-dangershealth-and-agriculture-over-kiwifruit-spraying.

[274] Call to prosecute kiwifruit growers. 2012. URL: http://www.stuff.co.nz/nelsonmail/news/7008102/Call-to-prosecute-kiwifruit-growers. 